UCRL--53758

DE87 000716

\title{
Spectroscopic Analysis of Low Z Impurities in the Tandem Mirror Experiment-Upgrade
}

\author{
Tong Lai Yu \\ (Ph. D. Thesis)
}

Manuscript date: September 1986

\section{LAWRENCE LIVERMORE NATIONAL LABORATORY} University of California - Livermore, California - 94550 


\section{ABSTRACT}

Two absolutely calibrated EUV instruments have been used to study the impurity characteristics in the Tandem Mirror Experiment-Upgrade (TMX-U). One instrument is a normal incidence spectrograph that measures the time histories of several impurity emission lines(300-1800 A) in a single shot; radial scans can be obtained on a shot-by-shot basis. The other instrument is a monochromator that measures time-resolved radiai profiles of a given impurity emissicn line in a single shot.

The common intrinsic impurities measured in TMX-U are $\mathrm{C}, \mathrm{N}, \mathrm{O}$ arid $\mathrm{Ti}$. It has been shown that large fractions of the oxygen and nitrogen in the plasma are associated with the neutral beams while the main source of carbon is the plasma wall. In general, the concentration of each of the impuritties is low $(<1 \%)$, and the power radiated by them is less than $10 \mathrm{KW}$, which is a small portion of the total input power to the plasma. The concentrations of the impurities can be reduced substantially by glow discharge cleaning and titanium gettering. No significant accumulation of impurity ions in the thermal barrier region has been observed.

Neutral beams were employed to inject nitrogen into the central cell. From the decay time histories of the emissions of the beam-injected nitrogen ions, we estimated that the global confinement time of the nitrogen ions in the plasma is between 1 and 10 ms which is consistent with the value estimated from the nitrogen concentration in the plasma. A one dimensional transport code has been employed to study the radial transport of the impurity ions in the plasma.

Impurity-study experiments were also conducted in the TEXT tokamak using the same normal incidence spectrograph and a time-resolving grazing incidence spectrograph. The TEXT experiment and some results from TMX-U enabled us to study possible new $n_{e}$ and $T_{e}$ diagnostics using line ratios of impurity emissions. The results show that the resonance structure is important in the calculation of excitation rates for low-Z elements at low snergy levels. 


\section{ACKNOWLEDGMENTS}

This research is the result of the cooperation of many people; without the support of them, my effort in the past few years would be in vain. I was very fortunate to work under several advisers and had the chance to work together with so many bright people. First of all, the contributions of Professur H.W. Moos were invaluable. He patiently guided me to do research, to explore the scientific world, to report the new discoveries and finally to write this dissertation. His scientific knowledge contributed much towards understanding the data presented here.

Dr. S.L. Allen at Lawrence Livermore National Laboraiory (LLNL) offered an immense amount of heip with setting up the instruments, and conducting specific EUV experiments. He also contributed valuable discussions on the data and the resulting reports.

I have benefited very much by working with Professor M. Finkenthal of Racah Institute in Israel. His broad knowiedge in atomic physics and his efficiency in working in this area impressed me very much.

At the Johns Hopkins University(JHU), Ms. M. Neikirk kept coast-to-coast lines of communication. Mr. L.K. Huang was very helpful in calibrating the 1i24-channel spectrograph at the National Bureau Standards(NBS) and at JHU. The cooperation with Dr. B. Stratton, Mr. L.K. Huang and Mr. S. Lippman on the JHU/TEXT experiment was a pleasant experience; the discussions with them on the data were also valuable. Mr. W. Ruff offered a lot of technical help. Dr. E. Mcgie was very helpful in consulting me to modify the data-acquisition electronics for particular needs and to debug the circuits after long distance shippment. The support from the machine shop in modifying the EUV calibration equipment at JHU is also acknowledged.

At LLNL، Dr. 1.C. Simonen, the experimental group leader, and Dr. F.H. Coensgen, the project leader, were always in full support of this project. I daeply appreciate the significant contributions of the whole TMX-U experimental group 
to this effort.

At the Fusion Research Center of the University of Texas at Austin, Dr. W. Hodge coordinated the JHU/TEXT experiments. Dr. K. Gentle, who was the director of the TEXT group, contributed much to these experiments. The great effort payed by the whole TEXT group during the JHU/TEXT experiment are gratefully acknowledged.

At the beginning of the project, Dr. R. Zimmermann and Dr. R. Bell helped in the instrumental calibrations and in setting up the 1024-channel spectrograph at LUNL.

I also appreciate Dr. R.A. Hulse for helping me when I wrote the one dimensional transport code.

Finally, I would like to thank those who helped me with my English writing and escorted me into tre Lab during the holidays. 


\section{TABLE OF CONTENTS}

Page

Chapters

I. Introduction 1

1. Aspects of Controlled Fusion 1

2. Thermal Barrier Potential in TMX-U 4

3. The Role Played by Impurities in Fusion Devices 7

4. Outline of the Contents 8

II. Instrumentation and Experimental Details 10

1. Introduction 10

2. The Normal Incidence 1024-channel Time-resolving Spectrograph 10 2.1. Physical Features of the Spectrograph 10

2.2. Data Acquisition 13

2.3. Data Processing 13

3. The 22-channel Normal Incidence Monochrornator 18

4. The Locations of the Instruments 20

III. General Features of Impurities in TMX-U 23

1. Introduction 23

2. Impurity Concentrations and Radiated Power 24

3. Sources and Control of Impurities 28

4. Accumulation of Impurity lons in Thermal Barrier Region 40

5. Measurement of Kinetic Energy of Impurity lons by 44

Doppler Shift

6. Estimation of FLieling Rate from Ly or 49 
IV. Impurity Transport in TMX-U

1. Introduction 51

2. Global Confinement Time of Beam-injected lons in TMX-U

3. Radial Transport of Beam-injected and Wall-originated impurities

V. Development of Possible New $n_{e}$ and $T_{e}$ Diagnostics 74

1. Introduction 74

2. Experimental Evidence for The Effect of Resonance Structures 75 on Electron Excitation Rates for Si III

2.1 Experimental Data 76

2.2 Discussions of Results 78

3. $n_{e}$ Diagnostics Using Be I-like lons $\Delta n=0(2-2)$ Emissions 82

VI. Conclusions 93

Appendices

A. Details and Calibrations of the Normal Incidence Spectrograph 95

1. Spectrograph 95

2. Detector System and Data Acquisition 98

3. Intensity Calibration 102

a. Calibration at SURF II 102

b. Calibration Using EUV Calibration Equipment 107

B. Time Histories of Beam-injected Particles 112

C. One Dimensional Numerical Model for Radial Profiles of Impurity lons 117

$\begin{array}{ll}\text { References } & 124\end{array}$ 


\section{Table of Illustrations}

Page

Fig. I.1 Schematic diagram of tandem-mirror axial profiles.

Fig. I.2 Single-end tandem configuration of TMX-U.

Fig. Il.1 Schematic of the time-resolving 1024-channel normal incidence spectrograph.

Fig. II.2 Computer output of a raw spectrum arid the corresponding fitted spectrum.

Fig. II.3 The computer printout of the concentrations and associated radiated power estimated from the spectrum shown in Fig. il.2.

Fig. Il.4 The 0.4-m monochromator with 22-channel Spatial Imaging Detector System (SIDS).

Fig. II.5 The positions of the spectroscopic instruments on TMX-U.

Fig. III.1 Typical EUV spectrum of TMX-U central cell plasma obtained with the normal incidence spectrograph. 
Fig. III.2 Time histories of impurity ionization states showing that oxygen and nitrogen are associated with neutral beams whereas carbon is not.

Fig. III.3 The TMX-U vacuum vessel and glow-discharge cleaning system. $\quad 30$

Fig. Ill.4 Pressure-time profile during a TMX-U run.

Fig. III.5 Carbon concentration vs. shot number for a running day.

Fig. Ill.6 The brightness of carbon emissions as function of shot number for the various experimental sequences.

Fig. III.7 Carbon concentration vs. ICRF power showing that carbon concentration increases with the ICRF heating power.

Fig. III.8 Nitrogen impurity ions at the thermal barrier region did not increase during the formation of the barrier $(\sim 30 \mathrm{~ms})$.

Fig. Ill.9 Spectra showing the effect of Doppler broadening of NV $12391243 \AA$.

Fig. III.10 Kinetic energies of N V ions obtained from Doppler widths vs. time.

Fig. IV.1 Time histories of different ions emissions (time resolution $2 \mathrm{~ms}$ ) in CC during nitrogen injection.

Fig. IV.2 Time history of N $\vee 1239$ (time resolution $\sim 1.2 \mathrm{~ms}$ ) in $\mathrm{CC}$ during nitrogen injection. 
Fig. IV.3 Time histories of different ions emissions in east end plug.

Fig. IV.4 Time profiles of nitrogen ions obtained from analytic equations described in Appendix $\mathrm{C}$ with electron temperature $30 \mathrm{eV}$ and confinement time $10 \mathrm{~ms}$.

Fig. IV.5 Simular to Fig. IV.4 except that now confinement time 60 used is $1 \mathrm{~ms}$.

Fig. IV.6 Radial profiles of N III to N V ions.

Fig. IV.7 Similar to Fig. IV.6 except that now the profiles of O III to O VI emissions are shown.

Fig. IV.8 Similar to Fig. IV.6 \& 7 except that now the profiles of neutral deuterium and CIV emissions are shown.

Fig. IV.9 This figure shows nitrogen ions profiles which 65 correspond to the case of $\mathrm{N}$-beam injection shots.

Fig. IV.10 Comparison of experimental and simulated density profiles 69 for nitrogen.

Fig. IV.11 Similar to Fig. IV.10 except that this figure describes the 70 oxygen impurity.

Fig. IV.12 Similar to Fig. IV.10 \& 11 except that this figure describes the carbon impurity and no neutral beam source has been assumed. 
Fig. IV.13 Comparison of experimental and simulated density profiles of the nitrogen-beam injection shots.

Fig. V.1 The upper half of the figure (a) shows a portion of the Si III spectrum between 1290 and $1315 \mathrm{~A}$. The lower half (b) shows the "fitted spectrum" .

Fig. V.2 Comparison of theoretical and experimental values for the ratio $A_{3}$.

Fig. V.3 Characteristics of TEXT tokamak.

Fig. V.4 Electron density and temperature near the edge of the TEXT tokamak plasma.

Fig. V.5 a) Simplified Grotiar, diagram of the $n=2$ levels of Be l-like ions showing the transitions used in this work. b) Theoretical values of the ratio $\mathrm{R}$ from C III to Si XII at temperatures near their corresponding ionization equilibrium temperatures for four different densities.

Fig. V.6 Comparison of experimental values and theoretical computations using the R-matrix method for three ions.

Fig. A.1 Grating orientation of the spectrograph. 
Fig. A.2 Intensified photodiode array of the normal incidence spectrograph.

Fig. A.3 Data acquisition of the spectroscopic system.

Fig. A.4 Distribution of flux 238-Mev beam on detector.

Fig. A.5 Distribution of flux of $140-\mathrm{Mev}$ beam on detector.

Fig. A.6 Photometric calibrations from SURF II at NLS̀ (solid curve) 108 and from EUVCE (solid dots).

Fig. A.7 Calibration of the Normal Incidence Time-resolving Spectrograph(NTS) using the EUVCE. 


\section{Chapter I Introduction}

\section{I.1 Aspects of Controlled Fusion}

The goal of fusion energy research is to confine a high temperature plasma composed of light elements such as deuterium (D) and tritium (T) long enough that the energy released in exothermic nuclear fusion reactions exceeds the energy input to the system. There are two main paths of research to reach this goal, namely, inertial confinement fusion (ICF) and magnetic confinement fusion (MCF). In inertial confinement, a high power infrared or visible laser or particle beam is employed to implode a small pellet of solid DT. The compressed ion of the pellet results in fusing of the nuclei ${ }^{1,2}$. (Recently, a new scheme of fusion called magnetically insulated inertially confined fusion (MICF) which combined the merits of magnetic and inertial confinement has been studied ${ }^{3-5}$. In this approach, the plasma pressure is coifined by the inertia of a heavy metallic spherical shell while the self-generated mag.netic field by laser injection plays the role of thermal insulation.) In magnetic confinement, the most popular magnetic configurations are tokamaks and mirrors. In the tokamak magnetic configuration, the plasma is produced and confined in a tcioidal vacuum vessel. Tokamaks were first developed in the USSR during the 50 's and 60 ' $\mathrm{s} 6,7$. Tokamaks are now operating in U.S., Europe, Japan, and some third world countries such as China and South Korea. At piesent, tokamaks have been most successful in reaching the goals mentioned above; the tokamaks TFTR(U.S. ${ }^{8}$, JET (EURATOM) ${ }^{9}$, and JT-60(Japan) ${ }^{10}$ are expected to reach this goal within a few years; the T-15 tokamak of the Soviet Union will also operate with comparable parameters in the next few years ${ }^{11,12}$. Details of tokamak fusion research can be found in several excellent reviews 7, 13-17.

Mirror machines have several attractive features including easier construction and possible simplications because of the linearity of these devices. At present, they are behind the tokamaks in achieving the goal of breakeven (i.e. 
the condition where output energy from the fusion reaction is equal to injected power to the reactor).

Several excellent reviews of mirror fusion have been published ${ }^{18-23}$. In the early 1950 's , simple magnetic mirror machines were constructed which used an intense solenoidal shaped field with increasing fields at both ends to confine charged particles. The particles move in a helical path along the magnetic field line and are therby confined in the radial direction. Axial confinement is achieved when particles are reflected from the region of stronger magnetic fieid. However, it was found that the simple-mirror geometry was uristable. The concave magnetic field line produces an outward gravitational-like force which pushes the plasina from the center of the system to the walls ${ }^{1}$. In the 1960's and 1970's, the simple mirror was modified to a minimum-B configuration which had magnetic field lines with a convex curvature, increasing in all directions away from the center. In this configuration, the gravitation-like force is focused inward, producing a s able configuration. The minimum-B configuration stablizing effect was first reported by loffe in $1961^{24}$. l.ater experiments on Baselball $\mathrm{I}^{25}, 2 \times 11^{26}, 2 \mathrm{XIIB^{27 }}$, Phoenix ${ }^{28}$ and $\mathrm{PR}^{29,30}$ demonstrated the large improvement of the minimum-B over the simple mirror. However, the experiments also showed that this configuration would be a very inefficient design for a real fusion reaction plant; it could never generate thermonuclear power much greater than the injection power. Therefore, a new mirror confinement system was proposed in the late 1970's.

The tandem-mirror configuration suggested by Dimov et al. ${ }^{31}$ and by Fowler and Logan ${ }^{32}$ can in principle attain a $Q$ value (the ratio of the thermonuclear power to the injected power) much larger than 1. A tandem mirror is composed of two end plugs each similar to the 2XIIB minimum-B mirror connected by a solenoidal central cell. The positive electric potential developed in the end plugs together with the high magnetic-mirror ratio confine the central cell ions (Fig.l.1). In a tandem mirror reactor, most of the fusion power will be produced in the central cell. By increasing the volume of the central cell, one can increase the $Q$ value to a very high value. Experiments on Gamma $6^{33}$ first indicated the promise of the tandem-mirror geometry. Later, experiments on Tandem Mirror Experiment $(\mathrm{TMX})^{34}$, and Phaedrus ${ }^{35,36}$ also demonstrated the characteristics 


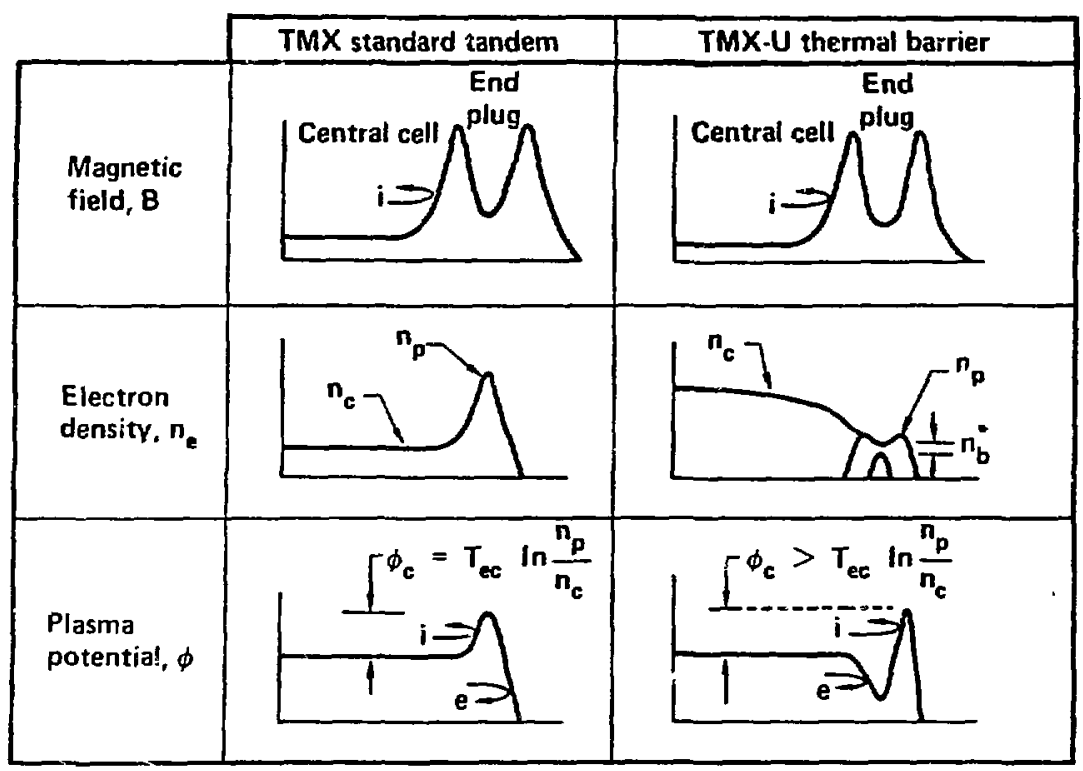

Fic. I.1 Schematic diagram of tandem-mirror axial profiles, compaing the "standard" tandem (left) with the TMX-U thermal barrier(right). The thermal barrier configuration allows an axial confining potential $\phi_{C}$ even if the central cell density, $n_{c}$ is greater than the plug density $n_{f}$. $n_{b}$ is the passing electron density which is the difference between ion and not-electron densities. 
of the tandem configuration. Though the experimental results of the tandem mirror machines were impressive, this tandem configuration is not suitable for a real fusion reactor. To confine the very energetic ions $(\sim 30 \mathrm{keV})$ in the central cell of a conventional tandem mirror reactor, very high-energy ( $500 \mathrm{keV}$ ) neutral beams would be needed to establish the required potential in the end plugs. This is beyond the scope of the present-day technology. The thermal barrier concept ${ }^{37}$ was introduced as a method for obtaining the necessary confining potertial without the high power and strong rnagnetic field requirements. TMX was redesigned to Tandem Mirror Experiment-Upgrade(TMX-U) to test the thermal barrier theory 38 .

\subsection{Thermal Barrier Potential in TMX-U}

The main goal of the TMX-U experiment is to demonstrate the leasibility of the formation of thermal barrier. As discussed above, Fig. I.1 compares both the standard tandem and thermal barrier tandem mirror configurations. In the TiMX (standard tandem) plasrnas the confining potential $\phi_{C}$ of a TMX plasma is described by the Boltzmann relation 1 .

An approximate analytic expression for the confinement time was first given by Pastukhov ${ }^{39}$ for a collisionless plasma (i.e. transit time between mirrors $\ll$ collision time) in a magnstic mirror with an ambipolar potential. The same expression was also derived by Chernin and Rosenbluth ${ }^{40}$ using a different approach. Because of the low ion temperature, the plasma ions in the TMX plasmis were not always completely collisionless and the Pastukhov expression was not always valid. However, computer modeling showed that the approprate confinement time expression for TMX was the sum of the Pastukhov time and the confinement time of a strongly collisional plasma 41 .

As shown in Fig.l.1, a thermal barrier is a region of negative potential between the central cell and the end-plug positive potential. Its main function is to isolate 
electrons in the end plugs from those in the central cell. The plug electrons can then be heated locally to a higher temperature and a large positive potential can be created even if the central cell density is larger than that of the end plug. In TMX-U, the barrier is formed by first injecting hot ions (actually energetic neutrals which ionize and charge-exchange to ions) in the end plugs. The ions are injected at $47^{\circ}$ to the magnetic field such that the ions will slosh back and forth within the end plugs, creating peaks in density (hence potential) at their turning points. The sioshing ion profile can evolve into a thermal barrier if the electrons at the trough of the sloshing ion profile are hot enough to be magnetically trapped for a long time. The required hot electron population for thermal barrier formation can be generated by heating the electrons using electron cyclotron resonance heating (ECRH). To maintain the thermal barrier, the energetic ions that become deeply trapped by the regative potential dip must be pumped away. This can be accomplished by charge-exchanging these ions with neutral beams located at each end of the machine.

Impurities might be scattered by the deuterium ions and tend to fill in the barrier. This increases the energy loss through impurity radiation, the loss rate of the sloshing ions, and the required pumping beam current. The theoretical basis of the thermal barrier can be found in references $37,42,43$.

The thermal barrier concept has been experimentally confirme 44.4 . The experiment used to demonstrate the thermal barrier was done with a single ended tandem configuration as shown in figure 1.2. In the experiment, electrons in one of the end plugs (west plug) were heated by both the first harmonic $\left(\omega_{\mathrm{ce}}\right)$ and second harmonic $\left(2 \omega_{\mathrm{ce}}\right) \mathrm{ECRH}$, and sloshing beams were injected into this plug. A thermal barrier was expected to form in this plug as shown in figure 1.2. The other end plug (east plug) was heated with second harmonic ECRH only and no sloshing beams were injected; no thermal barrier or plug potential were expected to form at this region.

The various potentials in this single-end tandem configuration were measured by three end loss analysers (ELA). As shown in figure I.2, the plug potential $\phi_{p}$ was measured by ELA3. The central cell potential $\phi_{e}$ was measured by ELA2. 

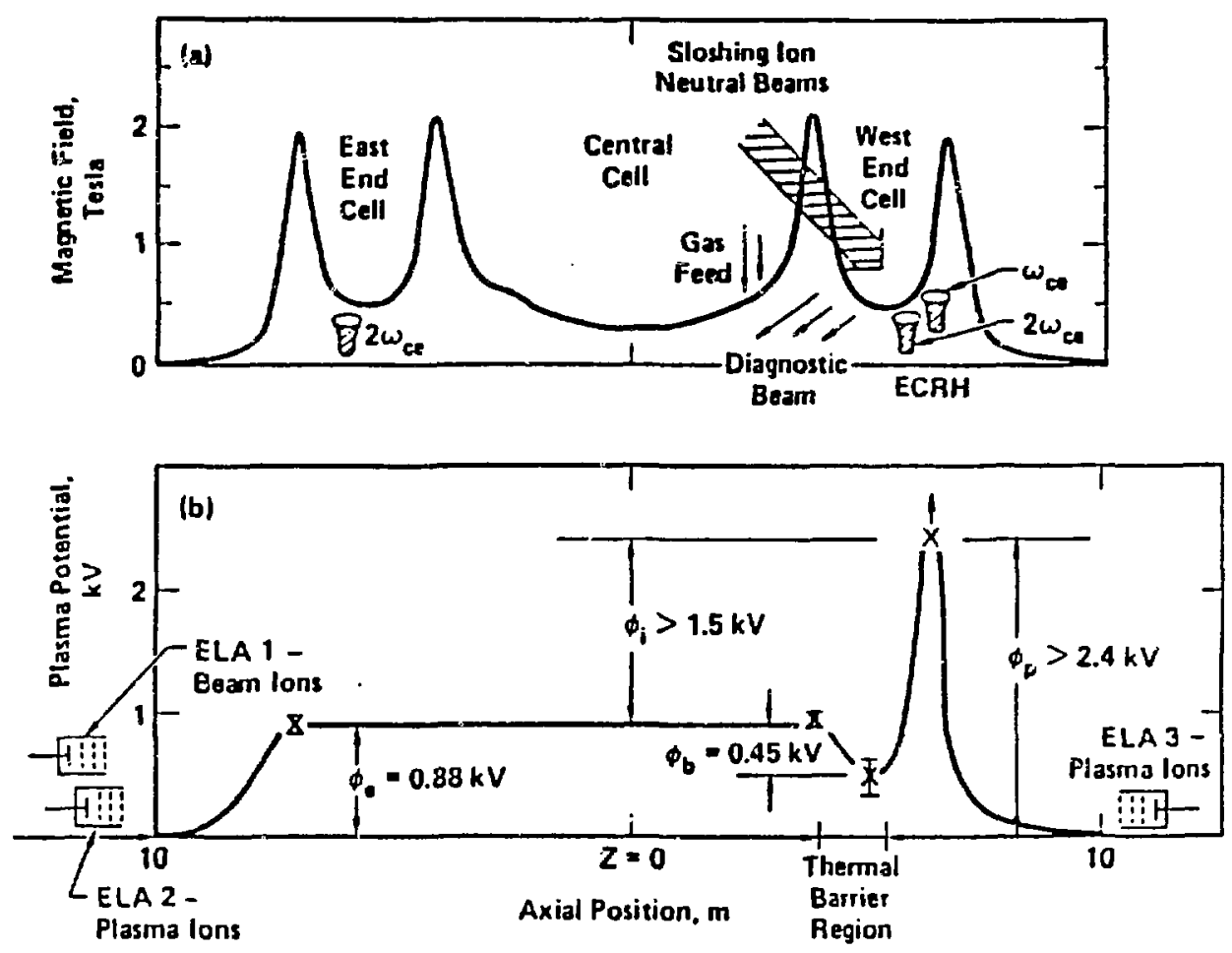

Fig. 1.2 Single-end tandem configuration of TMX-U (a) used for thermal barrier potential measurements. In (b) the measured potentials ( $X$ 's) are placed on the theoretically predicted axial variation of the plasma potential (solid line). (reference 44) 
ELA1 measured the potential dip $\phi_{b}$ by injecting a diagnostic neutral beam into the thermal barrier region of the west plug. The beam was injected at an angle such that the beam-ions created from charge-exchange with the plasma ions would not be trapped magnetically and would reach the end wall on the other side of the machine. Some beam-ions measured by ELA1 were created at the thermal barrier region. By analysing the energy spectra measured by ELA1, the value of the potential dip $\phi_{b}$ cuuld be deduced. The resulting measurements of the axial-potential profile is presented in figure I.2. A value of about $450 \mathrm{~V}$ of the potential dip $\phi_{b}$ was measured. This confirmed the existence of thermal barrier in TMX-U.

\subsection{The Role played by Impurities In Fusion Devices}

In a fusion device, impurities can be introduced into the plasma from machine walls, limiters, and auxiliary components such as neutral beams and radio frequency $\langle\mathrm{RF}$ heating systems. Light ions such as carbon and oxygen can be introduced from the walls 45 . Sputtering or high voltage arcs can release metallic atoms from internal structures such as limiters or RF antennas. It has also been observed that impurities such as oxygen and nitrogen are associated with neutral-beam-heating-systems. These impurities can cause significant power loss by radiation 45,46 . The presence of $3 \%$ of low-Z elements (oxygen), $1 \%$ of intermediate-Z elements (iron), or $0.1 \%$ of high- $Z$ elements (tungsten) can prevent the ignition of D-T plasmas 47 in a tokamak.

Fortunately, in tokamaks, impurity confinement times were found to be shorter than originally expected (see discussions in Chapter IV). However, as the confinement time of the plasma increases, the impurity confinement times may also increase. How the impurity confinement time scales with the machine size is still an unknown. In a tandem mirror device, the parallel confinement time 
of the impurities increases much faster than that of the plasma ions. This is because a multiply charged impurity ion sees a much larger end plug potential than a singly charged plasma ion. Moreover, the impurities can fill in the thermal barriers of a mirror machine and thus largely reduce the confinement of the system.

However, the existence of impurities in a fusion device is not always bad. For example, the energy confinement in a plasma containing a small amount of impurities may be better than the confinement in a clean plasma at the same density. We can also make use of the radiating property of the impurities to dump excess power to the edge of a plasma by creating a low-Z impurity halo surrounding the hot interior plasma; this can avoid local heat deposition on walls or limiters. Beam-injected ight impurities such as nitrogen can be used to heat the plasma. Since the rate of energy transfer from an ion to electrons is proportional to $Z^{2}$ of the ion, (where $Z$ is the excess positive charge of the ion), it is much more effective to heat the electrons with $N^{+5}$ than with $D^{+}$as long as the $\mathrm{T}_{\mathrm{e}}$ is high enough (>30 eV) that the radiated power from the injected low- $Z$ impurity ions is small.

The main purpose of this research is to investigate the effects of impurities on plasmas in the TMX-U device and to examine how we can make use of the special features of impurities to facilitate the study of the fusion plasma.

\section{I.3 Outline of the Contents}

This report consists of six chapters and three appendices. Chapter lis this Introductory chapter.

Chapter II presents the spectroscopic instruments used in this project. The details of the instruments and the calibration of the principal instrument are 
described in Appendix A which complements chapter H. Chapter II also describes in detail the data processing of the spectroscopic system.

Chapter III describes how intrinsic impurities behave under different machine conditions. In chapter III, we estimate the concentrations of intrinsic impurities in the TMX-U plasma, the resulting radiated power, and how these are influenced by glow discharge cleaning and titanium gettering. We also discuss the filling of the thermal barrier by impurites, the estimation of impurity ion energies froin Doppler broadening and the calculation of fueling rate from Lyo brightness.

Chapter IV describes the transport of impurities in TMX-U plasmas. Neutral beams were used to inject energetic nitrogen into the plasma. Models have been developed to model the behravior of the beam-injectud impurities. Appendix $B$ and $C$ describe in detail the models discussed in chapter IV.

Chapter $V$ gives a description on the developmerit of possible new $n_{\theta}$ and $T_{e}$ diagnostics using line ratios of impurity emissions. In that chapter, we also describe an importart, new measurement relevant to the application of such : diagnostic method which snows that the resonance structures are important in the calculation of excitation rates for low- $Z$ elements at low energy levels.

Chapter VI is the conclusions; the important results are summarized in this chapter. 


\section{Chapter II Instrumentation and Experimental Details}

\section{II.1 Introduction}

Two EUV instruments are employed to measure the impurity behavior in the central cell and the end plugs of TMX-U. The principal instrument used for studying EUV emissions is a 0.4-m normal incidence 1024-channel time-resolving spectrograph which was built at The Johns Hopkins University (JHU). A secono instrument was a 0.4-m normal incidence monochromator equipped with a spatial imaging detector acquired by Lawrence Livermore National Laboratory (LLNL) for the TMX experiment. The results from a third grazing incidence spectrograph located in the central cell are presented elsewhere 48,49 .

Details of the design and construction of the two normal incidence EUV instruments have been described in detail elsewhere ${ }^{50-52}$. Here, we only describe briefly the operating mechanisms of the two instruments. Because the spectrograph is the major instrument used in the impurity study in TMX-U, we describe it and its calibration in more detail in Appendix A

\section{II.2 The Normal Incidence 1024-channel Time-resolving Spectrograph}

\section{II.2.1 Physical Features of the Spectrograph}

An outline drawing of the normal incidence spectrograph is shown in figure $11.1^{50}$. The spectrograph is a $0.4-\mathrm{m}$ normal incidence $f / 30$ system with seven gratings. Its seven selectable gratings permit the instrument to be 


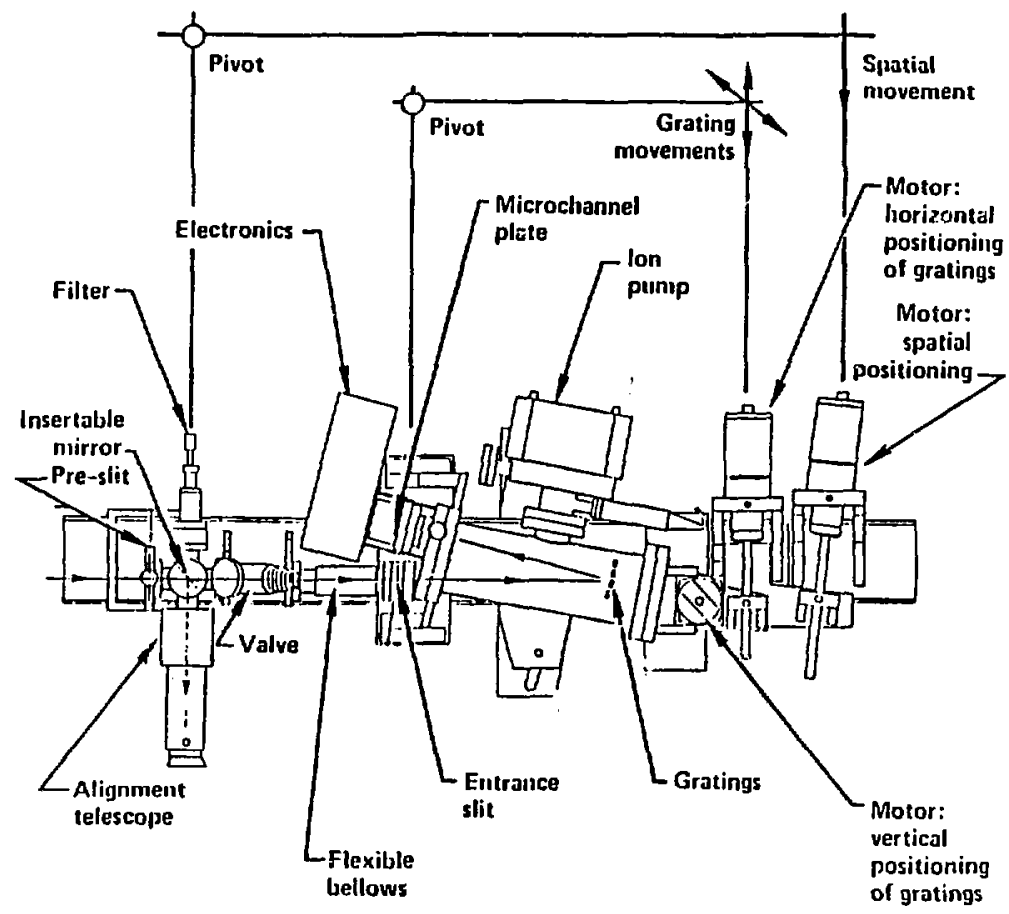

$140 \mathrm{~cm} \longrightarrow$

Fig. Il.1 Schematic of the time-resolving 1024-channel normal incidence spectrograph 50 . 
operated in a survey-resolution(5- $\AA$ ) mode or in one of several high-resolution ( $1 \AA$ ) modes covering smaller wavelength ranges. In the survey mode, emissions from 300 to $1800 \AA$ can be measured simultaneously. The detector system of the spectrograph consists of a microchannel plate image intensifier with a Cul photocathode fiber-optically coupled to a 1024-channel photodiode array. The output of the photodiode array is scanned and digitized by an electronic controller. The digitizer used is a 12-bit A/D converter.

The data are stored in a local CAMAC memory and are read and processed by a local desktop computer (HP 9836) or the TMX-U data-acquisition system. After being read by the local computer, the data is stored in floppy disks. The shortest readout time of the 1024-chanilel data set (or frame) is about $4 \mathrm{~ms}$; a channel selector can read out channel subsets with a minimum time resolution of $1 \mathrm{~ms}$. We can also increase the signal to noise ratio (at the expense of the time resolution) by increasing the readout time of the array to a maximum of $60 \mathrm{~ms}$. This is particular useful in the intensity calibration of the instrument. The instrument has several entrance slits with different sizes; at the expense of signal strength, higher spectral resolution can be obtained with a narrower slit. The instrument also has two preslits with different sizes; higher radial resolution ( $\sim 4 \mathrm{~cm}$ ) can be obtained with a smaller preslit. The detector system can be operated at five different voltages; the sensitivity of the system varies by about a factor of 100 over this voltage range. This enables the instrument to be operated over a very large dynamic range. The spectrograph can be scanned radially by remote control to view different positions of the plasma on a shot-by-shot basis.

We have calibrated the the instrument in absolute intensity by using the SURF II facility of the National Bureau of Standards (NBS) and also by comparison with NBS-reference-photodiodes at The Johns Hopkins University (JHU). The wavelength calibration was done using a Penning discharge lamp 53 and the spectral lines produced in tokamaks and mirror discharges. More detail discussions of this instrument and the calibration are presented in Appendix A. 


\section{Il.2.2 Data Acquisition}

Computer software development plays a more important role than ever before in the development of a spectroscopic diagnostic system, because of the large amount of data provided by one and two dimensional detector anay. The 1024-channel spectrograph acquires all of the major plasma emissions at a 1-4 ms rate in a $100 \mathrm{~ms}$ plasma shot. Withou: suitable software, we cannot handle the data efficiently and deduce useful physical quantities between shots. Specifically, in our case, we are interested in the concentrations of the impurities and the associated radiated power in each shot and the use of this data to obtain trends in the impurity data.

The spectroscopic system is controlled by the HP 9836 microcomputer and the data is acquired through a CAMAC crate controller. After the controller has been cleared by the computer, the two dual port memories of the CAMAC system will wait for data from the spectrograph. When a plasma shot comes, a TTL (transistor-transistor logic) pulse generated by the TMX-U control system triggers the electronics of the spectrograph to scan the Reticon array and to output the data to the CAMAC memories. The CAMAC memories will receive data until filled. The CAMAC crate controller then issues a signal to inform the computer that data is available. The computer then reads in all the data stored in the dual port memories. The relevant data is then stored in a floppy disk and processed. Meanwhile, a timer resets the electronics to wait for another shot.

\section{II.2.3 Data Processing}

The data reduction is also done using the same HP 9836 which controls the spectrograph system. To process the data, the computer program first locates all the frames ( $a$ frame is the 1024 channel data set; the spectrograph electronics output a sync word at the start of each frame). If several pixel groups have been selected, they have to be located also. After locating all the 
frames, the data is theri processed. The computer plots out the time histories of several interesting impurity lines on both the CRT screen and a dot-matrix thermal printer. The time the shot occured is also printed out to keep track of the shot sequence. This is followed by the plotting of a sequence of frames so that we can see the time evolution of all the features in the recorded spectra.

The next task the software does is to locate the frame with the maximum signal and search for all the impurity lines in that frame. This search is performed by first smoothing the spectrum by averaging the counts of a pixel with that of its neighbouring pixels. Next, the value of the counts of the pixel is compared with that of the nearby pixels; by this method, the peaks of all the lines in the spectrum can be located. The wavelengths of the located lines are obtained from the wavelength calibration which has been included in the software. The wavelength of each of the lines is then compared with those from a table of ionic species which are mostly likely to appear in the plasma. When the wavelengths match, the line is identified. The computer will plot out the spectrum in a larger scale with the identified lines labeled with their corresponding symbols. By looking at this labeled spectrum, we can immediately have an idea of what kind of impurities exist in the associated plasma shots and know roughly how they compare to those of the previous shots.

Because the positions of the lines are known, the program next calculates the total counts(area) of each line by performing a simple nonlinear least square fit to the data points of each of the lines. For simplicity, we have chosen the fitting function $f(x)$ to be a Lorentzian superimposed on a background level. That is,

$$
f(x) \sim C_{0}+C_{1} /\left(1+x^{2} / \delta^{2}\right)
$$

where $\mathrm{C}_{0}, \mathrm{C}_{1}$, and $\delta$ are the fitting variables. The area of the line is given by

$$
A=C_{1} \pi \delta
$$


After the fitting variables are found, all the located lines can be reconstructed using equation (2.1) to produce a "fitted spectrum". The computer also plots out the fitted spectrum which is to be compared with the raw spectrum (Fig. II.2); this will give us a rough idea how yood the fit is. The area of a line can also be estimated by directly summing up the counts of the pixels covered by the line and subtracting an appropriate background. However, the error arising from the direct summing method can be large if the line is weak as in the case of Fig. II.2; moreover, it is difficult to handle the "tail" of the line. Nevertheless, the program does use this method to obtain values of the aieas of the lines for comparison or reference.

Now, the corresponding brightness of a line can easily be calculated as it is proportional to the quotient of the area of the line divided by the efficiency at that pixel position; the efficiency calibration has also been stored in the program. (Actually, the number of efficiency values at various positions that have been stored is only about thirty. Other values are obtained by interpolation by a spline routine.)

After the brightness has been found, the density (hence concentration) of each of the ionic species and the associated radiated power can be estimated using the constant density model discussed in Chapter III. Values of excitation rates are required in the calculation of the densities. Therefore, the program can only calculate the densities of the ions whose emission lines have been identified earlier. The value of the line density of the plasma is required in the calculation. The program either assumes a certain reasonable value in the calculation or reads in the value from the main TMX-U data acquisition computer (HP 1000) through the Hewlett Packard Shared Resource Management (SRM) computer network. (After this print out, the computer resets the CAMAC to wait for another shot.) All these are printed out in taibular form. Figure 1:.3 shows a typical print out of the final results. If necessary, the processed data (e.g. brightnesses of the lines) can be sent to the main computer (HP 1000) through the SRM network; the EUV data is then stored in the TMX-U data base. The program takes about three minutes to do all the data processing for each shot and the processing is done between shots. 

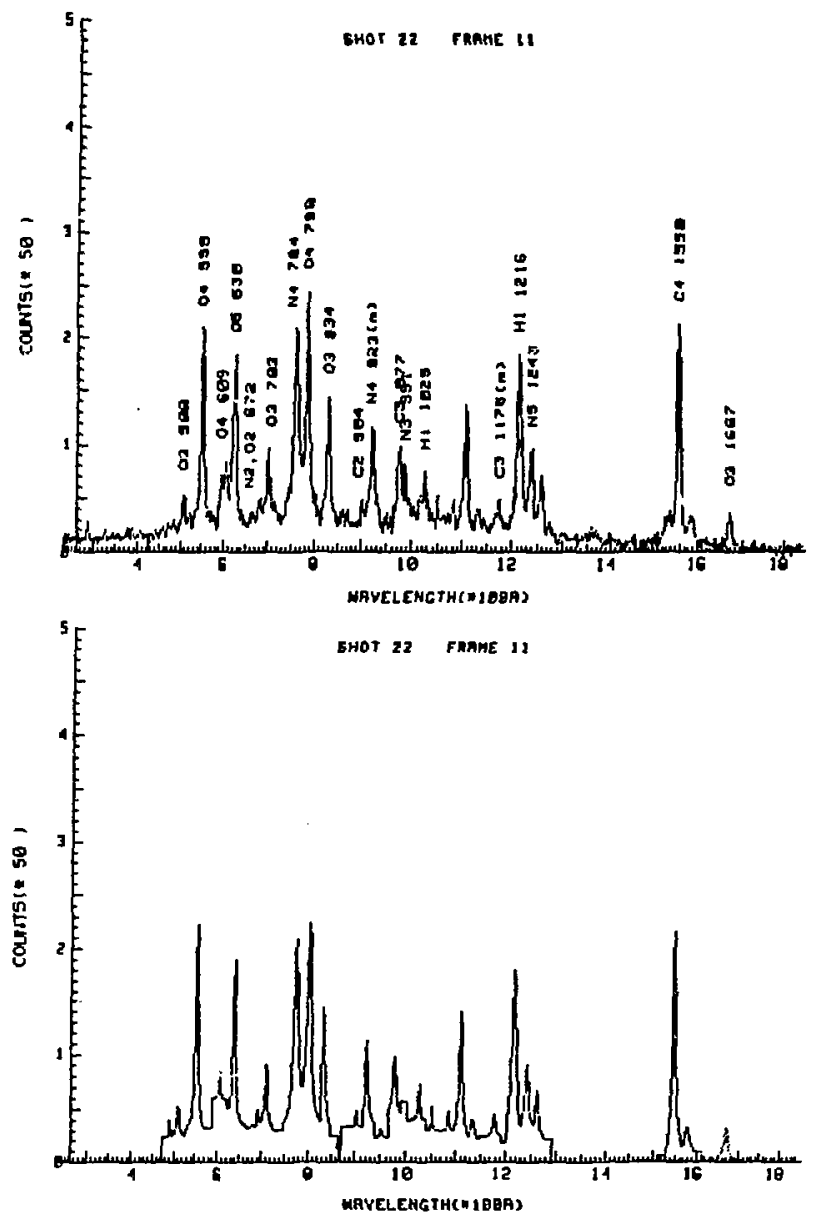

Fig. II.2 A computer output of a raw spectrum and the corresponding fitted spectrum below; the signal in this spectrum was very weak and therefore appeared "noisy". 


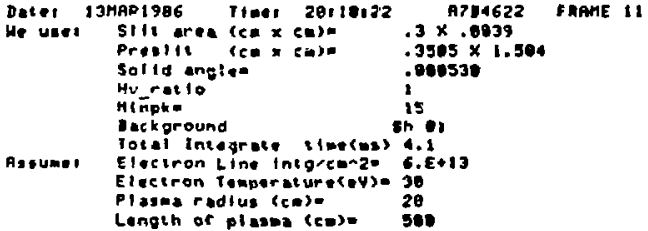

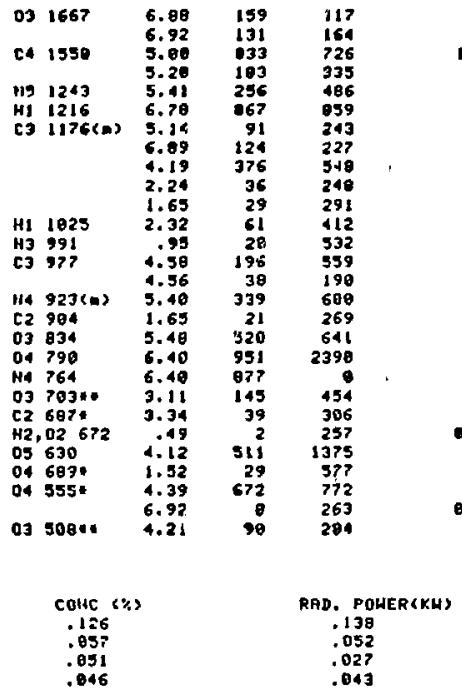

TOTAL FONER BY COHSIDERIIIG ALL FITTED LIHES: *3 KH

TOTAL FOHER DY COHSIDERIHG ALL THE PIKELS: .7 KH

Fig. Il.3 The computer printout of the concentrations and associated radiated power estimated from the spectrum shown in Fig. II.2. SIG is the width 8 of a line discussed in the text; INTG is the area of the line obtained by the fiting while DIR_SUM is the area obtained by directly summing up the pixels, counts of the line; BRIG and DEN are the brightness and density of the line respectively. 
Because the software can estimate the concentration and the associated radiated power for each species in the plasma between shots, the trend of the impurity characteristics can be monitored easily; this is particularly useful in monitoring spurious impurity effects in the plasma and real-time changes such as a vacuum leak. As a good example, we have monitored the change in the impurity characteristics in the plasma under different machine conditions (see figure III.6 in next chapter) which enables us to draw significant conclusions about the impact of impurities in the startup of a plasma as discussed in Chapter III.

\section{II.3 The 22-channel Normal Incidence Monochromator}

The design and construction of the spatially imaging monochromator, shown in figure 11.4 , has been described in detail in reference 51 . Briefly, the instrument is a $0.4-\mathrm{m}$ normal incidence monochromator with 22 channels. It is capable of measuring time-resolved spatial profiles of impurity emissions by using the entrance slit as a one-dimensional analog of a pinhole camera. Each position in the plasma corresponds to a particular point on the detector. We can select the desired wavelength precision rotations of the grating using a remote controller. The detector has 22 discrete anodes coupled to a pair of microchannel plates. The two sets of the photo-channels of the two plates form a chevron configuration. Each of the 22 anodes maps to a certain region of the plasma. The charge from the anode is measured by a pulse amplifier-discriminator. The digital data is then stored in a local CAMAC memory which can be read by a local desktop computer or by the main TMX-U data acquisition system. The time resolution of the instrument varies between 0.1 and $1 \mathrm{~ms}$. This instrument is also absolutely calibrated both in wavelength (the tilting angle of the grating) and intensity. The intensity calibration was done by comparison with photodiodes which had been calibrated at NBS. The details of the calibration can be found in reference 52 . 


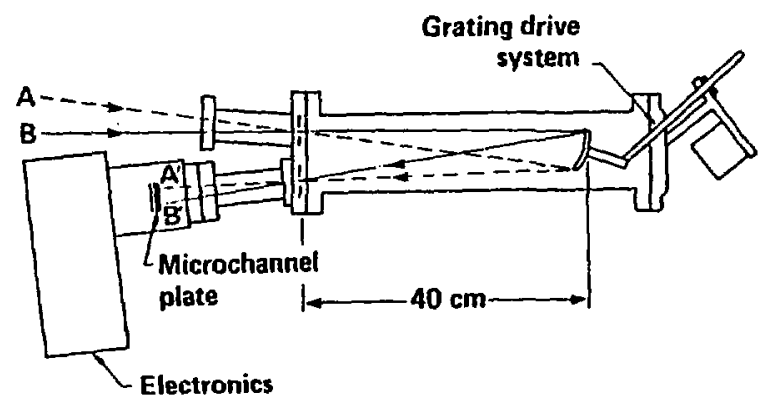

Fig. II.4 The 0.4-m monochiomator with 22-channel Spatial Imaging Detector System (SIDS). The two tays A and Btrom the plasma are imaged at the detector to the two points $A^{\prime}$ and $B^{\prime}$, respectively. The instrument has its own separate high-vacuum sysiem and is absolutely calibrated in wavelength and intensity. 


\section{II.4 Locations of the Instruments}

The locations of the instruments in TMX-U are shown in figure II.5. Throughout this project, the main emphasis was placed upon studying the central cell of TMX-U; the 1024-channel spectrograph which was the principal EUV instrument in this study was mounted on the central cell for most of the time and obtained an extensive amount of data from this region. A limited anount of data was obtained with the 1024-channel mounted on the west end plug of TMX-U. For the data presented here, the 22-channel monochromator was always mounted on the east end plug.

We first calibrated the 1024-channel spectrograph at the NBS in March, 1982. In April and May, 1982, we calibrated it at JHU using the extreme ultraviolet calibration equipment which was designed and b:silt by the $\mathrm{JHU}$ plasma spectrograph group ${ }^{54}$. The spectrograph was then shipped to LLNL at the end of June, 1982 and mounted on the central cell(CC) at the same time. The first set of data was taken in August, 8, 1982. The instrument stayed at LLNL until May 1984. During this period, it viewed the CC most of the time and was mounted on the west end plug for about two months.

In May, 1984, we shipped the instrument back to $\mathrm{JHU}$ and calibrated it at NBS. We found that the sensitivity of the instrument was unchanged. The instrument was taken back to LLNL in June, 1984, and was mounted on the CC of TMX-U. (During this time, the instrument was brought up to atmospheric pressure for a couple of days by accident. However, later calibrations show that this event has not changed the sensitivity of the spectrograph.)

At the end of August, 1984, we shipped the instrument to University of Texas at Austin and mounted it on the TEXT tokamak. We performed impurity study experiments on TEXT for about three months. The data obtained from the 1024-channel spectrograph on TEXT enable us to develop possible new $T_{e}$ and $\mathrm{n}_{\mathbf{e}}$ diagnostic methods using impurity emission line ratios (see Chapter $V$ ). This diagnostic method will be very useful in the future TMX-U or MFTF operation. 


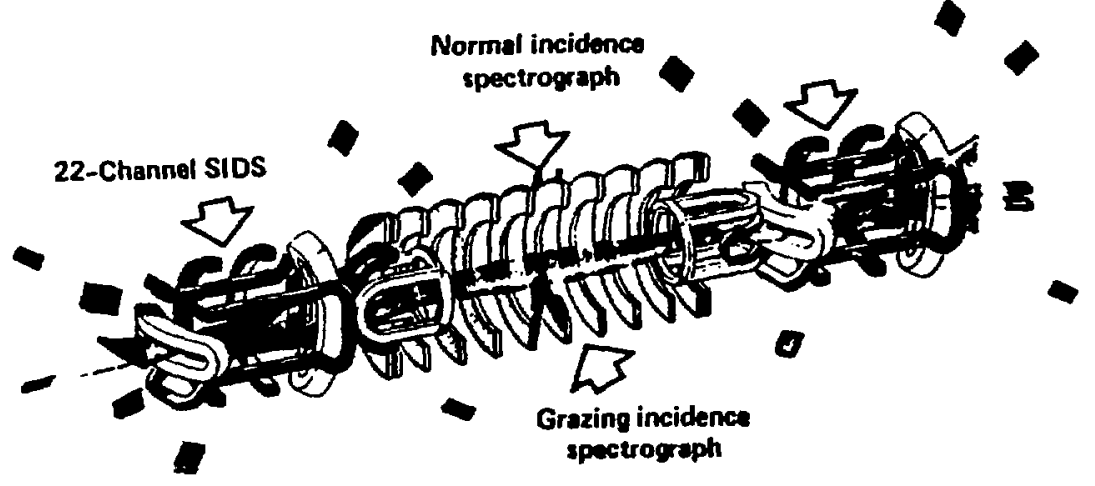

Fig. 11.5 The positions of the spectroscopic instruments on TMX-U. Because of its size, the Grazing incidence Spectrograph is located on the side of the central cell and views the plasma along a horizontal chord. The other two instruments can belocated either in the end cell $\alpha$ in the central cell (at the locations of the arrows on the top of the machine). Their positions can be changed while TMX-U is under vacuum. 
In January, 1985, the instrument was shipped to LLNL and mounted on the CC of TMX-U. In June of that year, we took it back to JHU. We calibrated it again using both the EUV calibration equipment at JHU and the SURF II at NBS. In September of the same year, it was returned to TMX-U and was mounted on the central cell and took data from October, 1985 to March, 1986. 


\section{Chapter III General Features of Impurities in TMX-U}

\section{III.1 Introduction}

TMX-U is the largest mirror device ever operated at LLNL. Previous impurity studies on the single mirror machine 2XIIB indicated that the neutral beams were a source of oxygen impurities 55,56 . These experiments were followed by the Tandem. Mirror Experiment (TMX). Again, the major impurity found was oxygen, and the impurity characteristics of the TMX end plugs were similar to the $2 X \| B$ results 57 . Lesser amounts of nitrogen, carbon and titanium were also observed ${ }^{58}$. Radial penetration of cold impurity ions into the central cell plasma of $T M X$ was very inefficient ${ }^{59}$. As mentioned in Chapter 1 , the present TMX-U device is an improved tandem mirror design which has demonstrated enhanced axial confinement with a thermal barrier 37 . The thermal barrier potential profile is a result of a hot electron population formed by ECRH heating, and a sloshing ion population formed by neutral beam injection; the potential region must be assured that it would not be filled by plasma ions or impurity ions. Central cell ions and electrons are heated by neutral beam injection and lon-Cyclotron Resonant Heating(ICRH).

Because of the addition of more sophisticated EUV instruments that obtain time-resolved spectra, the impurity measurements on TMX-U have been extended from those on $2 X I I B$ and TMX to include measurement of impurity concentrations in a single shot. These measurements also provided identification of impurity sources, time-resolved measurement of Doppler shift of emission lines, and estimation of fueling rate from L $y_{\alpha}$ emissions. The 22-channel monochromator monitored the variation of the impurity densities at the east end plug. We found that the impurity results from TMX-U are similar to those of TMX; the impurity concentrations continue to be low; the power radiated by the impurities is only a small fraction of the input power. The data obtained at the end plug shows that there is no significant accumulation 
of impurity ions in the thermal barrier region. Glow-discharge cleaning and titanium gettering of the machine walls have been shown to reduce the impurity concentrations in the plasma.

\section{III.2 Impurity Concentrations and Radiated Power}

Under normal conditions, we found that the most abundant impurities in TMX' $U$ were carbon, nitrogen, oxygen, and smaller amounts of titanium. Silicon was observed under unusual operating conditions. Figure III. 1 shows a typical spectrum used to estimate the concentrations and radiated power of the impurities as discussed below. The measured brightness of a line emitted from an impurity ion is proportional to the integral of the product of the excitation rate, the electron density and the impurity ion density integrated along the line of sight of the instrument. The 1024-channel spectrograph measures the average brightness of one radial chord; only limited shot-by-shot scans were taken to obtain typical profiles. Therefore, we employ a model for the radial profiles 55,56 that allows us to calculate the impurity density from the chord-averaged brightness. Other models have been used to estimate the density 57 and the results show that they are relatively insensitive (within a factor of two) to reasonable choices of the profile. In our model, we assume that the ion density is a constant in radius and is zero when the radius is larger than a certain value. With this assumption, upon measuring the electron density 60 and temperature 61 , we are able to calculate the value of the impurity ion density of the corresponding ionization state ${ }^{52,62}$. The total density of an impurity species in the plasma is the sum of the densities of the different observed ionization states (including the metastable states). The details of the calculations performed can be found in reference 48 .

Using the constant-ion-density model for the radial profile of the emissions, we can easily calculate the radiated power associated with each emission line from the measured brightness. Because most of the significant emissions for 


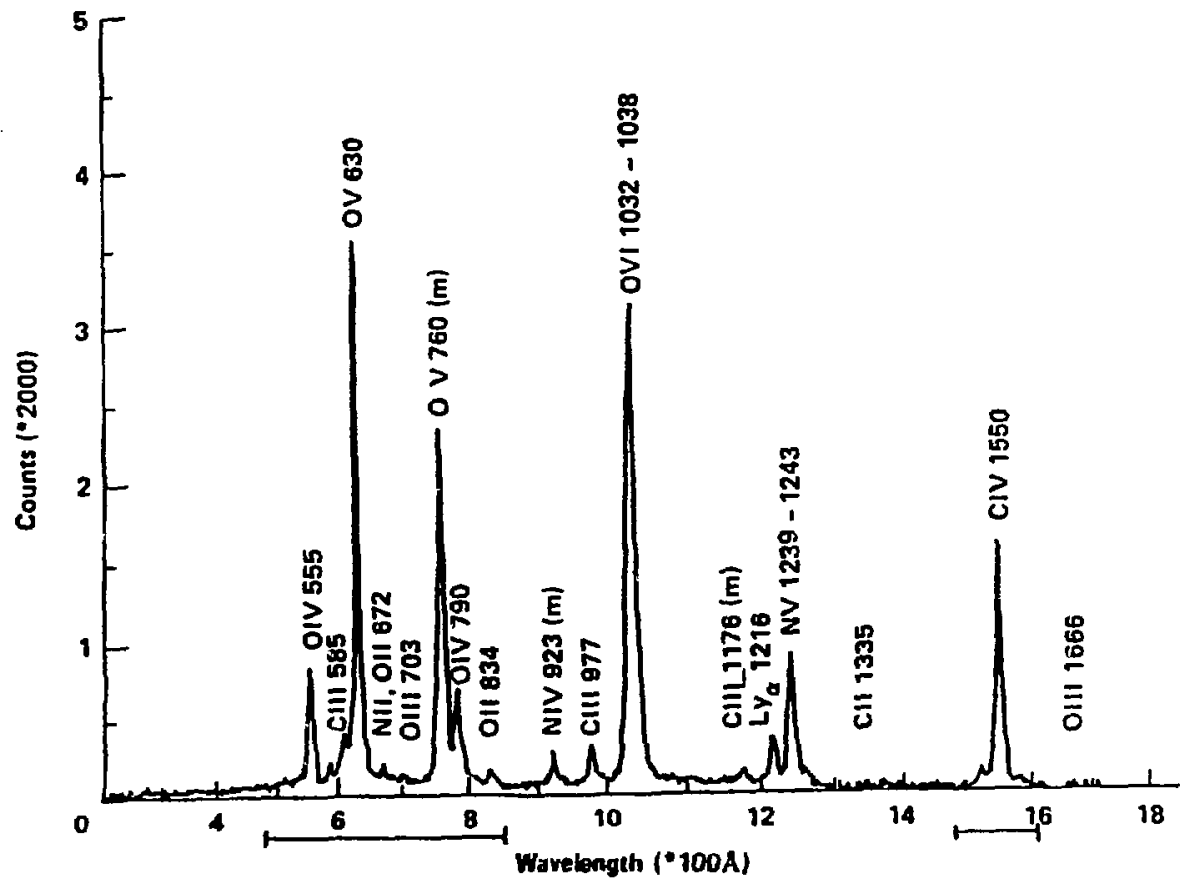

Flg. II.1 Typical EUV spectrum of TMX-U central cell plasma obtained with the normal incidence spectrograph. The solid bars incicate how separate spectral regions can be selected and measured with higher time resolution. One to seven spectral regions can be selected at a time. 
each impurity species are in the wavelength range of the instrument, the power associated with each ionization state can be summed to obtain the total for each species. Note that because we calculate the radiated power directly from the brightness, the total power can be determined even if the source of particular emission features is not known 63 .

In the central cell, during sloshing-ion-density operation for an axial electron density of $5 \times 10^{12} \mathrm{~cm}^{-3}$, each of the $\mathrm{C}, \mathrm{N}$ and $\mathrm{O}$ impurities had a low concentration $(<1 \%)^{64}$. The total power radiated by the impurities was about 3-10 KW, which is a small fraction of the total input power to the system(typically several hundred KW). The central cell impurity concentrations and associated radiated power for a typical plasma shot of the TMX-U central cell are presented in Table III.1. Note that in Table III.1, we have only included the measured impurity states from the1024-channel spectrograph, that is the ionization states up to C IV, NV and O VI. Emissions from the next ionization states (He-like) C V, N VI, and O VII are outside the wavelength range of this instrument. (Rough estimates of the concentrations of these ions based on the measured line ratios show that each of these ionization states has a concentration less than $1 \%$.) While some of the impurities may be in these states, they do not add a significant amount of radiated power. This is because the excitation rates of the He-like ions emissions are very small at the current temperature and thus the emissions are very weak. During the thermal-barrier mode which is operated ai a lower density $\left(\sim 1 \times 10^{12} \mathrm{~cm}^{-3}\right)$, the radiated power is usually less than $2 \mathrm{KW}$. The impurity concentrations in the end plugs are in general higher but still comparable (factor of 2) to those in the central cell. 
Table III.1 Impurities calculated " for a plasma shot (at a central density of $4 \times 10^{12} \mathrm{~cm}^{-3}$ ) in the TMX-U central cell.

Impurity Concentration(\%) Radiated Power(KW)

$\begin{array}{lll}\text { Carbon } & 0.06 & 0.3\end{array}$

$\begin{array}{lll}\text { Nitrogen } & 0.17 & 0.7\end{array}$

$\begin{array}{lll}\text { Oxygen } & 0.82 & 2.7\end{array}$

"Amounts include only the impurity states measured by the 1024-channel normal incidence spectrograph, that is, the ionization states up to $\mathrm{CN}, \mathrm{NV}$, and $\mathrm{O} \mathrm{VI}$. 


\section{III.3 Sources and Control of Impurities}

The measurement of the time-resolved EUV spectra in a single shot also facilitates the identification of the impurity sources. In some of the experiments, the central cell neutral beams were turned off during the steady state portion of the plasma shot. As shown in figure III.2, when the neutral beams were turned off, the oxygen and nitrogen impurity emissions decreased significantly while the carbon line intensities remained relatively unchanged. We conclude from this data that the oxygen and nitrogen emissions are associated with the neutral beams. Previous results showed that $t \%$ of the neutral beam current is composed of oxygen 55,56 . We also observed from the Doppler broadening of nitrogen emissions that the nitrogen ions are energetic (see next subsection). Further evidence that neutral bearns are the source of these impurities is that their emissions are not reduced by glow-discharge cleaning and titanium gettering to as great an extent as are carbon emissions.

The impurities associated with the neutral beams can be controlled by titanium-gettering the arc chambers of the neutral beams. It has been shown that the oxygen impurities in a neutral beam can be reduced by at least a factor of eight 65 by this technique. Titanium getters have been installad in the TMX-U neuiral-beam sources, but have never been used during the plasrna shots. This is because under normal conditions, the impurity emissions are quite low and do not have serious effects on the plasma ${ }^{66}$.

The vacuum chamber wall is an important source of carbon in TMX-U. As shown in Fig. III.3, the vacuum vessel consists of a room-temperature wall surrounded by two liners which are cooled by liquid nitrogen 67 . Some hydrocarbons are only loosely bound to the wall and may be released and subsequently enter the plasma when bombarded by particles during a plasma shot. Data from a residual gas analyser show that the main background gas in the machine is methane and some other hydrocarbons. Figure III.4 shows the pressure-time profile during a TMX-U run. The pressure due to methano increases when the two liners are no longer filled with liquid nitrogen. These data indicate that the walls can be a source of the observed carbon 


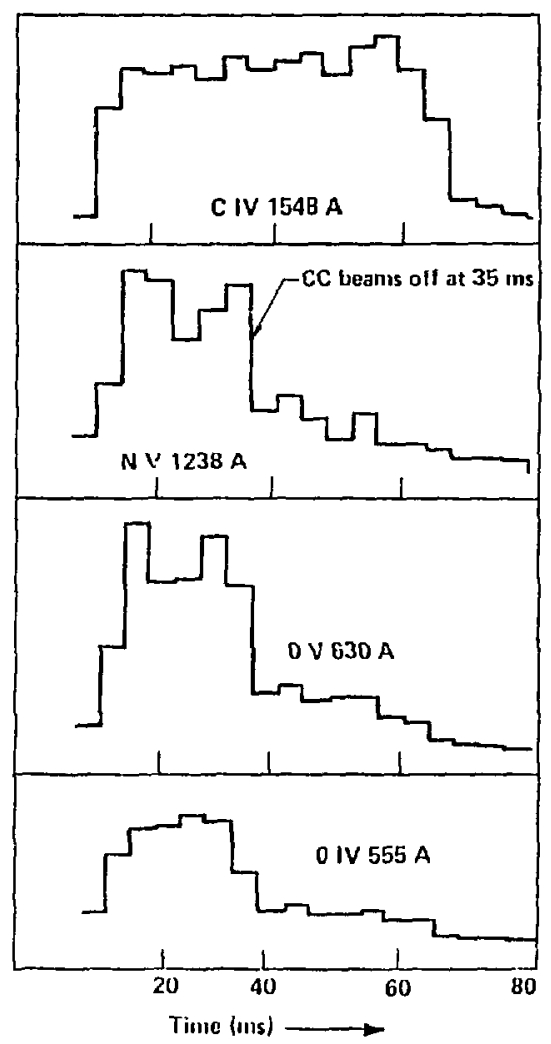

Fig. III.2 Time histories of impurity ionization states showing that oxygen and nitrogen are associated with neutral beams whereas carbon is not. 


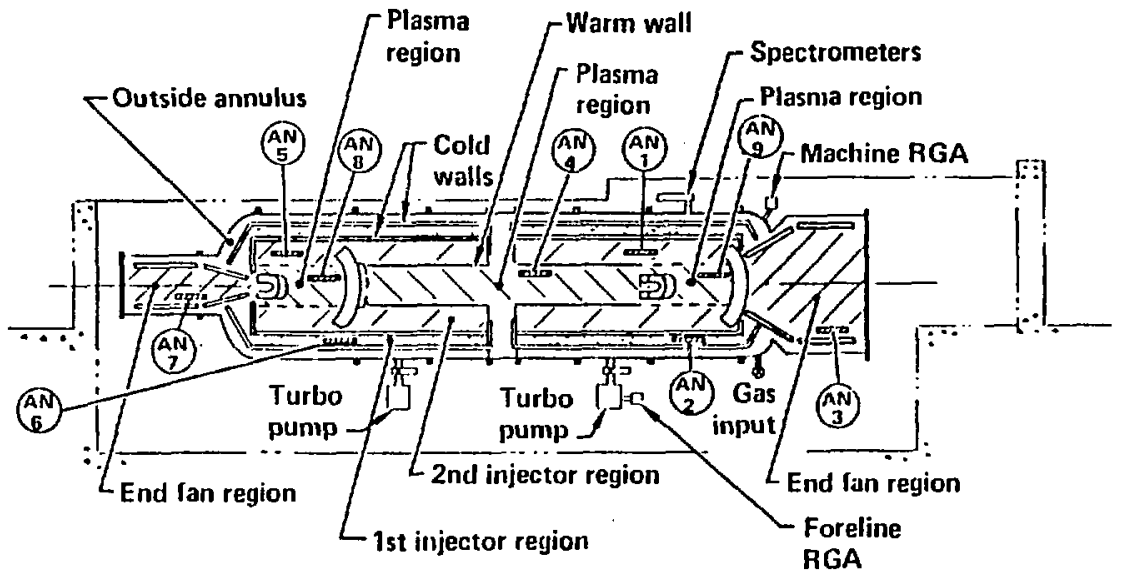

Fig : 1.3 The TMX-U vacuum vessel and glow-discharge cleaning system. The cold walls are liquid-nitrogen-cooled. The glow discharge cleaning anrode locations are labeled AN 1 to AN 9. Thanium getters are placed in between each set of walls. 


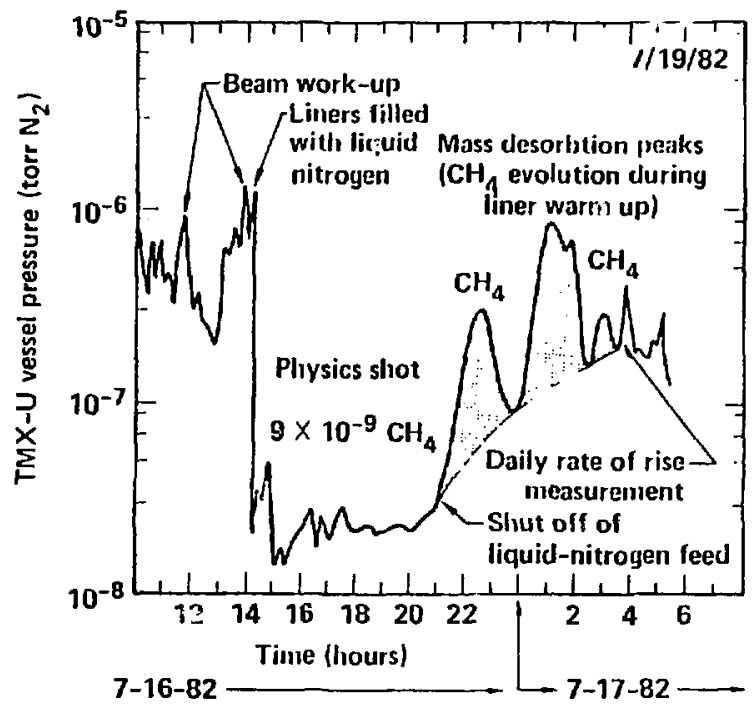

Fig. Ill.4 Pressure-time profile during a TMX-U run. The pressure increases when the liquid nitrogen that is used to fill the the liners is turned off. At this time, methane that is loosely bound to the cold walls is liberated. 
impurities in the plasma. Figure III.5 shows a plot of the carbon concentration (obtained from the EUV spectrograph) in the plasma vs. shot number for a day's run. The plasma walls were glow-discharge cleaned but not gettered. We can see from this figure that the carbon concentration decreases with shot number at the beginning of the run, showing that the plasma wall is cleaning up during initial operation. After several shots, the carbon concentration remains constant under similar running conditions.

We can control the source of the hydrocarbons in the machine by glow discharge cleaning(GDC) and titanium gettering the plasma wall. Without glow-discharge cleaning and titanium gettering before a plasma shot, the carbon impurities in the plasma are so abundant that plasma startup is difficult 68 . We observed that before GDC, the neutral beams could not sustain the plasma. This can be explained bv the observed carbon emissions; we found that the total radiated power ustimated from the measured carbon brightnesses in the central cell was a significant fraction of the estimated total input power to the plasma from the neutral beams. After GDC, the impurity radiation decreased and the electron temperature increased with ECRH-generated plasmas and the plasma would be sustained by neutral beams. (Note that as $\mathrm{T}_{e}$ increases ( $>20 \mathrm{eV}$ ), the ionization states of carbon will shift from C III and C IV to the He-like CV which does not radiate at the current temperature ${ }^{69}$.) After glow discharge-cleaning of the vacuum chamber of TMX-U, the carbon emissions decreased significantly. Table III.2 and Table III.3 present the effect of titanium gettering. The input parameters in the two shots were the same except that one shot was taken before the plasma region was gettered whereas the other shot was taken after the plasma region was titanium-gettered. The observed carbon emissions decreased by about a factor of five when the gettering was turned on. Furthermore, the decrease persisted for several shots after the gettering was stopped as shown in Table III.3. We can also see from the tables that titanium gettering shifted the ionization balance to higher ionization states showing that the $\mathrm{T}_{e}$ of the plasma increased after the gettering. The glow discharge cleaning also showed this effect.

Figure Ill.6 summarizes the trend of the carbon impurities under different 


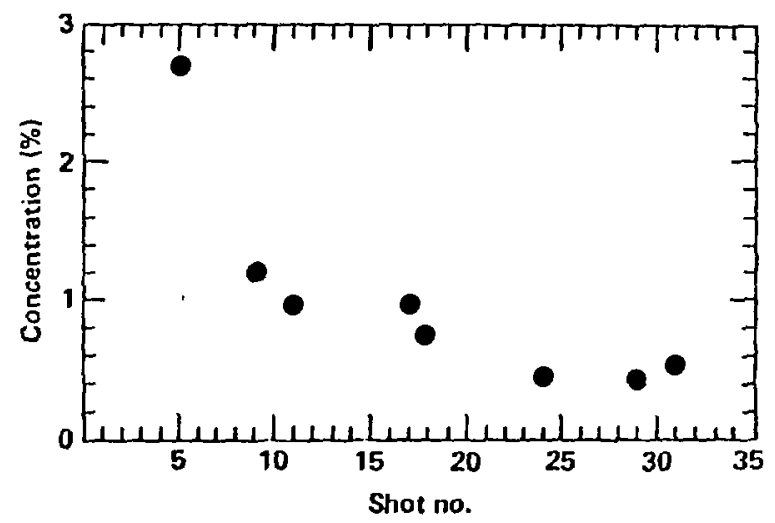

Fig. Ill.5 Carbon concentration vs. shot number for a running day. The He-like $C V$ is not induded in the catculation of total carbon. The plasma wall was glow-discharge cleaned but not gettered. 
Table III.2 Effect of gettering of plasma region on impurity concentrations (date 1/20/83, 31-35 ms, central cell).

\begin{tabular}{|c|c|c|c|c|c|}
\hline \multirow{2}{*}{ Shot no. } & \multicolumn{3}{|c|}{ Densities $\left(\times 10^{9} \mathrm{~cm}^{-3}\right)$} & $n_{O} V_{1}$ & \multirow{2}{*}{ Plasma region } \\
\hline & Carbon & Nitrogen & Oxygen & nolv & \\
\hline 7 & 25 & 5 & 37 & 1.9 & not gettered \\
\hline 8 & 5 & 8 & 26 & 3.0 & gettered \\
\hline
\end{tabular}


Table III.3 Persistence of decreased impurity densities after termination of gettering (same condition as in Table III.2).

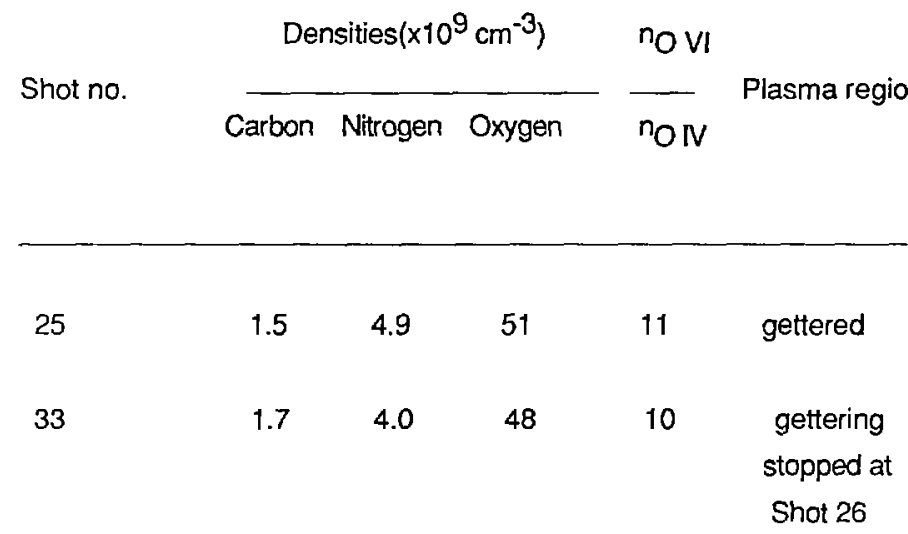


machine conditions in the wall-conditioning-study experiment. In the figure, Sequences $A$ and $A^{\prime}$ were the first stages of the experiment which were carried out immediately after the vacuum vessel was pumped out and the machine had not been exposed to GDC; gettering was used only for the two liquid-nitrogen-cooled liners. In Sequence A, ECRH-generated plasma targets were used while in Sequence $A^{\prime}$, stream-gun plasma targets were employed. in this stage, the neutral beams would not sustain the plasma. In Sequence $A$, the electron temperature was very low; resulting in very low carbon emissions; note that very little $C \mathrm{~N}$ emissions were observed. In contrast, during the plasma stream gun operation Sequence $A^{\prime}$, the carbon emissions were substantially higher and the carbon line ratios indicated that the electron temperature was considerably higer than that in Sequence $A$. The numbers in Figure III. 6 next to each data point are the brightness ratio of C IV to CIII; they are very crude indicators of the electron temperature. In contrast to the ECRH-generated plasmas in Sequence A, previous EUV measurements have shown ${ }^{52}$ that the initial electron temperature is higher during plasma-gun operation. The magnitude of the observed carbon emissions may explain why the neutral beams could not sustain the plasma during Sequence $A^{\prime}$; the total radiated power estimated from the measured carbon brightnesses in the central cell is an appreciable fraction of the estimated total input power to the electrons from the neutral beams. After sequence $A^{\prime}$, the machine walls were glow-discharge cleaned for about thirty two hours followed by Sequence $B$ with ECRH target plasmas. During this Sequence, at the beginning, the carbon brightness was comparable to that during the plasma gun shots before GDC in Sequence A'. After only a few shots, the carbon level decreased while the ratio of C IV to C III increased, indicating an increase in the electron temperature. During this period, the neutral beams began to sustain the plasma, presumably due to the decrease in the power radiated by the carbon impurities in the central cell. Sequence $\mathrm{C}$ demonstrated the effectiveness of mutiple plasma shots on impurities; the observed carbon emissions decreased by a factor of 3 to 6 during the course of 30 plasma shots in this sequence. This is followed by Sequence $\mathrm{D}$ during which the plasma walls were titanium gettered before 
Brightness

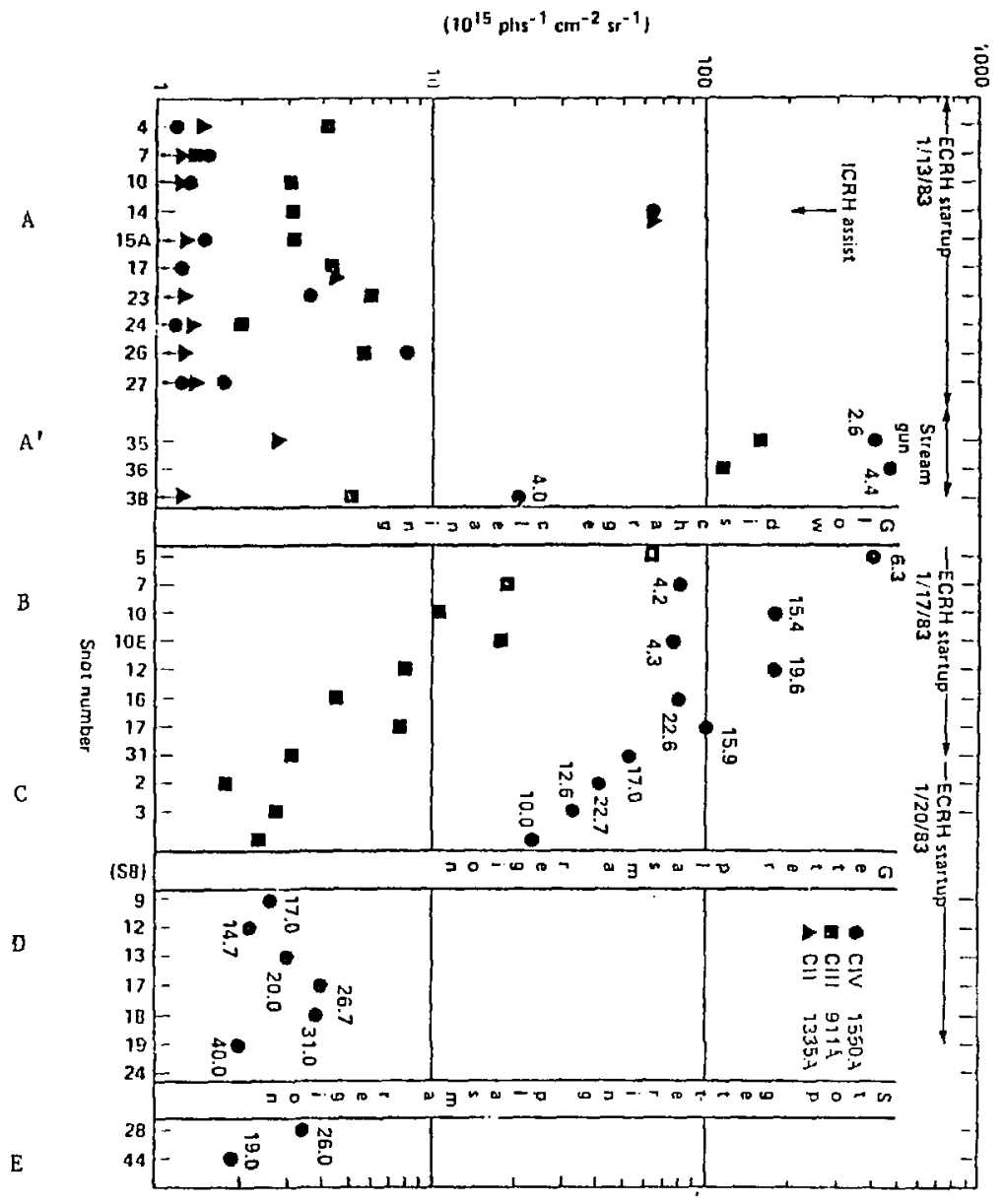

Fig. Ill.6 The brightness of carbon emissions as function of shot number for the various experimental sequences --

Sequence A-A' : before GDC;

$B-C$ : after GDC for cleanup;

$D$ : after gettering;

E : stop gettering. 
every plasma shot. The $C$ IV brightness decreases by an order of magnitude, and the CIV to C III line ratio increases somewhat. Finally, this is followed by Sequence $E$ during which a series of plasma shots without gettering was carried out and as shown in the figure, the improved conditions in Sequence $D$ persisted for several shots after the gettering was eased.

Silicon emissions were also identified during some experimental runs. In a few shots, we observed a large amount of silicon when a quartz window of the gyrotron heating system was damaged. In this way the silicon emissions act as monitors of the condition of the ECRH windows. Because the silicon emissions appear only occasionally, silicon impurities do not cause serious problems in TMX-U.

We also observed that the emission-line brightnesses of several ionization states of carbon increased with increasing ICRH power in TMX-U. (The ICRH antenna was a double-loop antenna, which was titanium gettered ${ }^{70}$.) Similar behavior of titanium emissions has also been observed by the grazing incident spectrometer mounting on the central cell of $T M X-U^{71}$. The carbon emissions are relatively insensitive to electron temperature in our experimental range (the excitation rates of C III and C IV change less than $20 \%$ over this range). Therefore, we calculated the the carbon concentrations for these shots with a constant electron temperature of $50 \mathrm{eV}$. Figure III.7 shows the carbon concentrations thus obtained vs. the corresponding ICRH power. We see that the carbon concentrations were very low in these shots and showed a general increase with the ICRH power. Due to the uncertainties in the calculations, the exact relationship between the two is not known. At present, the radiated power due to impurities for this operation does not play a role in the overall power balance.

Finally, we would like to point out that the presence of a hydrogen "impurity" during deuterium plasma operation can also have significant influence on the behavior of the plasma. For example, the transport of hydrogen ions is different from that of deuterium ions as the atomic weights differ by a factor of two. This can affect our conclusion of the behavior of the plasma ions. Previous measurements from RGA 72 had shown that after GDC and 


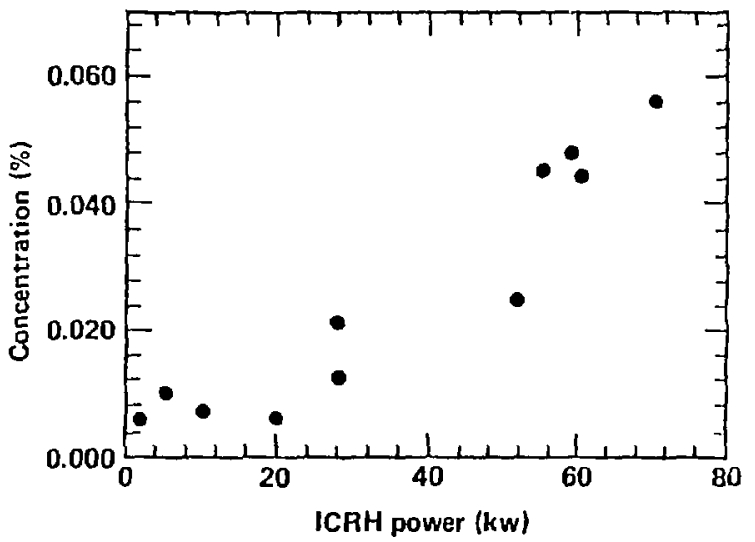

Fig. III.7 Carbon concentration vs. ICRF power showing that carbon concentration increases with the ICRF heating power. 
titanium-gettering of the walls, there were still an appreciable amount of hydrogen gas in the machine even though the plasma was directly fueled with deuterium; the abundance of hydrogen gas decreased with each day of plasma operation. Recent results from end loss ion spectrometer ${ }^{73}$ showed that during the first few titanium-gettered shots, the fraction of hydrogen plasma ions may be as high as $30 \%$. This fraction decreased to an unmeasureable level after several plasma shots with gettering which might have removed water in the unbaked TMX-U vacuum vessel. This is consistent with the measurements of titanium film of the TMX-U wall ${ }^{74}$ in which the main consistuent was oxygen.

\section{III.4 Accumulation of Impurity lons in Thermal Barrier Region}

As discussed before, a thermal barrier is a region of negative potential between the central cell and the end-plug positive potential (Fig. I.1); it is formed by mirror-trapping a population of energetic electrons. The barrier can isolate the thermal plug electrons from the central cell so that the plugging potential can be raised substantially by heating the plug electrons locally.

Unless the thermal barrier is infinitely "deep", there are always some electrons passing from the central cell to the thermal barrier region. The relation between the densities of these electrons at the barrier region and the central cell is governed by the Boltzmann relation

$$
e \phi_{b}=T_{e c} \ln \left(n_{c} / n_{b}{ }^{*}\right)
$$

where $T_{e c}$ is the central cell electron temperature, $n_{c}$ is the central cell 
electron density, and $n_{b}{ }^{*}$ is the passing electron density at the barrier point (the total electron density at this point is equal to the sum of the passing electron density and that of the hot-electron density ). However, the plug electrons are not in local thermodynamic equilibrium; the Boltzmann relation does not apply to potential variations and densities in the region between the barrier and the end-plug potential.

The ions can fill in the thermal barrier (subsequently destroying it) by collisional-scattering into the "trapped" regime. The trapped ion density must be maintained at a low level by pumping the ions out of the barrier through charge exchange. The ion trapping rate expression in a barrier has been obtained by fitting the plasma parameters over a variety of square-well Fokker-Planck computations 75 . Specifically, models of impurity accumulation in a tandem mirror has also been developed 66 . Besides collisional-scatter into the "trapped domain", impurities can also be trapped if they are ionized to higher charge state at the barrier region. (Radial transport could reduce significantly the impurity accumulation in the barrier ${ }^{66}$ ). Because impurity ions are multiply charged, they are very effective in filling up the barrier. This increases the required pumping beam current, the loss rate of the sloshing ions, and the energy loss through impurity radiations. Therefore, the impurity concentration must be maintained at a low level to avoid the filling up of the barrier.

However, the model 66 predicts that under the current conditions with a barrier depth $500 \mathrm{eV}$ and average charge state equal to 4, the impurities in TMX-U would not fill in the thermal barrier with a significant rate. We have conducted experiments to test this fact. Neon gas was puffed into the east end plug through a fast valve; the 22-channel monochromator was looking at the barrier region at the east end plug and monitored the variation of the neon ion signals during the formation of the thermal barrier. We found that there was no significant change in the neon signal. Also, under current operating conditions, intrinsic impurities do not accumulate in the thermal barrier. Fig. Ill. 8 shows the time histories of carbon and nitrogen emissions obtained from the spectrograph when it viewed at the thermal barrier region of the west end plug. 

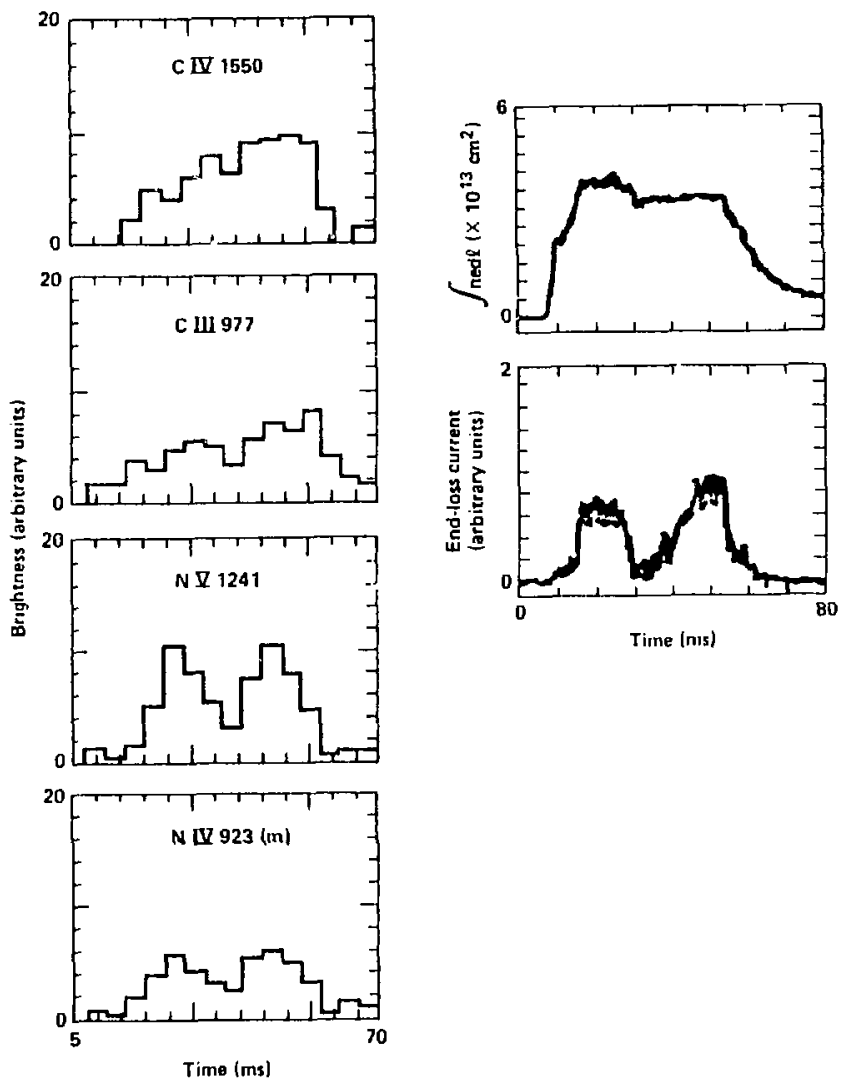

Fig. III.8 Nitrogen impurity ions at the thermal barrier region did not increase during the formation of the barrier( $30 \mathrm{~ms}$ )(Shot 4 of 12/1283). C N , C III, N V and N IV emissions measured in the west end plug are shown vs. time. The end plug line density and ion end lo:ses are also shown. Note the impurity emissions do not increase substantially during the time of enhanced axial confinement. 
The time histories of the corresponding line density $\int n_{e} \mathrm{dl}$ and end loss current at the west end plug are also shown in the figure. The decrease of the end loss current to zero around $30 \mathrm{~ms}$ showed that axial confinement increased. As shown ir. the figure, the line density remained unchanged during this time interval. A change in the impurity signal (on the left side of the figure) which is proportional to the product of the line density and the impurity density therefore reflects the change in the impurity abundance. There was no significant increase in the impurity signal during this time interval; this implies that no relevant accumulation of impurities had occured. In future mirror experiments such as MFTF-B, the electron temperature will be much higher and the barrier depth will be larger; the trapping rate of impurities is expected to be much more pronounced. 


\section{III.5 Measurement of the Kinetic Energy of Impurity lons by the Doppler Broadening}

We can use the high-resolution ( 0.7 A) gratings of the normal incidence spectrograph to estimate the kinetic energy of the impurity ions from the Doppler brcadening of appropriate emission lines. Shown in Fig. III.9 are two spectra of two different shots taken with grating $F(1100-1400 \AA)$. The doublets NV 1239-1243 $\AA$ are well resolved in Figure III.9a (Shot 1), while they are blended with each other in Figure III.9b (Shot 2). This is because unlike Shot 1, the $N V$ ions in Shot 2 are energetic and their emissions are Doppler-broadened. Shot 2 was operated under normal conditions with central ceil neutral beams turned on . During the operation of Shot 1 , a large gas leak occured and the nitrogen came from the room-temperature air and thus was very "cold". Therefore, the emissions from the nitrogen ions in Shot 1 did not show any Doppler broadening and the $N V$ doublets were wall resolved.

We can estimate the kinetic energy of the N V ions from the Doppler broadening of these doublets. If there were no line broadening, the doublets should be separated by about $4 \AA$ as in Shot 1 . In Shot 2 , the doublets overlapped each other, we conclude that the broadening of them was about 1 to $2 \AA$. A more precise method to obtain the Doppler width is discussed as follow.

In general, the line shape arising from Doppler broadening and instrumental effects can be represented by a Voigt profile (the convolution of a Gaussian and Lorentzian shape) ${ }^{76-78}$,

$$
I_{v}(\lambda, \alpha, \beta) \approx \int_{-\infty}^{\infty} \frac{e^{-(\lambda-\lambda)^{2} / \alpha^{2}}}{\lambda^{2}+\beta^{2}} d \lambda^{\prime}
$$

because to a good approximation, the instrumental line shape is Lorentzian. 

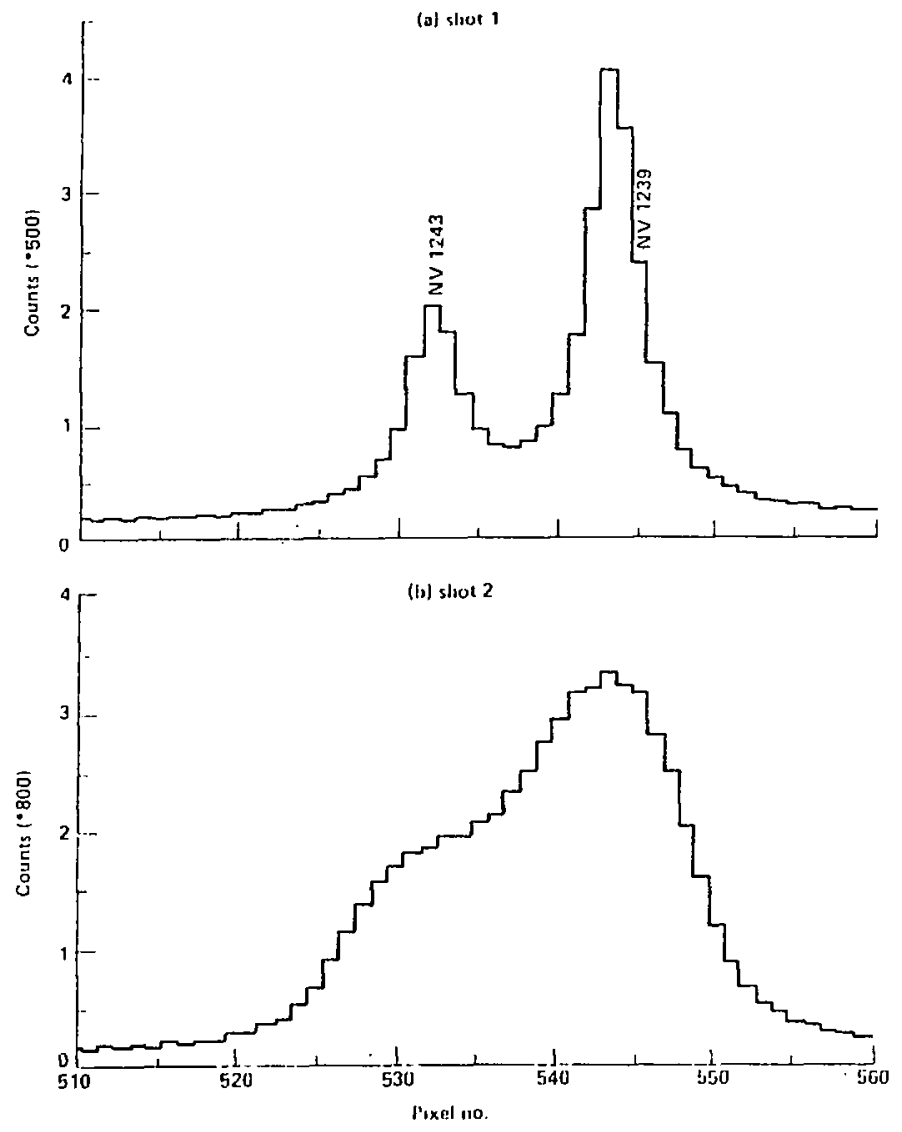

Fig. III.9 Spectra showing the effect of Doppler broadening of NV 12391243 A. (a) This spectrum which does not have Doppler broadening was taken when there was a large gas leak during the shot thus introducing cold nitrogen. (b)This spectrum which shows the Doppler broadening effect was taken when the CC neutral beams were on. 
In expression (III.2), $\alpha$ is the corresponding Doppler width, and $\beta$ is the corresponding Lorentzian width. The profile of the $\mathrm{N} V$ doublets can be expressed as

$$
I_{D}(X, \alpha, \beta)=I_{V}(X-X 1239, \alpha, \beta)+0.5 I_{V}(X-X 1243, \alpha, \beta)
$$

where $X 1239$ and $X 1243$ are the predetermined positions (pixel number) of the lines $\lambda 1239$ and $\lambda 1243$ respectively. Our goal is to determine the value of $\alpha$ from which we could deduce the ion kinetic energies. If $\beta$ is known, we can perform a least-squares-fit to the data points of the doublet profiles using equation (III.3) with $\alpha$ as the only fitting variables. In the fitting process, the $I_{v}(X, \alpha, \beta)$ values can be obtained by performing numerical integrations of expression (III.2). Suppose $\alpha_{0}$ is the value that gives the best fit to the data points, then we can determine the average kinetic energy $E_{i}$ of the $N V$ ions by

$$
E_{i}=\left(M c^{2}\right)\left(\alpha_{o} / \lambda_{0}\right)^{2} / 2
$$

where $M$ is the atomic mass of the ion; $c$ is the velocity of light; and $\lambda_{0}$ is the wavelength of the line considered. More precisely, an adjustment factor of the order of 1 should be multiplied to the right side of (III.4); the exact value of which depends on the actual distribution of the emitting ions.

In this method, the Lorentzian width \& in (III.2) has to be known a priori. It can be determined by making use of a special case in which.the Doppler broadening is negligible like the case of figure III.9a discussed above. Under such a condition, we could let $\alpha \rightarrow 0$, and again perform a similar least square 
fit to the doublets shown in figure III.9a but now, 8 is the fitting variable irstead of $\alpha$. The required $\beta$ is the one which best fits the data points of the $N \vee$ doublets shown in figure Ill. 9 a.

Using this method, in many cases, we obtained Doppier width values about $1.5 \AA$ which are consistent with the rough estimation discussed before. This value of broadening would correspond to a kinetic energy of the order of $10 \mathrm{keV}$. This is additional evidence that the nitrogen ions were associated with the neutral beams.

Figure III.10 shows the time history of the N V ion energy obtained from the Doppler broadening. The energy decreased significantly after the neutral beams were turned off. The uncertainty of each of the $E_{j}$ values of the data points in the figure is about $50 \%$. However, the uncertainty of the relative energy between two data points should be less than $30 \%$. 

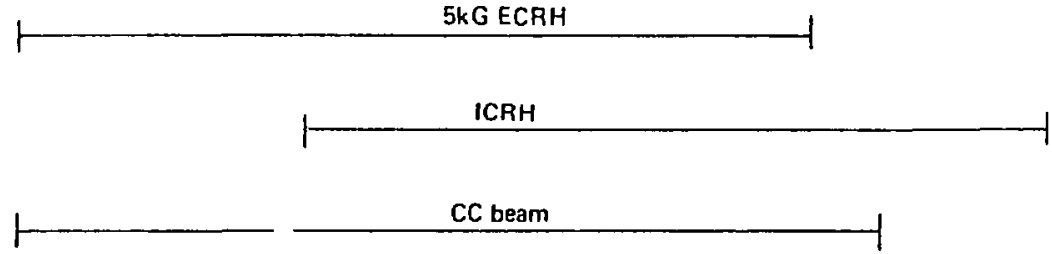

$\mathrm{CC}$ beam
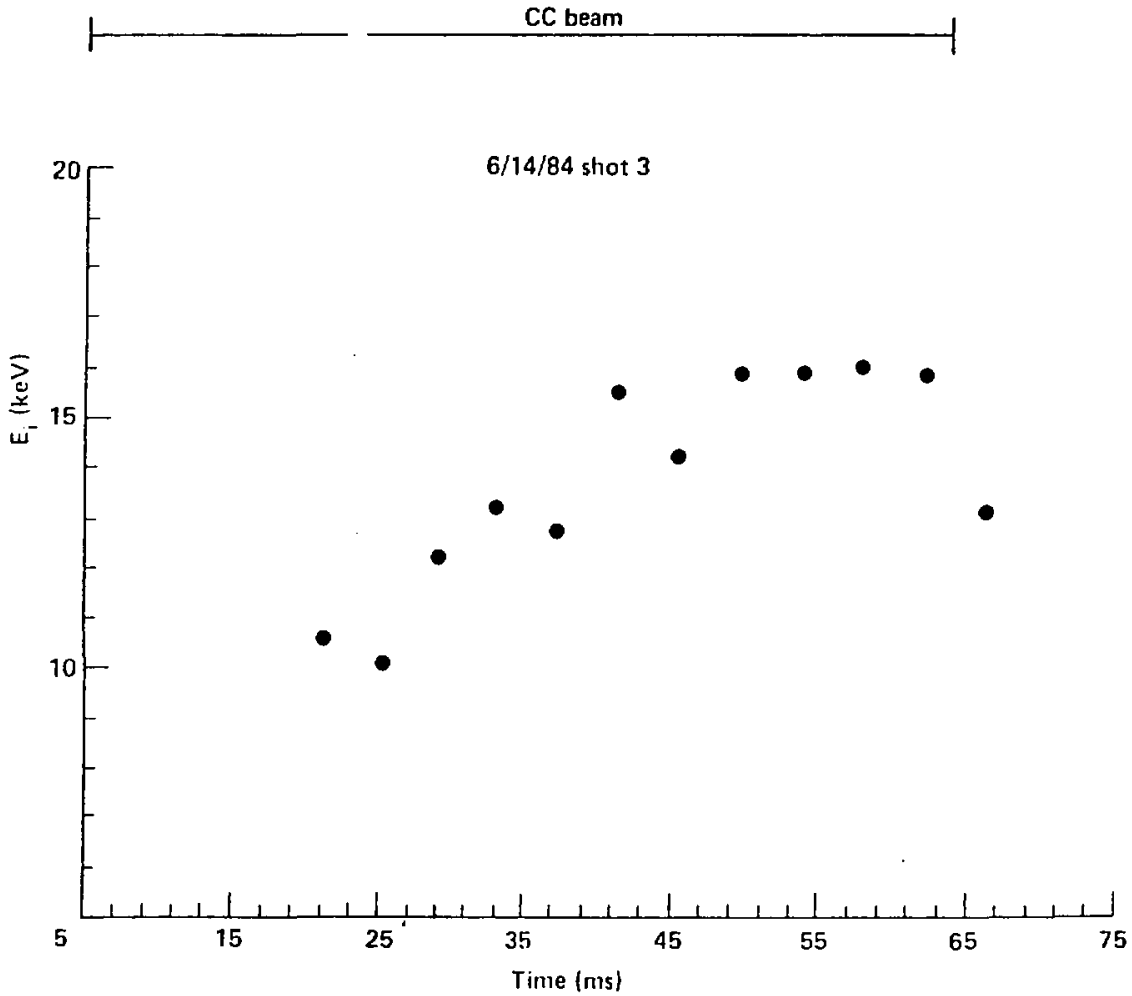

Fig. Ill.10 Kinetic energies of N V ions obtained from Doppler widths vs. time. The ions energies decreased when the $\mathrm{CC}$ beams were turned off. 


\section{III.6 Estimation of the Fueling Rate from Ly $\propto$}

Ly $\alpha$ is a bright emission line at $\lambda 1216$ from neutral hydrogen or deuterium. The excitation coefficient 52 of Ly $\alpha$ is very similar to the ionization coefficient ${ }^{79}$ of deuterium (i.e. $\langle\sigma v\rangle_{e x} \simeq\langle\sigma v\rangle_{\text {ion }}$ ). Utilizing this fact, we can estimate the fueling rate of the plasma from the measured brightness of $L y \propto$ which can be expressed as

$$
B=(1 / 4 \pi) \int<\sigma v>e^{n} e^{n_{D}} d
$$

In (III.5), the integration is performed along a line of sight of the instrument; $\mathrm{n}_{\mathrm{e}}$ and $\mathrm{n}_{\mathrm{D}}$ are the densities of electrons and deuterium respectively. On the other hand, the current 1 corresponding to the rate of ionizing the neutral deuterium is giver, by

$$
\begin{aligned}
I & =e \int<\sigma v>_{i o n} n_{e} n_{D} d V \\
& =e \int<\sigma v>_{i o n} n_{e} n_{D}(2 \pi r) d r d z
\end{aligned}
$$

By assuming that $\langle\sigma v\rangle_{\text {ion }}=\langle\sigma v\rangle_{\text {ex }}$ and that the neutral deuterium is located in a shell of width $\delta r$ and offset $r_{0}$ (see Chapter IV), we can reduce (III.6) to

$$
1 \simeq 4 \pi^{2} e r L_{C} r_{o} B
$$

where $L_{c}$ is the length of the central cell plasma and $r$ is a factor arising from the integration along $z$ in case the distribution of the neutrals is not uniform in the $z$-direction. The value of $\gamma$ can be evaluated by assuming a reasonable 
distribution of $\mathrm{n}_{\mathrm{D}}$ in $\mathrm{z}$.

For example, in many cases B was measured to be $4 \times 10^{14} \mathrm{ph} / \mathrm{cm}^{2}-\mathrm{sr}-\mathrm{s}$. If we assume $\gamma=0.3, L_{c} \simeq 500 \mathrm{~cm}$, and $r_{0} \simeq 15 \mathrm{~cm}$, then we obtain a fueling rate of $\_$ 6 amperes. Two video cameras 80,81 have been mounted on the west end plug and the central cell(near the gas box) of TMX-U to measure the brightness of the visible $\mathrm{H}_{\propto}$ from the deuterium neutrals; the fueling rate is estimated from the $\mathrm{H} \propto$ brightness. The video cameras were located near the gas box and thus could obtain estimates of the fueling rates. However, the 1024 channel spectrograph can check for the axial profile of the fueling rate diagnostic; it also provides information about the neutral distribution along the axis of the machine.

In this chapter, we have discussed the general aspects of impurities in $T M X-U$. The spectroscopic diagnostic is a very powerful method in studying impurity properties in fusion machines and the understanding of the impurity behaviors can in turn shed light on the study of the plasma properties in the pursuit of making magnetic fusion feasible. In closing, we would like to mention that in some of the plasma shots, the ratios of $\left(2 s{ }^{2} S_{1 / 2}-2 p{ }^{2} P_{3 / 2}\right)$ / (2s ${ }^{2} \mathrm{~S}_{1 / 2}-2 \mathrm{p}{ }^{2} \mathrm{P}_{1 / 2}$ ) of $\mathrm{N} V$ were observed to be anomalous. Under normal conditions, this ratio is governed by the branching ratio of the two lines and is simply equal to 2. However, we have observed values as low as 0.9 (the uncertainty involved in measuring this line ratio is only about $10 \%$ ). The anomalous line ratio has been obseved under normal operations; the effect is more pronounced when nitrogen neutral bearns (instead of the deuterium neutral beams) were used. Neither the radiation absorption process nor the charge exchange effects could explain this anomalous ratio. We wish future work would clarify this discrepancy. 


\section{Chapter IV Impurity Transport in TMX-U}

\section{IV.1 Introduction}

Impurity transport in tokamaks has been studied at several different laboratories $82-88$. In many cases, laser blow-off has been used to introduce a trace amount of impurities into high density tokamaks. In some cases it was found that the transport phenomenon in tokamaks was different than theoretical predictions and the confinement times of the impurities in tokamaks were much shorter than those predicted from theory $83-84$.

The particle transport in mirror machines involves two channels of transport -- radial and axial. In early simple mirror machines 1,89 , the axial transport was larger than the radial transport. However, in recent tandem mirrors, the axial confinement has been improved and the radial transport begins to play a more important role in the particle confinement. The positive potential which exists at the two ends of a tandem mirror is particularly good at confining multiply-charged impurity ions. The parallel (axial) confinement time $\tau_{/ /}$of an impurity ion in a hydrogen plasma confined by a tandem mirror is given by 90

$$
\tau_{/ /}=\tau_{i i} z_{i} e \phi g(R) \cdot \operatorname{EXP}\left(z_{i} e \phi / T_{i}\right)
$$

$$
\text { where } \begin{aligned}
\tau_{i i} & =m_{p}{ }^{1 / 2} T_{i}^{3 / 2} / \sqrt{ } 2 \pi e^{4} z_{i}^{2} z_{e f f}{ }^{2} A_{i}^{1 / 2} n_{p} \ln \wedge \\
g(R) & =\sqrt{ } \pi(1+1 / R) \ln (2 R+2) / 2 \\
R & =\text { mirror ratio } \\
\phi & =\text { the axial ambipolar potential } \\
A_{i} & =\text { atomic mass of ions } \\
z_{i} & =\text { charge carried by the impurity ion }
\end{aligned}
$$




$$
\begin{aligned}
& T_{i}=\text { impurity ion temperature } \\
& n_{p}=\text { density of plasma } \\
& m_{p}=\text { mass of proton } \\
& z_{\text {eff }}{ }^{2}=1+\alpha_{1} z_{1}{ }^{2}+\alpha_{2} z_{2}{ }^{2}+\ldots . \\
& \alpha_{j}=\text { concentration of } j \text {-th species in the plasma }
\end{aligned}
$$

We see that $\tau_{i j} \propto z_{i} \operatorname{EXP}\left(z_{i} \Theta \phi / T_{j}\right)$ implying that $\tau_{i j}$ increases very rapidly with $z_{j}$. In these cases, we might expect that radial loss becomes the dominant impurity loss mechanism. Our experiments were motivated by the fact that the mechanisms involved in the radial impurity transport in a tandem mirror machine are not well understood.

Recent theories have shown that the presence of non-axisymmetric tandem mirrors can enhance central cell transverse losses $91-94$. This is because when a particle is reflected from a quadrupole field, the center of the Larmor circle of the particle will be displaced by an amount $\delta r$ (positive or negative), the magnitude of which depends on the energy, magnetic moment, and position of the particle. After the particle has been reflected, it approaches the other mirror on the other end of the machine. In an axial transit of the solenoid, the particle will experience an azimuthal drift caused by the radial gradient in the central cell magnetic field (i.e. $\nabla \mathrm{B}$ drift), and by electric fields due to a nonuniform radial ambipolar potential (i.e. EXB drift). Since the quadrupole field of the two mirrors is rotated by $90^{\circ}$, if the azimuthal drift is an odd multiple of $\pi / 2$, the radial displacements are in phase and superimposed constructively. This leads to resonant diffusion.

The TMX-U central cell plasma parameters are in the resonant-diffusion regime 95,96 . This kind of diffusion is nonambipolar (i.e. ions and electrons are lost at different rates ${ }^{97}$ ) which would have significant impact on the particle balance $99-100$. It has been shown that the plasma transport in both $T M X^{101-102}$ and TMX-U 103-105 has nonambipolar characteristics although 
the resonance has not been unambiguously demonstrated to be the cause.

Nonambipolar impurity Iransport has implications on the total particle balance. For example, consider a plasma which consists of $2 \%$ of five times ionized ions which are lost nonambipolarly in the radial direction. To maintain the quasineutrality of the plasma, abou: $10 \%$ of the excess electrons have to be lost axially at the same rate as the impurity ions. This could give rise to a relatively large net negative end loss current if the confinement of the impurity ions is worse than that of the plasma ions as in the case of TMX 59 .

Results from impurtiy transport studies may shed light on the bulk plasma behavior and test general theories of particle transport and diffusion ii a magnetized plasma. In order to study the impurity transport in TMX-U, we employed two neutral beams to inject energetic neutral nitrogen ( $>10 \mathrm{keV}$ ) into the central cell at a plasma density of $4 \times 10^{12} \mathrm{~cm}^{-3} 106-107$. (We fueled the gas chamber of each of the neutral beam injectors with nitrogen gas instead of the usual deuterium gas. A detailed description of the neutral beam injection system can be found in refert. jes 108 and 109.) This experiment was first performed in Febuary 1984 and was repeated in June 1985. This will be discussed in section IV.2. We also investigated the transport of intrinsic impurities in section IV.3.

\section{IV.2 Global Confinement Time of Beam-injected lons in TMX-U}

In the nitrogen beari injection experiment, different ionization states of nitrogen were observed by the time-resolving 1024-channel spectrograph at the central cell. In some shots of the experiment, pixel selection was used to read out subsets of the the 1024 channels to focus on nitrogen ion emissions. This enabled us to increase the time resolution of the spectra to about $1.2-2 \mathrm{~ms}$; the exact value depended on the number of lines selected. Radial scans were obtained on a shot to shot basis. In the east end plug, emissions from NIV and NV ions were observed with the 22-channel monochromator. 
In Febuary, 1984, during the injection experiment, the electron densitiy profiles were measured by the Beam Attenuation Detector $(B A D)^{110}$. The electron density profile was consistent with a Guassian with a peak density $\mathrm{n}_{\mathrm{eo}}=$ $4 \times 10^{12} \mathrm{~cm}^{-3}$ and $1 / e$ radius $r_{C}=20 \mathrm{~cm}$. The nitrogen beam current trapped in the central cell plasma was also measured by $B A D$ and was found to be $I_{N} \sim 1.1 \mathrm{~A}$. We also used the method discussed in Chapter III to estimate the total concentration of nitrogen $\mathrm{C}_{\mathrm{N}}$ from the nitrogen spectrum during the "steady state" of injection. We found that $C_{N}$ was approximately $1 \%$. From this information, we estimated the global confinement time of nitrogen $\tau_{N}$ in the CC plasma.

$$
\begin{aligned}
& \text { i.e. } \tau_{N} \sim\left(n_{e o} C_{N}\right)\left(\pi r_{C}{ }^{2} L_{C}\right) e / l_{N} \\
& \sim\left(4 \times 10^{12 *} 0.01\right)\left(\pi 20^{2 *} 500\right) 1.6 \times 10^{-19 / 1.1} \\
& \sim 4 \mathrm{~ms}
\end{aligned}
$$

In Eq. IV.2, $L_{C}=$ effective length of plasma in the central cell and $e$ is the electron charge. The $4 \mathrm{~ms}$ confinement time thus obtained is of the order of magnitude of the confiment time for the bulk plasma of TMX-U ${ }^{111}$. Similar values were also obtained for the experiments done in June, 1985. Note that the $4 \mathrm{~ms}$ given in Eq. IV.2 includes both the parallel and radial confinement time.

We also noticed that the net negative current measured toj the plasma potential control (PPC) plates had no significant increase when the $N$-beams were turned on. We might therefore conclude that there was no significant non-ambipolar transport of the impurity ions. In addition, we compared the decay characteristics of the different nitrogen ions with simple simulations to obtain an order-of-magnitude-estimate of the continement time. Fig. IV.1 presents the time histories of the emissions from different nitrogen ionization states (time resolution $\sim 2 \mathrm{~ms}$ ). Fig. IV.2 shows the time evolution of $\mathrm{N} V 1240$ line at a higher time resolution ( 1.2 ms). These curves are results of the experiments 

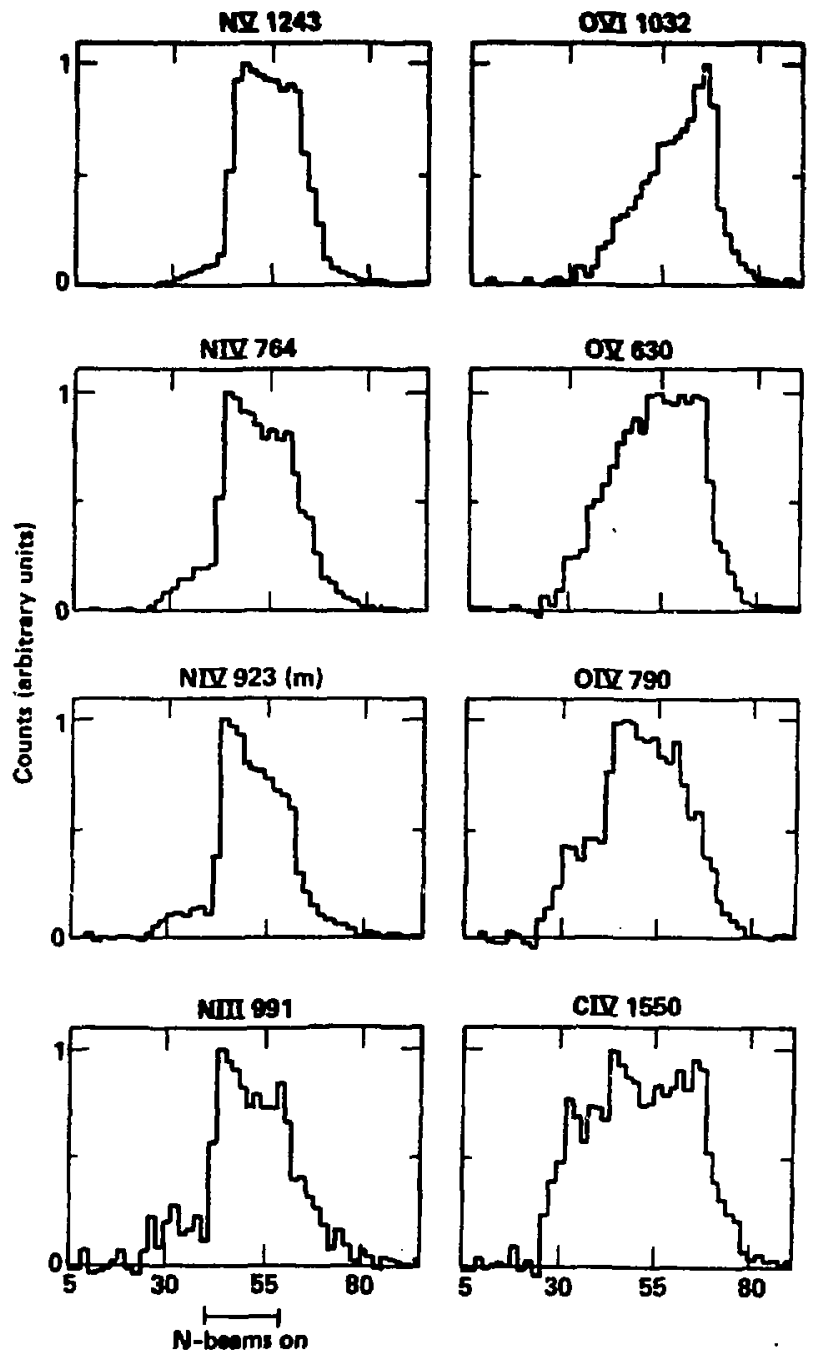

Time (ms)

Fig. IV.1 Time histories of different ion emissions (time resolution $\sim 2 \mathrm{~ms}$ ) in CC during nitrogen injection. 


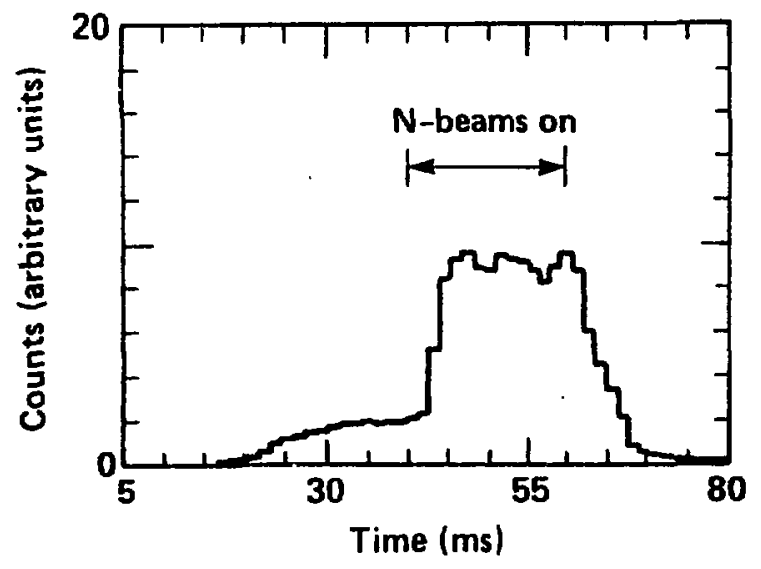

Flg. IV.2 Time histories of N V 1239 (time resolution $~ 1.2 \mathrm{~ms}$ ) in ᄃC during nitrogen injection. 
in Febuary, 1984 during which the larger preslit was used to obtain larger signal and no appropriate radial scan was obtained. Note that different ionization states have different rise and decay times. The signils of higher ionization states rose slower than than those of lower ionization states. The emissions appeared in order of increasing ionization state. (This phenomenon was also true for intrinsic impurities at the end plug. Fig. IV.3 clearly shows this effect ${ }^{62}$.) This effect can also be seen in Fig. IV.4 which presents approximate simulated time profiles of nitrogen ions under the conditions that nitrogen neutrals were injected into the plasma without disturbing the plasma (i.e. electron temperature and density wcre kept constant). We will consider the decay ch aracterisics instead of the rising characteristics because at the beginning of the injection, there might be rapid changes in the radial profiles of the nitrogen ions which leads to difficulties in interpreting the recorded physical data precisely.

By neglecting the recombination rates of various nitrogen ionization states and making certain reasonable assur, stions, we were able to obtain analytic expressions for the time histories of the ions. The anlaytic expressions and details for deriving them are presented in Appendix B. The curves shown in Fig. N.4-5 were generated using these analytic formulas. We have assumed that continement times for different ionization Siates were the same and are constant in radius. This is similar to observations on tokamaks ${ }^{83-84}$. Fig. N.4-5 show two sets of curves with two different confinement times for the nitrogen ions at a central temperature of $30 \mathrm{eV}$ which was the approximate temperature measured by Thomson scattering in Febuary, 1984. We compared the analytic curves with the actual time histories of brightnesses emitted by nitrogen ions when $\mathrm{N}$-beams were turned off. We concluded that the confinement time for the beam-injected nitrogen ions lied between 1 and $10 \mathrm{~ms}$. This is consistent with the value of $4 \mathrm{~ms}$ that we obtained earlier. 


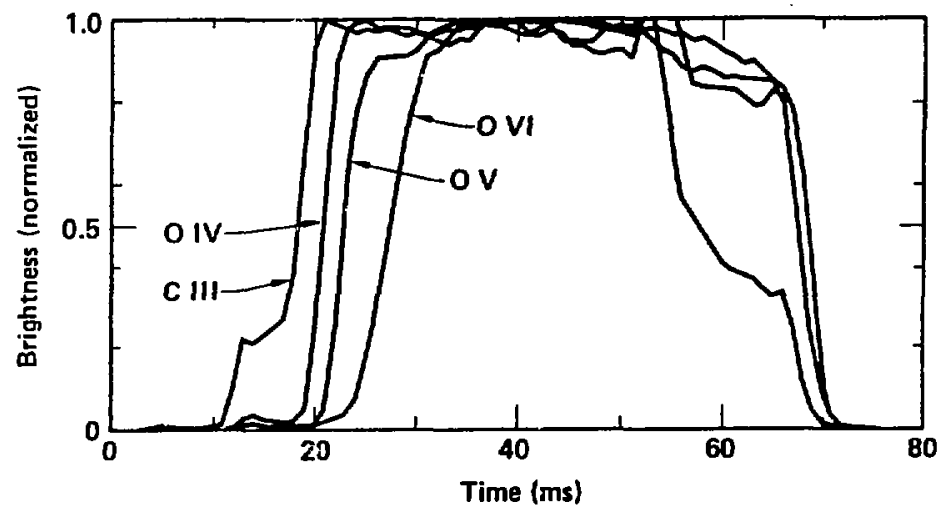

Fig. IV.3 Time histories of different ion emissions in the east end plug 62 . 

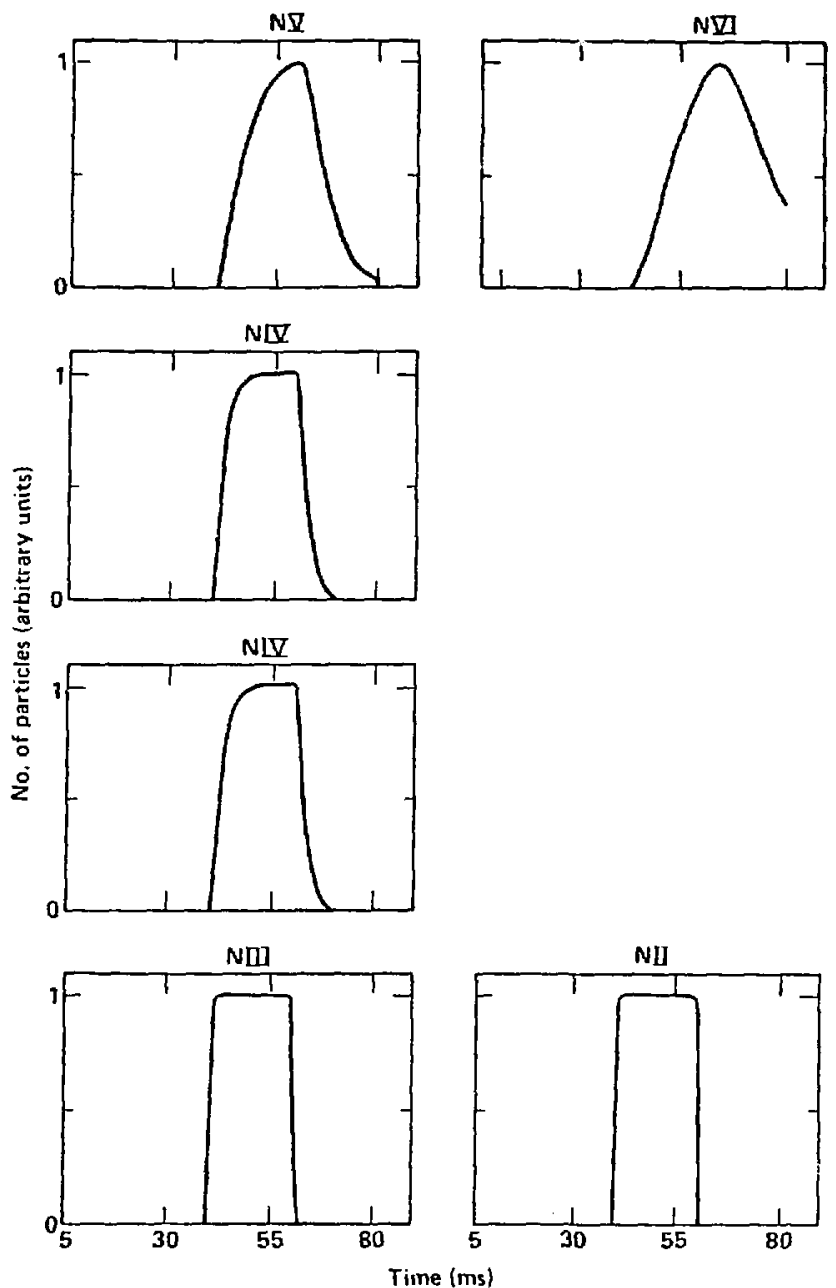

Fig. IV.4 Time profiles of nitrogen ions obtained from analytic equations described in Appendix B with electron temperature $30 \mathrm{eV}$ and confinement time $10 \mathrm{~ms}$. 
No. of particies (arbitrary units)
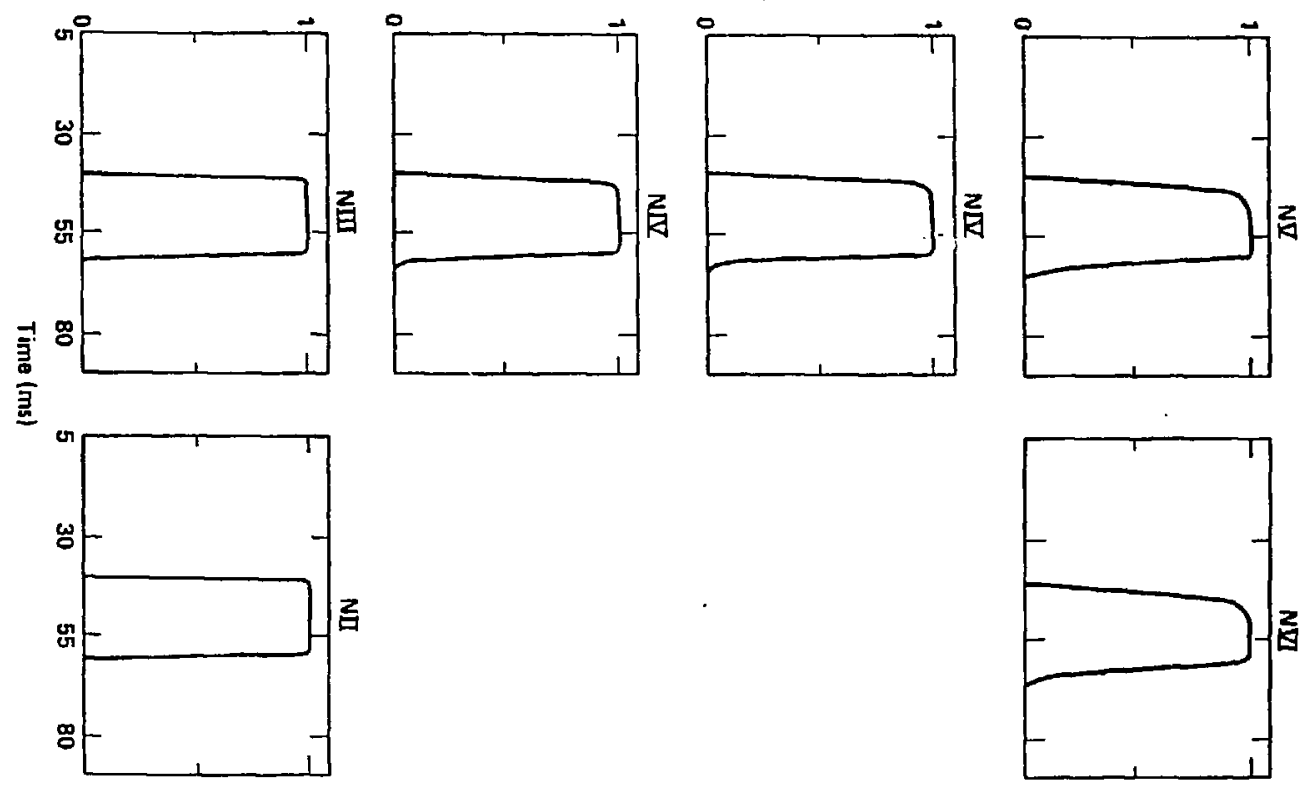


\section{IV.3 Radial Transport of Beam-injected and Wall-originated Impurities}

During the constant-condition run in Febuary, 1985, we were able to obtain radial scans of $\mathrm{C}, \mathrm{N}$ and $\mathrm{O}$ ion emissions in the central cell on a shot by shot basis. Similar scans were also obtained for nitrogen during the $\mathrm{N}$-beam-injection experiment in June, 1985. The results are shown in figures IV.6-9. In general, the brightness data can be Abel-inverted ${ }^{112}$ or geometrical-inverted ${ }^{113}$ to obtain the volume emission profiles from which we could deduce the radial profiles of the ion densities. However, when the data points are inverted, the error of the data points near the "edge" ( larger radius) "propagates" towards the center ${ }^{114-115}$. This makes the uncertainty of the inverted data near the center very large. Therefore, we have adopted a different approach here. We assumed that the $\mathrm{T}_{e}$ and $\mathrm{n}_{e}$ profiles could be represented by Gaussians.

i.e. $\quad n_{e}=n_{e o} \cdot \operatorname{EXP}\left(-r^{2} / r_{c}{ }^{2}\right)$

$$
T_{e}=T_{e o} \cdot \operatorname{EXP}\left(-r^{2} / T_{T}^{2}\right)
$$

The quantities ${ }^{c},{ }^{\prime} T, n_{e o}$, and $T_{e o}$ were considered known. For simplicity, we assumed that each of the ion density profiles, $n_{i}(r)$ could be represented by a Lorentzian with width $D_{i}$ and offset $r_{i o}$ from the axis.

$$
n_{i}=\frac{n_{i 0}}{1+\left(r-r_{i 0}\right)^{2} / D_{i}^{2}}
$$

The brightness of an emitting line from an ion species was thus given by 

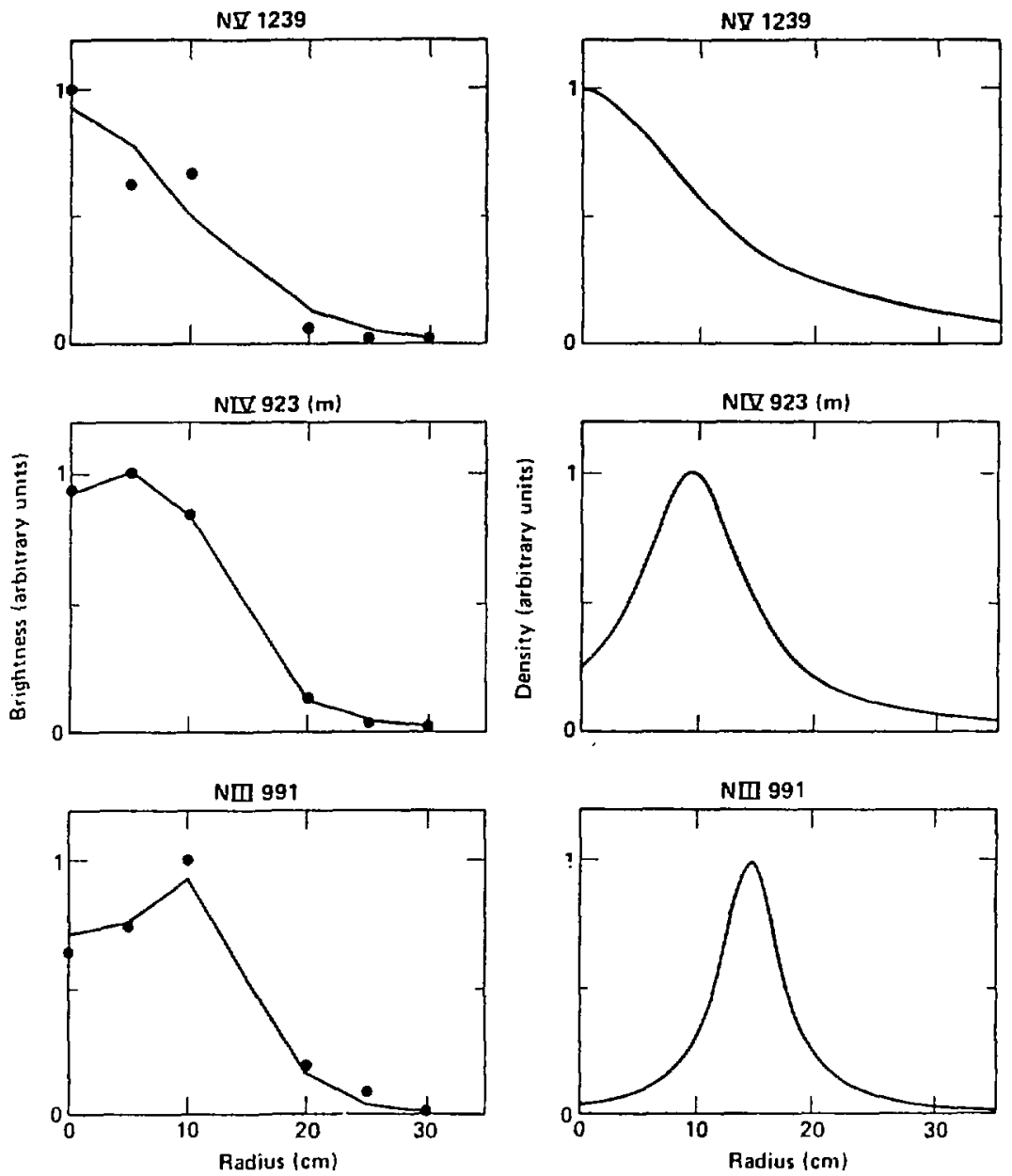

Fig. IV.6 Radial profiles of N III to N V ions. The solid dots represent the real brightness data points at different chord positions. The solid lines on the left column are the "fitted" brightness values (see explanation in text). The right column shows the density profiles which best-fit the corresponding brightness dala points shown on the left column. 

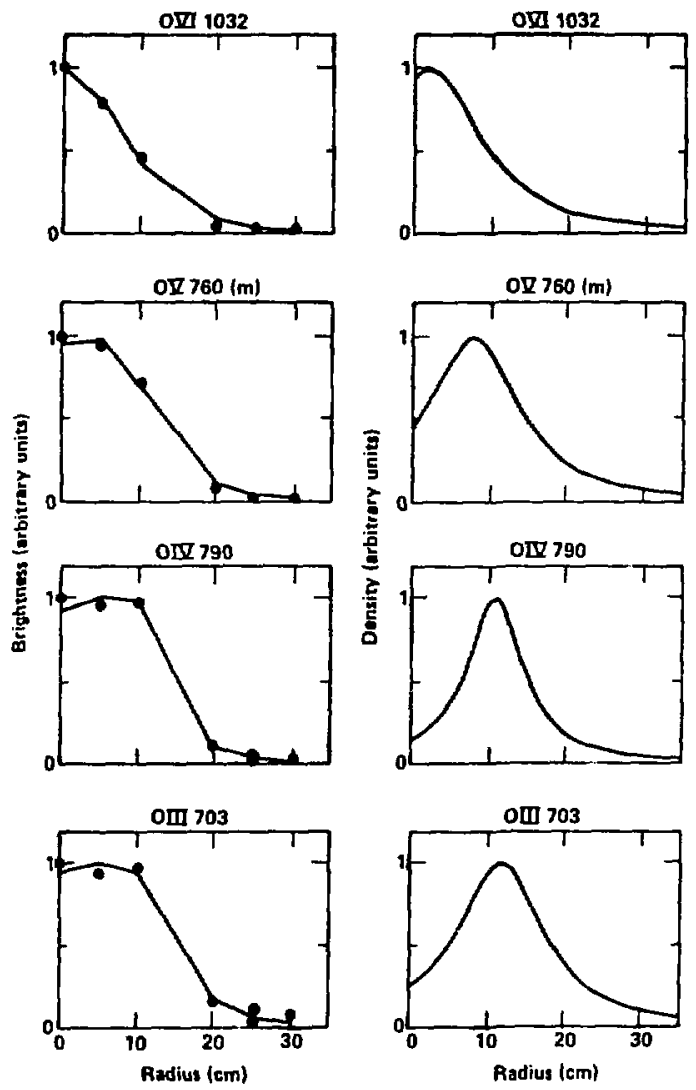

Fig. IV.7 Similar to Fig. IV.6 except that now the profiles of O III to O VI emissions are shown. 

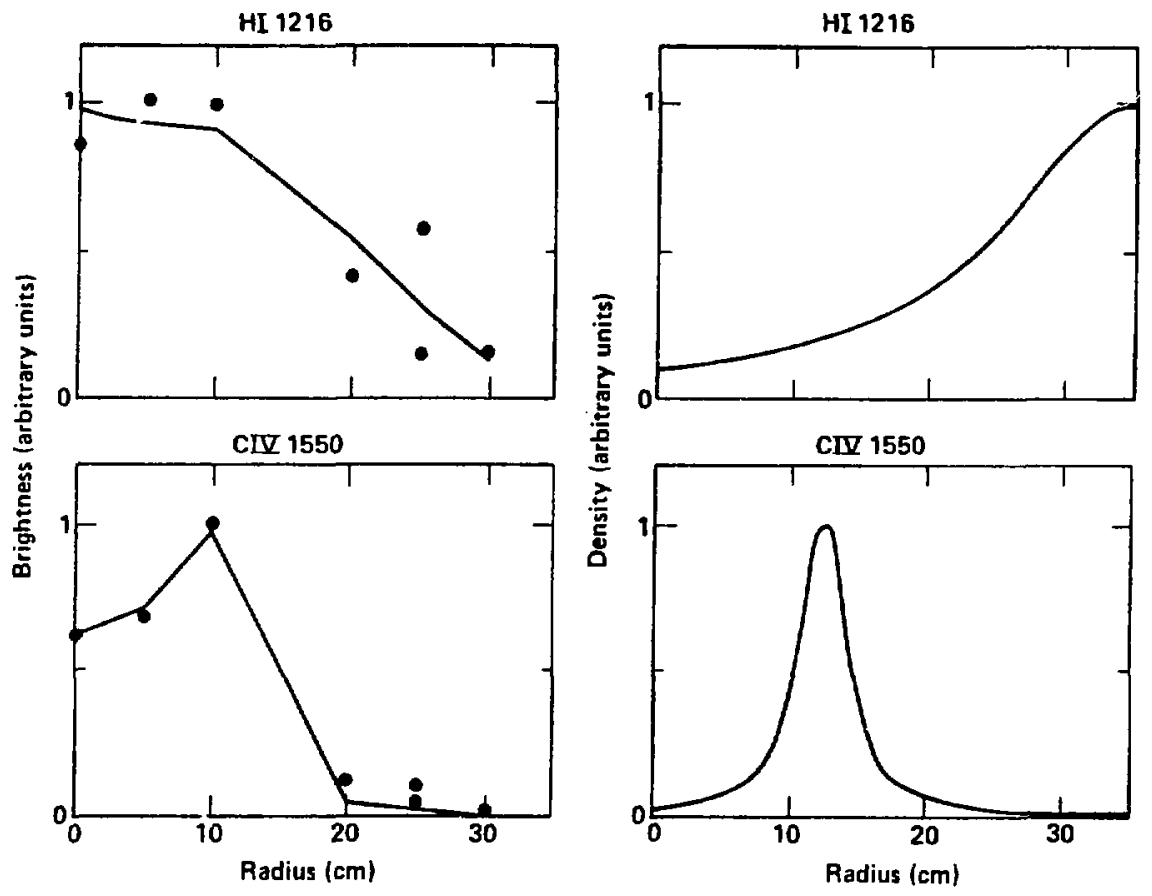

Fig. IV.8 Similar to Fig. IV.6 \& 7 except that now the profiles of neutraldeuterium and C IV emissions are shown. 

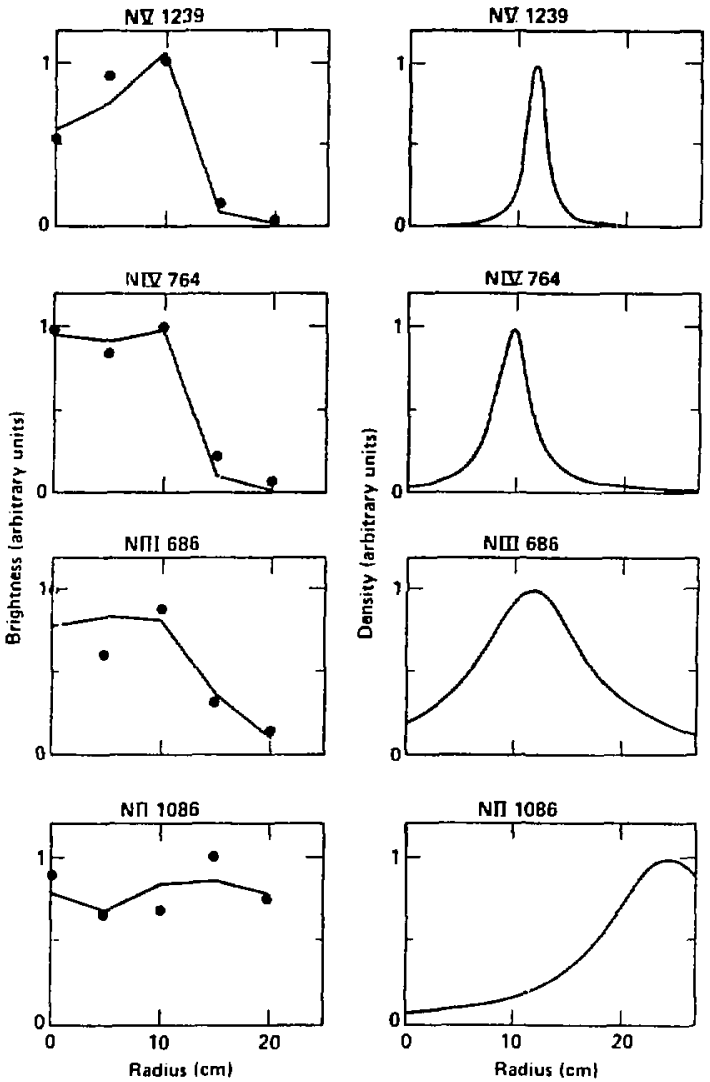

Fig. IV.9 This figure shows nitrogen ions profiles which correspond to the case of $\mathrm{N}$-beam injection shots. 


$$
B_{i}(x) \sim \int\left\langle o v>_{i e x} n_{\theta} n_{i 0} /\left(1+\left(r-r_{i o}\right)^{2} / D_{i}^{2}\right) d l\right.
$$

Note that $<\sigma v>_{\text {iex }}$ was temperature and hence radial dependent; and $x$, the chord position was related to $r$ and $I$ by

$$
r^{2}=1^{2}+x^{2}
$$

For a given $x$ and a certain set of $n_{i 0}, r_{i 0}$, and $D_{i}$, we could obtain the value of the $B_{i}(x)$ from Eq. (IV.12) by numerical integration. This enabled us to perform a least square fitting to the directly measured data points of brightness with $n_{i 0}, r_{i o}$, and $D_{i}$ as fitting parameters. Fig. IV.6-9 show the ion density profiles obtained in this way (the corresponding brightness values and data points are also shown). We can see from the figures that the distributions of the ions of different ionization states are different. It should be mentioned that the fitting has been performed to determine the sensivitity of the fitted profiles to yariations in electron temperature and density. We found that the fitted profiles were fairly insensitive to a reasonable variation $(<30 \%)$ of $n_{\theta}$ and $T_{e}$. Note that the nitrogen radial profiles obtained during the $\mathrm{N}$-beam-injection experiment were quite different from those obtained from the usual run. (We assumed that in both cases, the nitrogen was injected by neutral beams.) in the former case, the NV density profile peaked quite sharply at about $10 \mathrm{~cm}$ while in the latter case, it peaked at the center and extended smoothly to the edge. This is partly because in contrast to the usual (deuterium beam) case, during the nitrogen beam-injection experiment, the neutral beams were aiming along chords which were $10 \mathrm{~cm}$ from the central axis. The difference may also be due to uncertainly in the constancy of the shots during shot-by-shot scans.

In order to obtain a perspective for these experimental density profiles, we compared them with the profiles predicted by a commonly used one dimensional 
transport computer model. The details of this model are presented in Appendix C. In this model,

$$
\frac{\partial n_{q}}{\partial t}+\frac{1}{r} \frac{\partial}{\partial r}\left(r r_{q}\right)=-\left(I_{q}+R_{q}\right) n_{q}+l_{q-1} n_{q-1}+R_{q+1} n_{q+1}-n_{q} / \tau_{/ /}+S_{q}
$$

Here, $q$ is the ion charge, $n_{q}$ is the density of charge state $q, r_{q}$ is the radial flux for charge state $q_{1} R_{q}$ is the total recombination rate, $l_{q}$ is the total ionization rate. $\tau_{/ /}^{-1}$ denotes the parallel loss rate, and $S_{q}$ is a volume source for $q=+1$ resulting from attenuation of beam-injected neutrals or from edge neutral influx. The rate coefticients depend strongly on the electron temperature $\mathrm{T}_{\theta}$ which varies widely over space. The coupled equations of (IV.14) can be solved numerically by the finite difference techniques"116,117. For simplicity, we employ a general diffusive/ convective model to represent the flux.

i.e. $\tau_{q}(r, t)=-D_{q}(r)\left(\partial n_{q} / \partial r\right)+V_{q}(r) n_{q}(r, t)$

where the diffusive coefficient $D_{q}(r)$ and convective velocity $V_{q}(r)$ are assumed to be independent of time and charge state. This model has been used in transport studies of low- and high-z impurities $\$ 17-118$, and for studies of the plasma density buildup on tokamaiks ${ }^{119}$. Since this is an empirical procedure, we consider a simple case of (IV.15),

$$
I_{q}(r, t)=-D\left(\partial n_{q} / \partial r\right)+(r / a) V_{0} n_{q}
$$

wher $\theta a$ is the limiter radius, and $D$ and $V_{0}$ art independent of radius. 
Because we have obtained simple analytic expressions for the time evolution of the impurities in the last section, here we only consider the numerical solutions for the steady state case (i.e. $\partial n_{q} / \partial t=0$ ). We choose the boundary conditions to be $I_{q}(0)=0$ (this has to be true if $I_{q}$ has azimuthal symmetry) and $n_{q}(a)=0$ for all $q$ except for $q=0$ ( the non-zero density of the neutrals at the boundary represents the reflux of the impurities or an impurity flux from the wall). Now we can adjust $D$ and $V_{0}$ to produce simulated profiles which are in good agreement with experimental fitted profiles. It should be noted that in (IV.16), a positive $V_{0}$ (convective velocity at the limiter radius) represents an outward flux while a negative $V_{0}$ represents an inward flux. Profiles for different sets of $D$ and $V_{0}$ appropriate for our experimental data are presented in figures $\mathrm{N} \cdot 10-13$. The profiles of the +1 source term are also shown. For the oxygen and nitrogen profiles of the usual shots, we assumed that the source was from attenuation of the beam particles which were injected along the central chords across the plasma (more quantitative discussions of the source term are presented in Appendix $\mathrm{C}$ ); the effect of the reflux has been considered by including a non-zero density of the impurity neutrals at the edge (at $r=0$ ). For the carbon profiles, the source was the neutrals from the wall. The $T_{e o}$ used is $70 \mathrm{eV}$ which is from a typical shot In the case of $\mathrm{N}$-beam-injection experiment, the source $\mathrm{N}^{+1}$ was from the attenuation of the neutrals along chords which are $10 \mathrm{~cm}$ from the central axis; a reflux term is also included. The ELA diagnc stics and line ratios of different impurity ionization states show that the $\mathrm{T}_{\mathrm{eo}}$ was lower in this case; we have used a value of $50 \mathrm{eV}$ for $\mathrm{T}_{\mathrm{eo}}$ in the simulation. In both cases (the usual shots and $\mathrm{N}$-beam-injection shots) we assumed $\tau_{/ /}=5 \mathrm{~ms}$. We found that in the case of the usual run, the values of $D$ and $V_{0}$ that can describe the experimental profiles best were in the range 2000 $\pm 1000 \mathrm{~cm}^{2} / \mathrm{s}$ and $-2000 \pm 500 \mathrm{~cm} / \mathrm{s}$ respectively. The negative $V_{0}$ implies that we have an inward convective term. These values are fairly close to those 


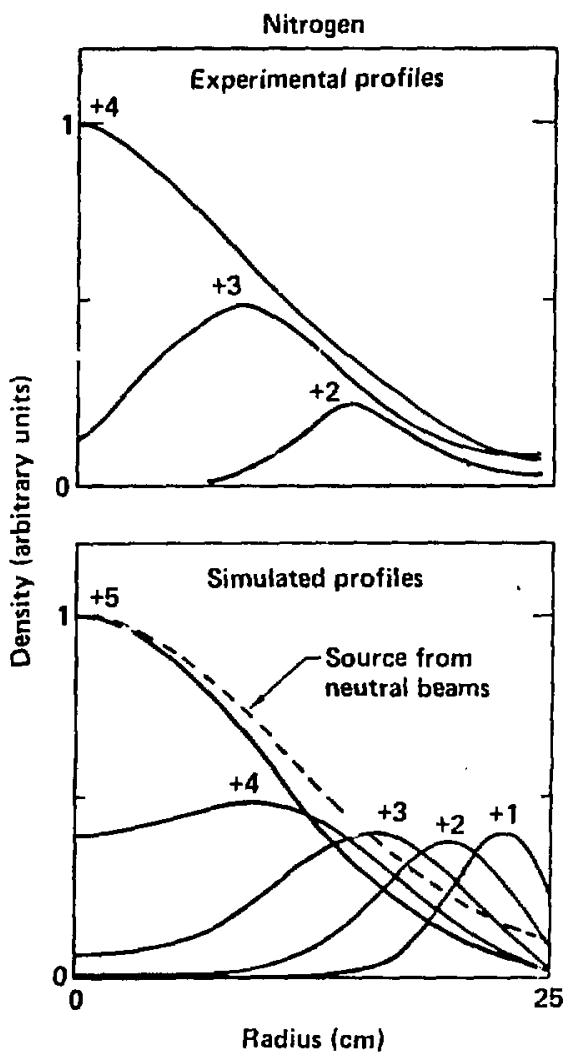

Fig. IV.10 The upper half of the figure shows the experimental fitted density profiles of different nitrogen ionization states plotted on the same scale. The lower half shows the simulaled density profiles which are close to the experimental profiles. The dotted line shows the profile of the +1 source which was produced from the attenuation of neutral beam particles. The central temperature used is $70 \mathrm{eV}$, and the parallel confinement time is $5 \mathrm{~ms}$. 


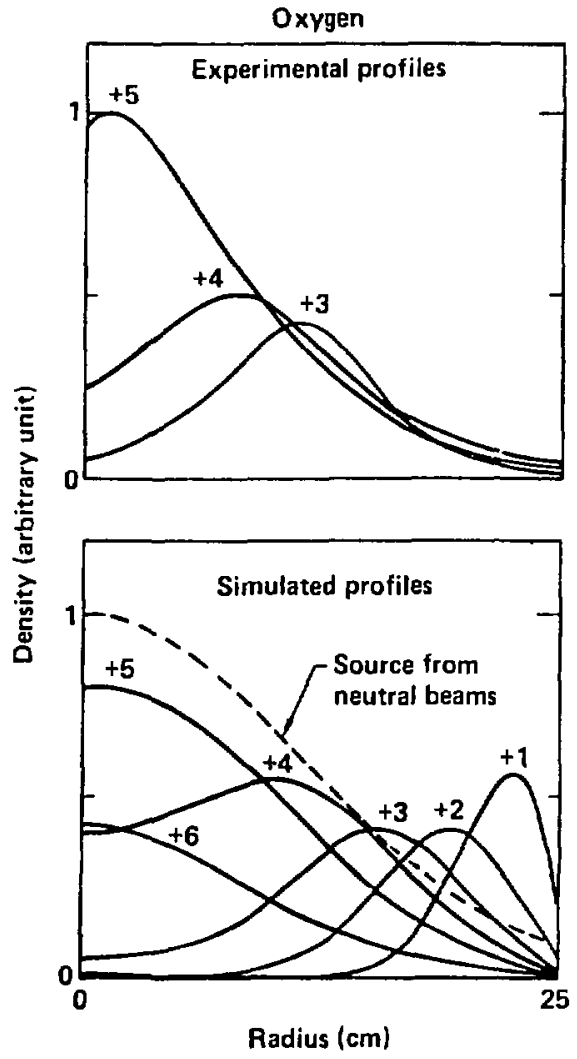

Fig. IV.11 Similar to Fig. IV.10 except that this figure describes the oxygen impurity. 


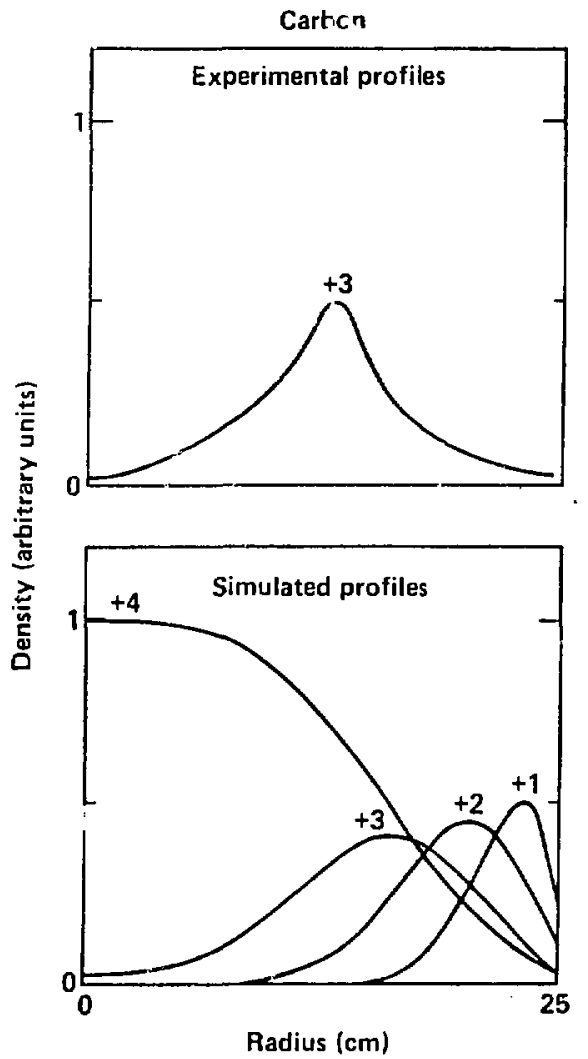

Fig. IV.12 Similar to Fig. IV.10 \& 11 except that this figure describes the carbon impurity and no neutral beam source has been assumed. 


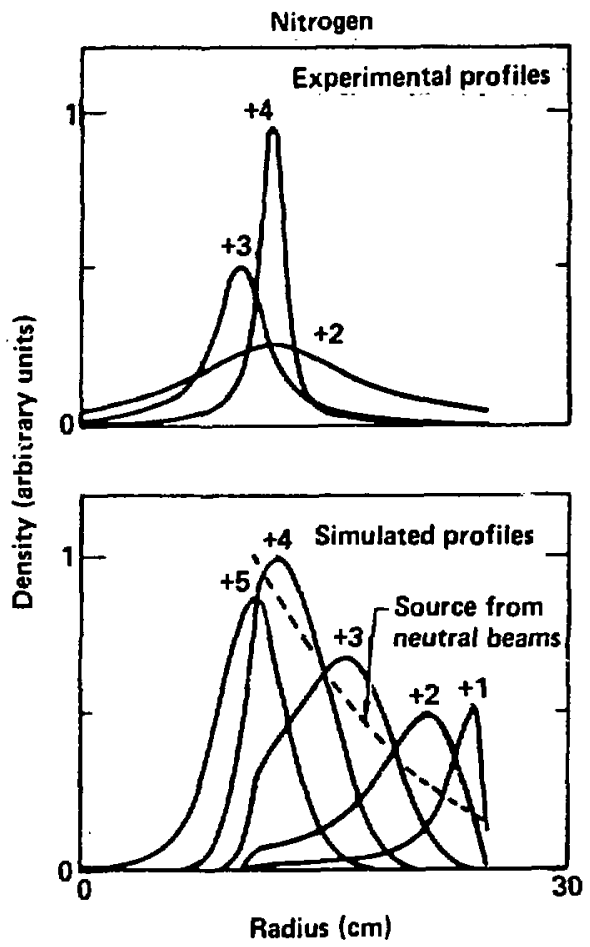

Fig. IV.13 This figure corresponds to $\mathrm{N}$-beam injection shots. The neutral beams were aiming along chords which were $10 \mathrm{~cm}$ away from the axis. The central temperature was $50 \mathrm{eV}$. 
obtained in tokamak devices ${ }^{120-123}$. On the other hand, during the $\mathrm{N}$-beam injection experiment, the magnitudes of $D$ and $V_{0}$ obtained in this way are much smaller. The range of the values of $D$ for this case is 100 to $500 \mathrm{~cm}^{2} / \mathrm{s}$, and that of $V_{0}$ is -500 to $-100 \mathrm{~cm} / \mathrm{s}$. This implies that the transport mechanisms in the two cases were different.

The possibility that this difference is in fact due to the uncertainty of the reproducibility of the $\mathrm{N}$-beam-injection shots should not be ruled out. Future work should clarify this discrepancy.

In this chapter, we have estimated the global confinement time of beam injected nitrogen ions from the concentration of the ions in the plasma as well as from the decay time histories from the ion emissions. The value was found to be between 1 and $10 \mathrm{~ms}$. We also studied the radial transport of impurities in TMX-U with a one dimensional diffusive/convective transport model. For the normal shots, we found that the values of diffusive constant $D$ and convective $V_{0}$ which best describes the data are close to those obtained in tokamak devices, but quite different from those obtained during the nitrogen-beam-injection experiment. 


\section{Chapter $V$ Development of possible new $n_{e}$ and $T_{e}$ diagnostics}

\section{V.1 Introduction}

In the previous few chapters, we have discussed how we use EUV spectroscopy to estimate the concentrations of impurities and the associated radiative power losses, to study thermal barrier filling and transport phenomena. The same spectroscopic data can also be used for other plasma diagnostics. For example, we can determine the ion temperature from Doppler broadening ${ }^{124-125}$ or from forbidden to allowed line ratios ${ }^{126}$; determine the magnetic field from Zeeman splitting ${ }^{127-128}$; estimate the electron density from Stark broadening ${ }^{129-130}$. Here, we discuss how we use the time-resolviny 1024-channel spectrograph to determine $T_{e}$ and $n_{e}$ in TMX-U by considering the ratio of an appropriate pair of lines emitted by the same ionization state. In general, spectroscopic determinations of $n_{e}$ and $T_{e}$ require repeated constant shots to allow a radial scan to determine where the emitting ions are located. If impurities under study do not exist in the plasma, impurity injection is required. Despite these requirements, spectroscopic methods have special advantages over the traditional techniques. They tend to cover a much wider dynamic range and have relatively high time resolution. Also, they are relatively simple and inexpensive diagnostics. Thus on TMX-U where in many cases, the density and temperature were very low and outside the design parameters of the Thomson scattering apparatus, spectroscopic techniques were useful for estimating $T_{e}$. Spectroscopic techniques are especially useful for monitoring changes in the plasma parameters. Uniortunately, the models used to relate the data to plasma parameters are based on theoretical atomic models with very few experimental checks or very often the models tend to rely on a 
combination of theory and self consistency arguments from astrophysical data.

Therefore, we first check the atomic computations against some of our data obtained under known running conditions. Only after we made such a comparison, could we apply the atomic computations to predict $n_{e}$ and $T_{e}$ with confidence.

\section{V.2 Experimental Evidence for The Effect of Resonance Structures on Electron Excitation Rates for Si III 131}

Line ratios of Si III have been useful to predict electron densities and temperatures in the transition region of the sun. However, utilizing different atomic theories for prediction can lead to an order of magnitude difference in electron density ${ }^{132}$. In this section and the next we show that the resonance structures in the collision strengths play an important role in the computations of line ratios for low $\mathrm{Z}$ ions. Omitting them would lead to large discrepancies in the computation.

Several papers published by the group at the Queens University of Bellast have pointed out that the complicated resonance structures play an important role in collision strengths at low energies ${ }^{133-136}$. The collision rates obtained by these authors for low $\mathrm{Z}$ ions isoelectronic with $\mathrm{Be} \mathrm{I}$ and $\mathrm{Mg} I$ using the R-matrix method ${ }^{137}$ were significantly higher than those obtained using distorted wave computations. In a recent paper ${ }^{132}$, Dufton et al. used the R-matrix results in a 10 -level model to obtain Si III line ratios to predict electron density and temperature of the solar transition region. They also compared their computed values with those obtained by Nicolas et al. ${ }^{138}$ which used the distorted wave method; the R-matrix rate for the $3 s^{2}{ }^{1} S-3 s 3 p{ }^{3} P$ transition was a factor of 4 larger than the one obtained by the distorted wave method. Dufton et al. noted that this difference was mainly due to the complicated resonance structures in collision strength at low energy; these 
were not included in the distorted-wave calculations. In this section, we show that the experimental data agrees with the R-matrix rather than distorted wave predications indicating that the resonance structures are important in computing electron excitation rates for Si III ${ }^{131}$.

\section{V.2.1 Experimental Data}

Silicon spectra were recorded by the time-resolving spectrograph in the $1100-1400 \AA$ (grating F) range for the central cell plasma of TMX-U; the resolution of this grating is about $1 \AA$. Silicon atoms were introduced into the plasma when the quartz window of the ECRH gyrotron was damaged by high voltage arcs. These phenomena were not common, but we were able to obtain several good shots. Based on the radial profiles of $\mathrm{C}, \mathrm{N}$ and $\mathrm{O}$ discussed in Chapter IV and the low ionization potential of Si III, we concluded that the Si III ions were located near the edge $(r>15 \mathrm{~cm})$ of the plasma. In these shots, electron densities were estimated to be $4 \times 10^{11} \mathrm{~cm}^{-3}$ on the axis of the plasma and about $1 \times 10^{11} \mathrm{~cm}^{-3}$ at the plasma edge. From the Thomson scattering and Langmuir probe data, we concluded that the $T_{e}$ at the edge in these shots weres $10 \mathrm{eV}$. (Note that the conclusions of this section do not depend sensitively on the value of $T_{\theta}$.)

The Si III lines of interest were the $3 s 3 p{ }^{3} P(6.6 \mathrm{eV})-3 p^{2} 3 p(16.1 \mathrm{eV})$ and the $3 s 3 p{ }^{3} P(10.3 e V)-3 s 4 s^{1} S(19.7 \mathrm{eV})$ transitions. (The values in parentheses are the energies above the $3 s^{2}{ }^{1} \mathrm{~S}$ ground state.) These lines are concentrated within a $15 \AA$ range interval making their relative brightness measurements quite reliable. They are generally unblended. The transition at $\lambda 1303.3$ could be contaminated by Si II $3 s^{2} 3 p-3 s 3 p^{2}$ at $\lambda 1304.4$. By examing other Si Il lines, we concluded that the contamination was small. Fig. V. 1 presents the experimental spectrum between 1290 and $1315 \AA$ and $a$ fitted spectrum. We obtained the brightness estimates by using a fitting 

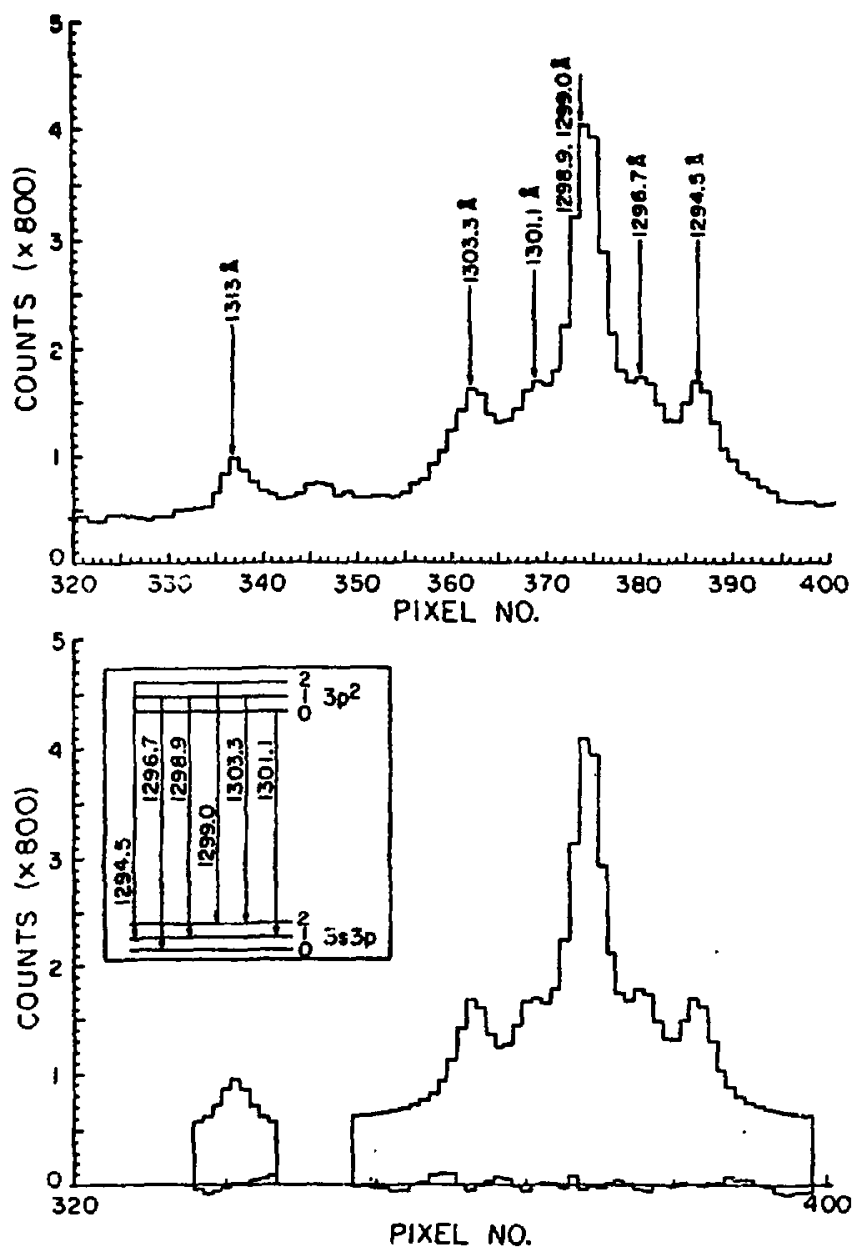

Flg. V.1 The upper hall of the figure (a) shows a portion of the Si lil spectrum between 1290 and $1315 \mathrm{~A}$. The lower half (b) shows the ritted spectrum" (see explanation in text). At the bottom of the figure, the difference between (a) and (b) is shown on the same scale. The inset shows the transition between the wo excited states, $3 s 3 p p^{3}$ and $3 p^{2} 3 p$. 
procedure described in the following paragraph.

The hydrogen $L y \propto$ line at $1216 \AA$ has a high signal to noise ratio and is unblended. Therefore, we used it as a model of the line shape of the emission lines. It was fitted with a profile described by the convolution of a rectangular and Lorentzian shape. The position of the line peaks were taken as known as the wavelength had been obtained independently. The "cluster" of lines around $1300 \mathrm{~A}$ was then expressed as the linear combination of the six Si III lines each having a profile of the type mentioned above. Note that some of the six lines are related to each other by branching ratio which are non-adjustable parameters, resulting in only three independent lines. We then used a linear least square fitting to determine the coefficients of the linear expansion, which represented the line brightnesses. The resulting uncertainties in the relative line brightness estimates were thus indicators of the quality of the fit.

\section{V.2.2 Discussions of Results}

We examined two line ratios $R_{2}$ and $R_{3}$ defined by Dufton et al. ${ }^{132}$ (see Table V.1 for definition). Under our experimental conditions, we could not use the temperature sensitive ratio $R_{1}$, because of the blend of the line $1892 A$ with the third order of the bright intrinsic O V $630 \mathrm{~A}$. In order to cross check the temperature estimates obtained with $R_{2}$, we also examined ratio $R^{*}$ between the lines at 1294 and $1313 \AA$. The values based on Dufton et al. ${ }^{132}$ as well as values based on Nicolas et al. ${ }^{138}$ appropriate to the density and temperature conditions of the plasma are listed.

The ratio $R_{3}(=\lambda 1301 / \lambda 1296)$ is sensitive to density because the upper level of $\lambda 1301,3 p^{2}{ }^{3} P_{0}$ is mainly populated by electron impact excitation from the $3 s 3 p{ }^{3} P_{1}$ metastable lower level. On the other hand, the upper level of $\lambda 1296,3 p^{2} 3 P_{1}$ is populated from all three $3 s 3 p{ }^{3} P_{0,1,2}$ metastable 


\section{Table V.1}

\section{Comparison between Theory and Experiment for Si III Ratios}

Line Ratio

$$
\begin{array}{ccc}
\begin{array}{c}
\text { Theory }\left(\mathrm{n}_{\mathrm{e}}=1 \times 10^{11} \mathrm{~cm}^{-3}\right) \\
\text { Dufton et al. } \\
\text { (R-matrix) }
\end{array} & \begin{array}{c}
\text { Nicolas } \\
\text { (Distorted wave) }
\end{array} \\
\mathrm{T}_{\mathrm{e}}(\mathrm{eV})=3 \quad 5 & 8 & 5
\end{array}
$$

$R_{3}=\frac{\|(1301 \AA)}{I(1296 \AA)}$

$R_{2}=\frac{\mid(1301 \AA)}{I(1313 \AA)}$

$R^{*}=\frac{I(1294 \AA)}{I(1313 \AA)}$

6.2

3.3

2.1 
levels. Among the three metastable levels, $3 s 3 p{ }^{3} P_{0,1,2}$, only the $3 s 3 p{ }^{3} P_{1}$ state has a large radiative decay rate to the ground state. Its population is therefore much lower than a Boltzmann population relative to the ${ }^{3} F_{0,2}$ leve's at low electron densities. It will be increasing towards the Boltzmann population as the collisional dexcitation rate (which is proportional to the electron density) increases and approaches the radiative decay rate of $3 s 3 p{ }^{3} P_{1}$ to the ground state. Therefore, the ratio $R_{3}(=\lambda 1301 / \lambda 1296)$ is sensitive to derisity change.

Figure V.2 compares the experimental value of $R_{3}$ with the two theoretical calculations of this density sensitive line ratio. (The ratio $R_{3}$ is rather insensitive to the temperature as it involves two transitions within the triplet state and the excitation energies of the upper levels are very close. Howover, as discussed in the following paragraphs, $R_{2}$ and $R^{*}$ imply $6-8 \mathrm{eV}$ for the plasma, a reasonable value near the plasma edge.) The experimental values agree quite well with the prediction of the R-matrix computation but differ from the prediction of the distorted wave by nearly a factor of two which is much larger than our experimental uncertainties.

As mentioned earlier in this section, the difference in the ratio $R_{3}$ predicted from the two sets of calculations is mainly due to the enhancement in the $2 s^{2}{ }^{1} s-3 s 3 p{ }^{3} p$ excitation rate resulting from the resonance effects. The line ratio $R_{3}$ is sensitive to the relative rates between the radiative decay and electron impact de-excitation of the $3 s 3 p{ }^{3} P_{1}$ state. Therefore, for a given value of $R_{3}$, the density predicted by Dufton et al.'s data would be lower than trat predicted by Nicolas' data. Our result reflects the difference between these two sets of calculations.

We also evaluated the ratios $R_{2}$ and $R^{*}$ to set the approximate temperature range for evaluating $R_{3}$, lo check for consistency of the values predicted by $R_{2}$ versus $R^{*}$, and to compare the temperature predicted by $R_{2}$ and $R^{*}$ with the estimated temperature near the edge of the plasma. In our case, the 


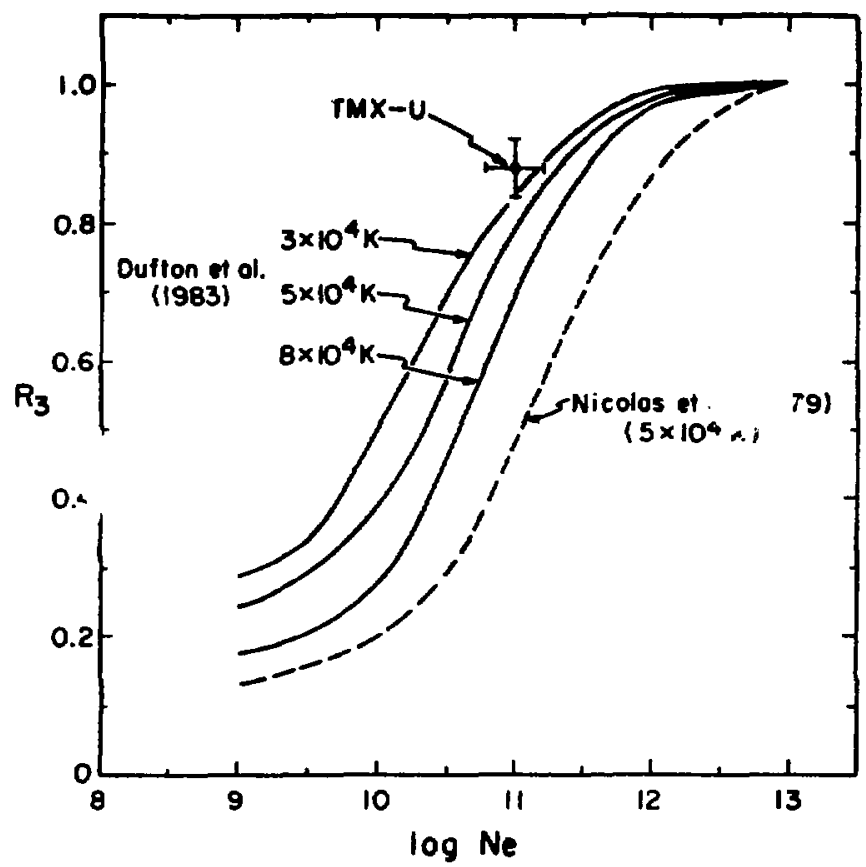

Fig. V.2 Comparison of theoretical and experimental values for the ratio $R_{3}$. The solid curves are from Dutton et al ${ }^{132}$. lor three electron temperatures. The dashed curve is from Nicolas of al ${ }^{138}$. 
experimental values compared to Dufton et al. imply temperatures of $6 \mathrm{eV}$ from $R_{2}$ and $8 \mathrm{eV}$ from $R^{*}$, which are in agreement within the range of the uncertainties. (This is to be compared with an ionization equilibrium temperature of $3.2 \mathrm{eV}$ and is consistent with the fact that the radial transport has shifted the particles towards regions with temperatures higher than the equilibrium value ${ }^{59}$ ). These values are also consistent with the values estimated by Thomson scattering and Langmuir probe data discussed above. Nicolas et al. would imply higher temperatures.

In conclusion, experimental measurements of $\mathrm{Si}$ III line ratios have cenfirmed the importance of the resonance structures in calculating the colision strengths at low energy. The new results significantly improve the reliability of the estimates of the electron density and temperature of the solar corona and flares based on Si III line ratios. The Si III electron density and temperature diagnostics are appropriate to the plasma conditions of TMX-U running at the thermal barrier mode. If a proper impurity injection system is installed, this would give us an alternative to measure $n_{e}$ and $T_{e}$ in $T M X-U$.

\section{\.3 $\mathrm{n}_{\mathrm{e}}$ Diagnostics Using Be I-like Ions $\Delta \mathrm{n}=0(2-2)$ Emissions}

Lines originating from $\Delta n=0(2-2)$ transition of $\mathrm{Be}$ l-like $\mathrm{C}, \mathrm{N}, \mathrm{O}$ and Ne ions have been used extensively for $n_{e}$ and $T_{\theta}$ diagnostics of astrophysical plasmas ${ }^{139-141}$. The line ratios of $2 s 2 p{ }^{3} p-2 p^{2}{ }^{3} p$ transitions to $2 s^{2}{ }^{1} s_{0}$ - $2 s 2 p{ }^{1} P_{1}$ transitions of these ions are sensitive to electron density in the range $10^{9} \cdot 10^{13} \mathrm{~cm}^{-3}$. This is because the $2 s 2 p{ }^{1} \mathrm{P}_{1}$ level is populated mainly by electron impact excitation from the ground state, $2 s^{2}{ }^{1} \mathrm{~S}$, while $2 \mathrm{p}^{2}$ ${ }^{3} P$ levels are populated from the $252 p{ }^{3} P$ levels. The reliability of $n_{\theta}$ or $T_{\theta}$ 
diagnostics of astrophysical plasma from these line ratios rely heavily on the atomic models. Different models often do not agree among themselves or are not consistent with the observed data. For example, in the analysis of the $\mathrm{OV} \Delta \mathrm{n}=$ O(2-2) line ratios; the predictions of density dependence of Malinovskey 142 was quite different from those of Dufton et al. ${ }^{140}$. Moreover, both of them disagreed with observations ${ }^{143}$.

In order to test the atomic models, we conducted impurity experiments in the TEXT tokamak during the period of $9 / 1984$ - 12/1984 using the same normal incidence 1024-channel spectrograph that we use in TMX-U and a grazing incidence time resolving spectrograph (GRITS) ${ }^{144-145}$. All the Be H-like ions emissions discussed in this section were recorded by the survey grating ( $300-1800 \AA$ ) of the rormal incidence spectrograph. The GRITS was used to monitor the positions of the emitting ions of interest by radial scans. The electron temperature and density of the plasma of TEXT were measured by Thomson scattering, far infared (FIR) interferometry and Langmuir probes ${ }^{146}$. The details of this experiment are discussed in reference 147 . The characteistics of the TEXT tokamak are shown in figure V.3. The locations of the Be l-like ions studied here are shown in figlure V.4. The $n_{e}$ and $T_{e}$ of a typical TEXT tokamak plasma are also shown in this figure.

Because all the lines concerned were always recorded by the survey grating of the 1024-channel spectrograph, we were able to calculate the brightness of both the resonance $2 s^{2}$ is $-2 s 2 p{ }^{1} P$ line and metastable $2 s 2 p{ }^{3} P-2 p^{2} 3 p$ line in the same plasma shot. In the survey spectra, the resonance and the metastable lines of a given element were always close to each other. Therefore, the uncertainty arising from the calibration of the relative sensitivity of the detector was small. Moreover, the signal to noise ratios of both the resonance and metastable lines were always large. Both of these reasons imply that the experimental line ratios discussed here are quite reliable. We have examined Be H-like C, O, F and Ne.

Both carbon and oxygen were intrinsic in the TEXT tokamak. The other two elements considered, $\mathrm{F}$ and $\mathrm{Ne}$ were not intrinsic and we introduced them into 


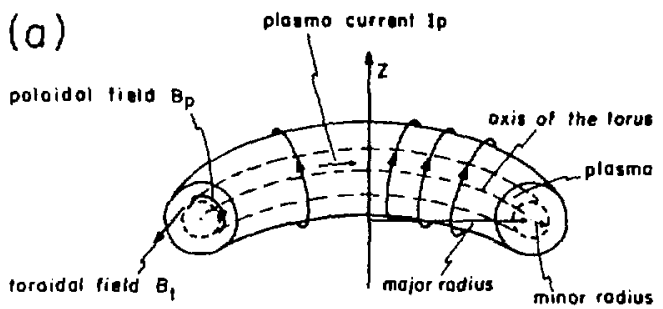

(b)

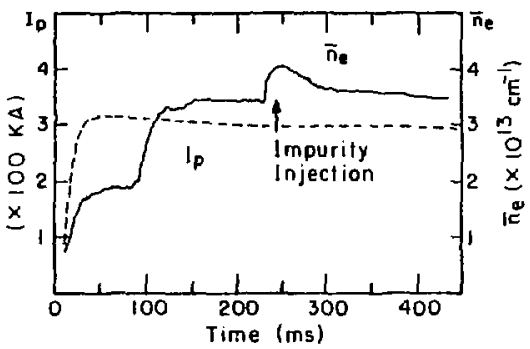

(c)

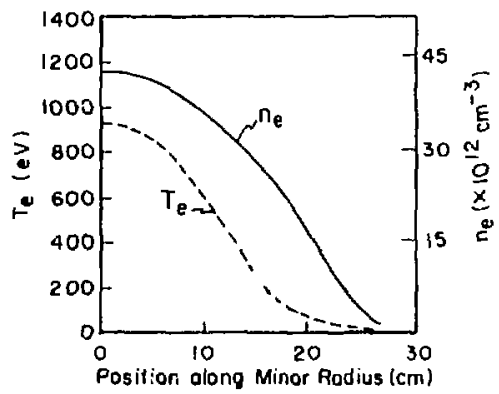

Fig. V.3 Characteristics of TEXT tokamak. a) configuration of TEXT tokamak, b) time historeis of plasma current $I_{p}$ (dashed line) and line average density $n_{\theta}$ (solid line) of a nominal discharge with impurity injection, and $c$ ) radial profiles of electron temperature (dashed line) and electron density (solid line) of a standard TEXT discharge $/_{A}=300 \mathrm{kA}, n_{\theta}=3 \times 10^{13} \mathrm{~cm}^{-3}$, $\mathrm{B}_{\mathrm{T}}=28 \mathrm{kG}$. 


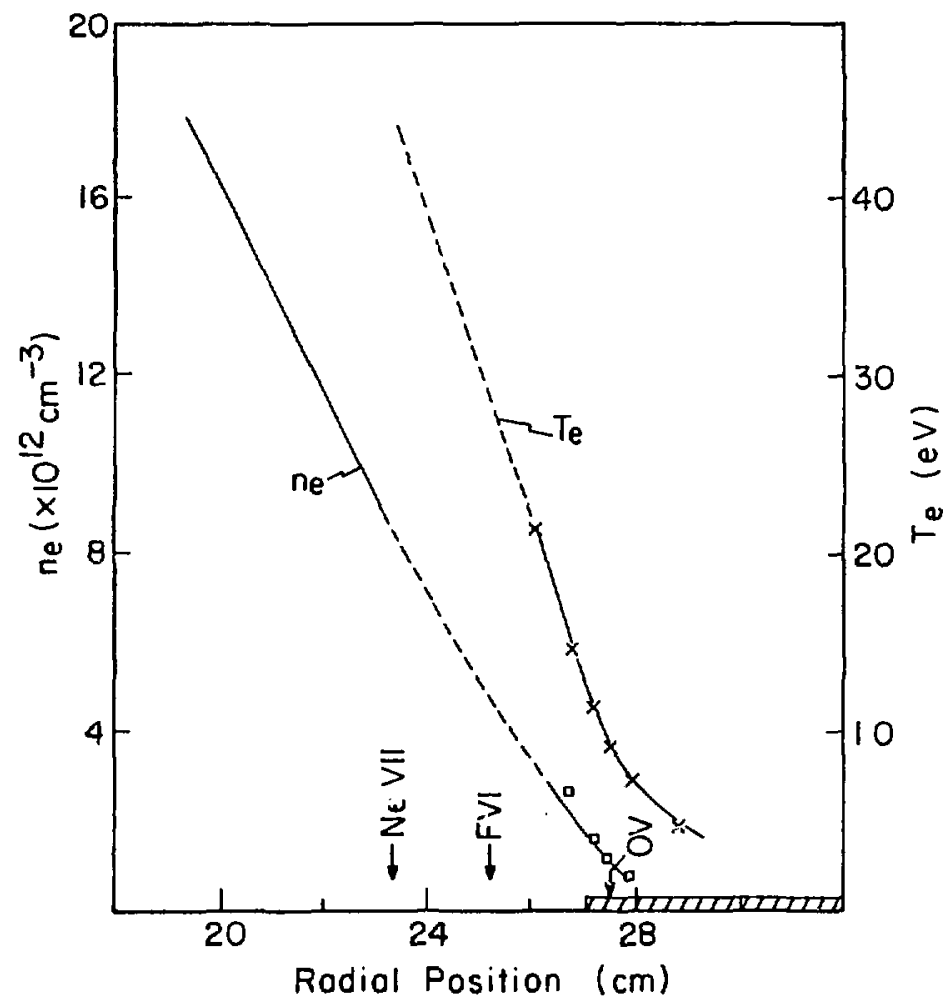

Fig. V.4 Electron density (lower curve) and temperature (upper curve) near the edge of the TEXT tokamak plasma. The positions of the emitting shells of O V, FVI and Ne VII ions obtained on a shot by shot basis are shown. The shaded area represents the position of the limiter. 
the machine either by a laser-ablation technique ${ }^{148}$ or a gas puff through a fast valve. We introduced fluorine into the tokamak plasma rather than nitrogen because the density dependence of the $F$ VI line ratio is much more pronounced in the range of interest than that of NIV (see figure V.5).

A simplified Grotrian diagram for the $n=2$ levels and the interested transitions of the Be l-like ions is shown in figure V.5a. In this section, we only consider the line ratios

$$
R=\frac{1\left(2 s 2 p^{3} p-2 p^{23} p\right)}{\mid\left(2 s^{21} S_{0}-2 s 2 p^{1} P_{1}\right)}
$$

We compared our experimental results with two different sets of computations. The first set $140,141,149$ used the R-matrix method discussed in section V.2. As mentioned in the last section; this method has included the resonance structures in the computations. In this set of computations, the atomic radiative transition rates were based on the results of Nussbaumer and Storey ${ }^{150}$ and the proton excitation rates were based on the results of Doyle et al. ${ }^{151}$. Fig. V.5b shows the theoretical values of $R$ from $\mathrm{C}$ III to $\mathrm{Si}$ III based on this method by the Belfast group $140,141,151-153$. The second set of computations was performed by Bhatia ${ }^{147}$ for $C$ III and $O V$. It differed from the first set mainly in three ways : a) the distorted wave approximation was used to calculate the collision strengths to obtain the electron impact excitation rate. Specifically, the effects of the resonances were not included. b) population of lower levels from higher levels through cascading were included. c) the semi-classical theory of Seaton ${ }^{154}$ was employed to compute the proton excitation rate. The line ratios obtained by the two sets of computations disagreed by more than a factor of two for the value of $R$ in $C I^{\prime}$ at the ionization equilibrium temperature and a density of $1 \times 10^{12} \mathrm{~cm}^{-3}$. The disagreement is 

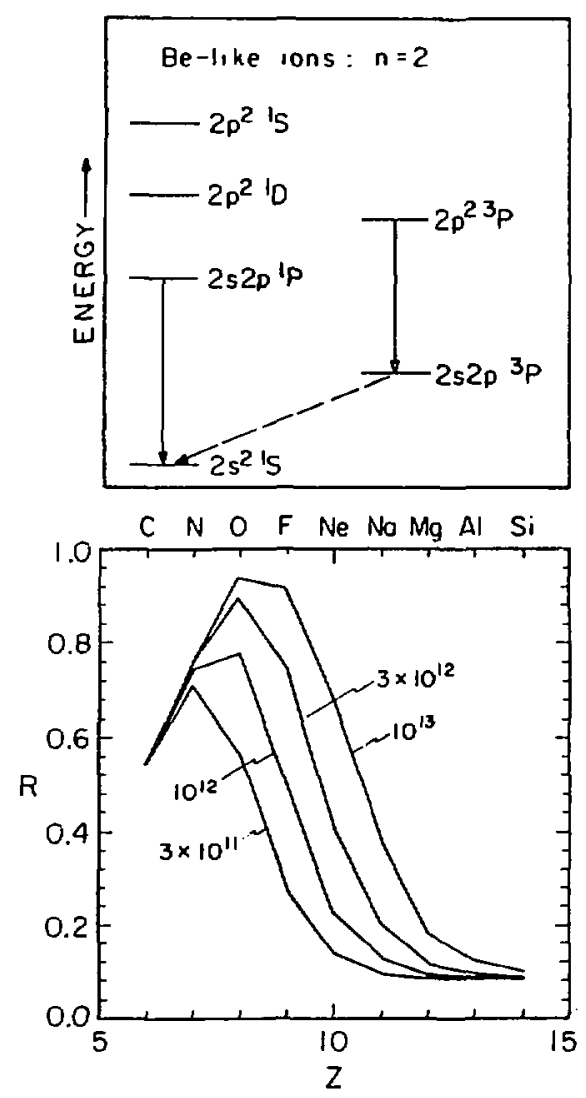

Fig. V.5 a) Simplified Grotian diagram of the $n=2$ levels of Be l-like ions showing the transitions used in this work. b) Theoretical values of the ratio $R$ from C III to Si XII at temperatures near their corresponding ionization equilibrium temperatures for four different densities. 
expected to decrease with increasing nuclear charge $Z$. As expected, in the $O \mathrm{~V}$ case, the difference was much less $(\leqslant 30 \%$ in most cases). It has been pointed out in reference 140 , the discrepancy arose principally from the complicated resonance structures in collision strength, this having not been included in the distorted wave method used in the second set of calculations.

For the $\mathrm{C}$ III case (where the discrepancy was pronounced), our experimental value was much closer to the values predicted by the first set of computations ( in which the resonance effects were included by using the R-matrix method) This is a confirmation to our conclusions in section V.2 that resonance structures are important in calculating the collision strengths at low energy. Comparison of the experimental and theoretical R-matrix results for the $\mathrm{A}$ values of $\mathrm{C} I \mathrm{Il}, \mathrm{OV}, \mathrm{FVI}$, and $\mathrm{Ne} \mathrm{Vll}$ are summarized in Table V.2 and presented in Fig. V.6. We can see from this table and figure that the experimental and R-matrix results agree quite well. Therefore, the Be 1-like line ratios of $i$ computed by the $R$-matrix method are reliable and serve as accurate electron density diagnostics.

After finding out that the computed $\mathrm{R}$ values by the Belfast group are reliable, we can use their computed curves along with our measured ratios as an $\mathrm{n}_{e}$ diagnostic for the TMX-U plasma. We discuss in the following paragraph how we used the intrinsic $N ! V$ and $O V$ line ratios to measure $n_{e}$ in TMX-U. The $R$ ratio of $\mathrm{C}$ III does not have density dependence and was not used.

In applying this method to estimate $n_{e}$, we need to know the $T_{e}$ of the plasma a priori. However, the computed curves of low Z Be-like ions such as N N and $O V$ are rather insensitive to a change in $T_{e}$. Even though the $T_{e}$ changed by $a$ factor of two, the predicted $n_{e}$ from the line ratios would not change by more than $50 \%$. We also need to know where the emitting ions are located. We have discussed the ion density profiles of $\mathrm{N}$ and $\mathrm{O}$ in the last chapter. We assume in TMX-U that $O V$ is at about $8 \mathrm{~cm}$ from the central axis, and $N I V$ is about $10 \mathrm{~cm}$ from the axis. The electron density is described by a Gaussian with 1/e radius of $20 \mathrm{~cm}$. Deviations from these two assumptions can cause about $50 \%$ error. We selected several shots in each of which the intrinsic ions (N IV or O V) 


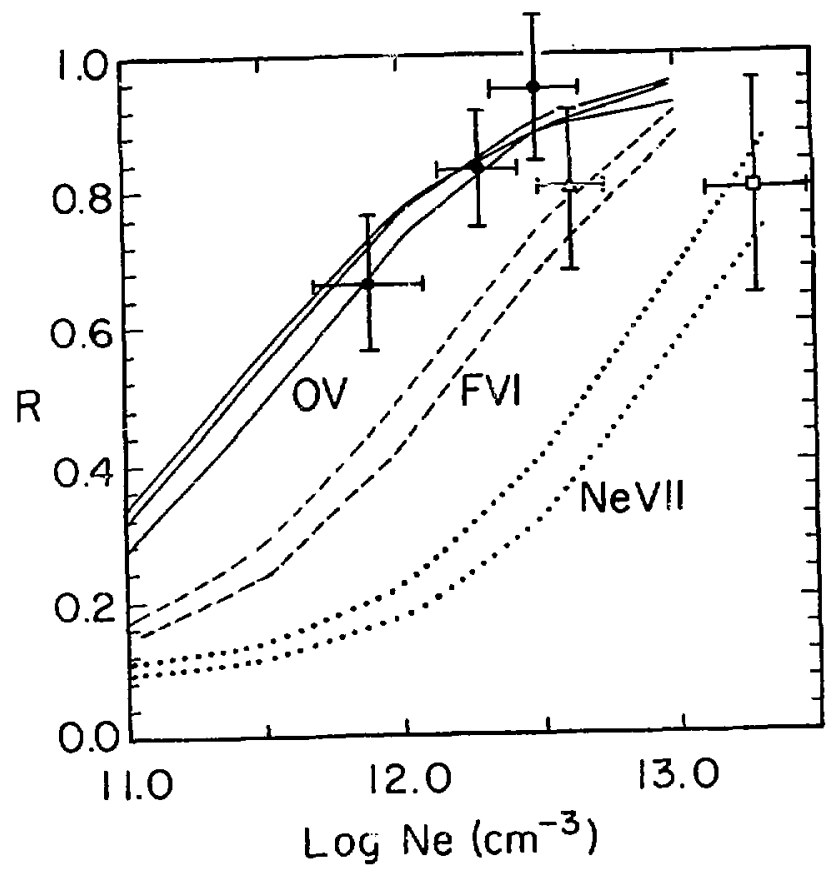

Fig. V.6 Comparison of experimental values and theoretical computations using the R-matrix method for three ions. a) O V -- solid circles (experiment); solid curves (theory) for three electron temperatures, $14 \mathrm{eV}$. $22 \mathrm{el}^{\prime \prime}$ and $34 \mathrm{eV}$. b) F Vl .. empty triangle (experiment); dashed curves (theory) for two temperatures, $22 \mathrm{eV}$ (upper curve) $43 \mathrm{eV}$ (lower curve). c) Ne VII -- empty square (experiment); dotted curves (theory) for two temperatures, $27 \mathrm{eV}$ (upper curve) and $54 \mathrm{eV}$ (lower curve). 


\section{Table V.2}

\section{Comparison Between Experiment and A-matrix Calculations}

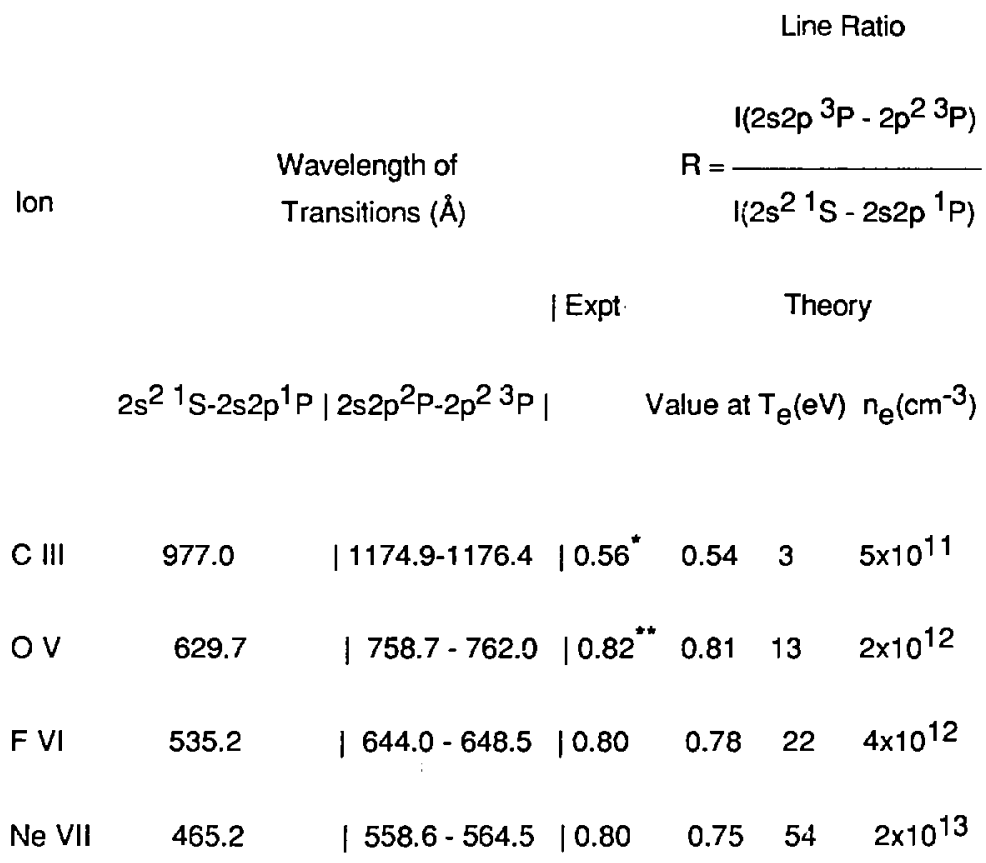

"Distorted wave method from Bhatia gave a value of $1.2 \mathrm{at}$ similar $T_{e}$ and $n_{e}$. "Distorted wave method from Bhatia gave a value of 0.9 at similar $T_{e}$ and $n_{e}$. 
emissions had high signal to noise ratios. We estimated the values of the ratio $R$ and obtained density values from the computed curves using the $R$-matrix method. We then compared the values obtained from the microwave interferometer and found that the density values obtained by these two totally different methods agree within $50 \%$. The results are summarized in Table V.3. Therefore, the electron density diagnostic from line ratios of the intrinsic impurities can serve as a rough and simple alternative to the traditional methods in the range $10^{10}$ to $3 \times 10^{12} \mathrm{~cm}^{-3}$. For higher $\mathrm{n}_{\mathrm{e}}$, other impurities like $\mathrm{F}$ or Ne must be introduced into the plasma and a better measurement of $\mathrm{T}_{e}$ is required if we want to use this method to measure the $n_{e}$ of the plasma. One of the applications of the line ratio is to obtain an estimate of the radial density profile. (For this method to work, the radial distribution of an impurity ion species must be highly localized as in the case of tokamak plasmas.) While it is true that microwave interferometers or beam attenuation detectors can be used to obtain radial profiles, it is very expensive to install many of them to obtain an appropriate profile. In some machines, the plasma edge is not well diagnosed. Under such circumstances, impurity line ratios would be very useful to obtain rough estimation on the parameters $T_{e}$ and $n_{e}$ near the edge. 
Table V.3

\section{Electron Density Diagnostic Using Line Ratio Method}

Ion Shot no.-date

Ratio R

$$
\begin{array}{cc}
n_{e} \text { from ratio } R & n_{e} \text { from TMX-U } \\
\left(\times 10^{12} \mathrm{~cm}^{-3}\right) & \text { density diagnostic }
\end{array}
$$

N IV $31-2 / 9 / 84$

0.7

0.5

$1^{* *}$

NIV $\quad 15-5 / 6 / 85$

0.74

1

1

ov $\quad 19-8 / 6 / 82$

0.88

4

3.9

OV

$22-1 / 25 / 83$

0.80

1.5

1.5

OV

$25-2 / 22 / 85$

0.90

4

2.5

\footnotetext{
"The physical quantity measured was the line density $\int n_{e} d l$. The electron density was obtained by assuming a reasonable radial profile.

"For this shot, there was a leak in the machine. The nitrogen came from the atmospheric air. We assumed that the NIV ions were near the edge of the plasma in this shot.
} 


\section{Chapter VI Conclusions}

In this paper we have discussed how spectroscopic methods are emploved to study the impurity properties in TMX-U and to develop possible $n_{e}$ and $T_{e}$ diagnostics. We found that the most abundant impurities in TMX-U are $\mathrm{C}, \mathrm{N}, \mathrm{O}$ and $\mathrm{Ti}$. $\mathrm{Si}$ is measured under some situations. From the time histories of the ion emissions, we conclude that large fractions of oxygen and nitrogen are associated with the neutral beams. From the measursd Doppler wicth of the broadened lines of $\mathrm{N} V$ emissions, we estimated that the kinetic energy of the ions was as high as $16 \mathrm{keV}$ when the neutral beams were turned on; this is additional evidence that the nitrogen is associated with neutral beams. We also found that the main source of carbon is the plasma wall. In general, the concentration of each of the impurities is low $(<1 \%)$, and the power radiated by them is less than $10 \mathrm{KW}$, which is a smali portion of the total input power to the plasma. The concentrations of the impurities carı be reduced substantially by glow discharge cleaning(GDC). The titanium gettering significantly lowers the impurity densities and neutral pressure. This effect persists for several shots after the gettering was stoppped. Without GDC and titanium gettering of a plasma shot, the carbon impurity in the plasma is so abundant that plasma startup is difficult, most probably because a significant amount of power has been radiated by the carbon ions. The experiment also showed that the vacuum wall of the TMX-U device can be cleaned up by the plasma shots. Also, ion cyclotron resonance frequency (ICRF) heating introduces impurities into the plasma and the abundance of these impurities increases with the ICRF power.

In adaition, the spectroscopic data shows that there was no significant accumulation of impurity ions in the thermal barrier region; this is consistent with an analytic model at the current barrier depth of $500 \mathrm{eV}$ and the average impurity charge state $Z$ of 4 .

Neutral beams were employed to inject nitrogen into the central cell to study impurity transport in TMX-U. We estimated the alobal confinement time of 
well as from the decay time histories from the ion emissions. The value was found to be between 1 and $10 \mathrm{~ms}$. We also studied the radial transport of impurities in TMX-U with a one dimensional diffusive/convective transport model. For the normal shots, we found that the values of the diffusive constant $D$ and convective velocity $V_{0}$ which best describe the data are close to those obtained in tokamak devices, but quite different from those obtained during the nitrogen-beam-injection experiment.

Experimental measurements of $\mathrm{Si}$ IIl line ratios obtained from TMX-U mirror plasmas, near the density $10^{11} \mathrm{~cm}^{-3}$ and at temperatures near the ionization equilibrium temperature, have confirmed the importance of the resonance structures in the collision strengths used in plasma diagnostics modeling. The new results significantly improve the reliability of estimates of the electron density and temperature of the solar corona and of flares based on Si IIl line intensity ratios. We also obtained spectra of the $\Delta n=0(2-2)$ transitions of Be I-like ions -- C III, O V, F VI and Ne VII, emitted from the TEXT tokamak. The electron temperature and densities in the region where the emissions originated were measured independently by non-spectroscopic methods. The line ratios were compared with different sets of computations performed by several authors. We found that the experimental data agrees quite well with the theoretical line ratio values obtained using the R-matrix technique, which includes the effects of resonances in the computation of the electron impact excitation rates. The results show that at temperatures close to ionization equilibrium, line ratios of the low- $Z$ Be sequence ions computed by the R-matrix method can be used as reliable electron density diagnostics. 


\section{Appendix A Details and Calibrations of the Normal Incidence Spectrograph}

This appendix is a supplement to Chapter II with a more detailed description of the 1024-channel normal incidence spectrograph and its calibration.

\section{A.1. Spectrograph}

An outline drawing of the spectrograph is shown in figure II.1 (in the main text). The spectrograph has seven gratings; each of them is $13 \times 13 \mathrm{~mm}$ in size with a radius of curvature of $0.4 \mathrm{~m}$. The optical system of the spectrograph is shown in figure A.1. The gratings are mounted in two rows along the Rowland circle, each row being either slightly above or below the Rowland circle plane. One of the seven gratings is selected by tilting (or rotating) the instrument about the entrance slit. The f/number of the system is defined by the preslit and the entrance slit. The aperture formed by the entrance slit and preslit ensures that only one grating at a time is illuminated. In general, we used an aperture which was about $f / 30$ wide by $f / 100$ high. In case of radial scan, we limiteo the aperture to about $1 / 100$ wide by $f / 100$ high which corresponds to a radial resolution of about $4 \mathrm{~cm}$ in the CC plasma of the TMX-U.

The detector is located opposite to the gratings. A $4 \times 40 \mathrm{~mm}$ photosensitive strip on the microchannel plate (MCP) ${ }^{155,156}$ is placed along the Rowland circle as shown in figure A.1. The EUV emissions are focused onto the detector after diffracting from the selected grating.

The characteristics of the seven gratings are shown in Table A.1. An overcoating of gold has been used for gratings with short wavelength spectral range while aluminum and magnesium flouride has been used for gratings with 


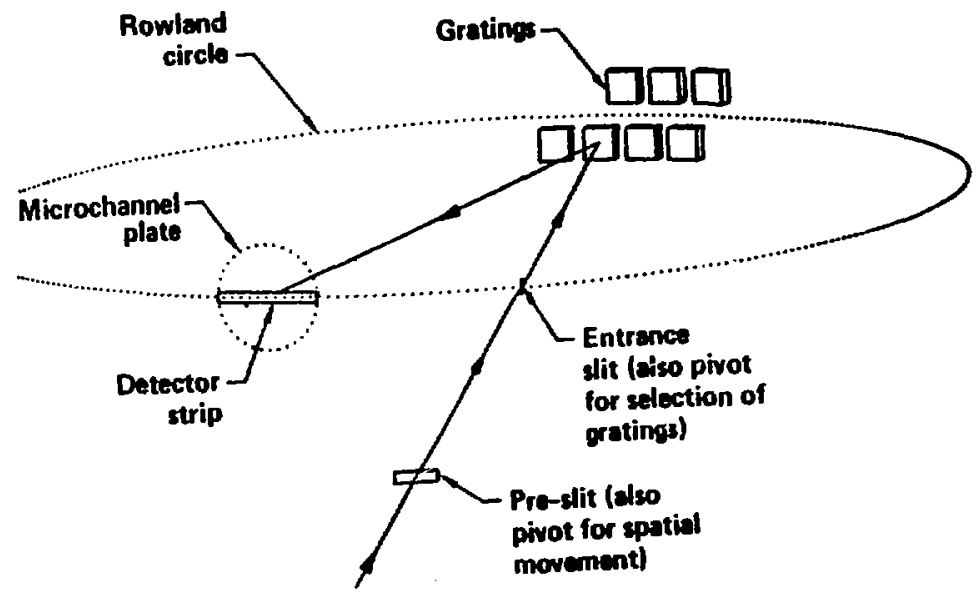

Fig. A.1 Grating orientation of the spectrograph 50 . 
Table A.1 Grating Characteristics

\begin{tabular}{lclll}
\hline $\begin{array}{l}\text { Groove } \\
\text { densuty } \\
(\mathrm{l} / \mathrm{mm})\end{array}$ & Blaze(Å) & Coating & $\begin{array}{l}\text { Spectral } \\
\text { range }(\AA)\end{array}$ & Resolution(Å) \\
\hline 600 & 750 & Au & $210-1845$ & $\sim 4.1$ \\
1200 & 2064 & Al, MgF 2 & $1370-2200$ & $\sim 2.1$ \\
3600 & 300 & Au & $290-560$ & $\sim 0.7$ \\
3600 & 300 & Au & $550-830$ & $\sim 0.7$ \\
3600 & 300 & Au & $810-1080$ & $\sim 0.7$ \\
2400 & 1000 & Al, MgF 2 & $1035-1460$ & $\sim 1.0$ \\
2400 & 1000 & Al, MgF 2 & $1450-1870$ & $\sim 1.0$ \\
& \multicolumn{5}{c}{} \\
\hline
\end{tabular}


longer wavelength spectral range to enhance the reflectivity of the gratings.

A magnesium fluoride filter (which cuts off emissions below $1200 \AA$ ) can be inserted to reduce second or higher order difrractions.

The spectrograph can be aligned along the optical axis with use of a telescope and an insertable mirror. The alignment can be done without opening the valve of the spectrograph thus maintaining the high vacuum during the aligning process.

\section{A.2. Detector System}

The detector system of the normal incidence spectrograph is presented in figure A.2. The detector is a windowless image intensifier tube coupled to a Reticon S-series 1024 pixel photodiode array 157,158. The image intensifier consists of a high-strip current microchannel plate(MCP) with a Cul photocathode. Behind the MCP, there is a fiber-optics bundle with P-20 phosphor deposited on its face. The fiber-optics bundle is coupled to the 1024-channel photodiode array.

As shown in figure A.2, when an EUV photon strikes the MCP Cul photocathode, a photoelectron is created and is acclerated down one of the channels by a bias voltage applied between the two surfaces of the MCP. When the electron collides with one of the channel walls, it knocks out several more electrons which in turn will be accelerated and will knock out more electrons. This results in an electron gain of $10^{2}-10^{4}$ when biac voltage of $500-1000 \mathrm{~V}$ is used. The bias voltage is adjustable so that a larger dynamic range of the instrument can be obtained.

The elect. ons emitteci at the other side of the MCP are accelerated and proximity focused onto the phosphor screen of the riber-optics bundle by a $5000 \mathrm{~V}$ potential difference. When the electrons strike the phosphor screen, visible photons are produced and are transmitted by the fiber-optics bundle to the photodiode array (the photodiode array consists of 1024 pixels; each pixel is 2.5 $\mathrm{mm}$ high with center spacing of $25 \mu \mathrm{m}$ and the length of the entire array is 25.6 $\mathrm{mm}$ ). The incident photons deplete a certain quantity of charge from each pixel of 


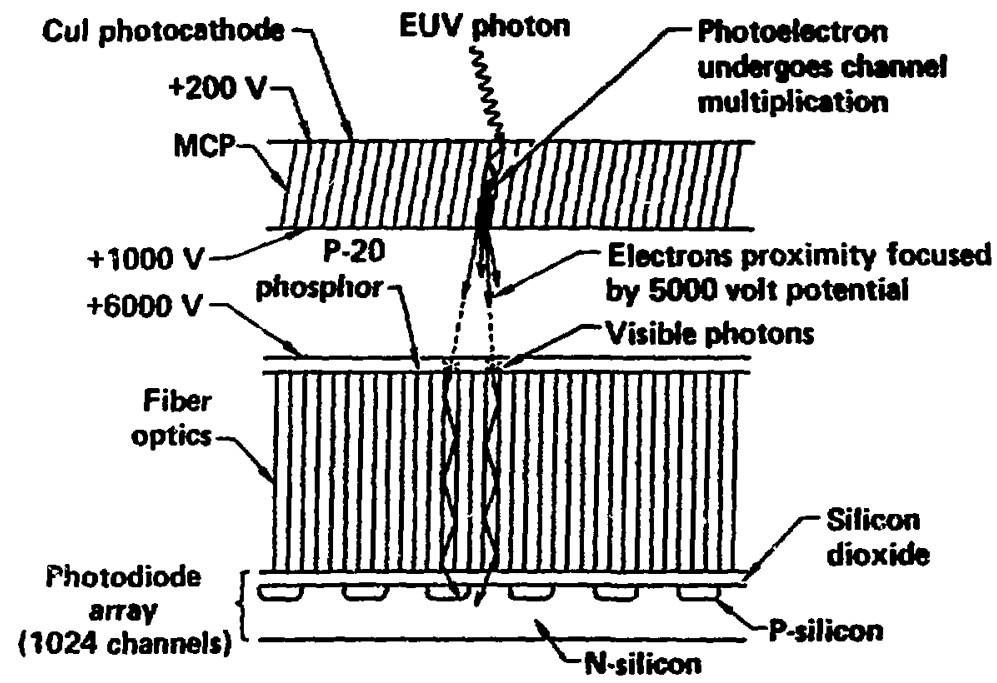

Fig. A.2 Intensified photodiode array of the normal incidence spectrograph 50 . 
the photodiode array. After some exposure time, scanning electronics recharge each pixel in sequence and output a voltage pulse for each pixel; the magnitude of the output voltage is proportional to the charge required tu restore the pixel to background level which in turn is proportional to the intensity of the incident light. The period between successive outputs of each pixel is the integration time. The time required to recharge the entire array is always the same, namely $4 \mathrm{~ms}$ (or $4 \mu \mathrm{s}$ for each pixel). However, the exposure time can be increased to obtain a higher output signal by programming the scanning circuit to wait for a certain time before recharging the array. The waiting time can be as long as $56 \mathrm{~ms}$ with a $4 \mathrm{~ms}$ inciement. Also, we can scan groups of pixels that we are interested using this clock rate and skip the other pixels with a clock rate which is 4 times faster. By this method, if we only select a few pixels, the integration time can be reduced to a value slightly larger than $1 \mathrm{~ms}$.

The voltages obtained are processed by the Reticon P. 10245 evaluation circuit board. (The circuit has been slightly modified so that the clock signals are supplied externally by a high precision crystal clock.) The evaluation circuit generates video signals which are available for direct measurement using a simple apparatus like an oscilloscope.

For the convenience of storing the data, we built additional controlling electronic circuits to process the video output. It is first digitized at a 12-bit level. This 12-bit data together with 4-bit status are read by a CAMAC memory. The CAMAC memory can then be read by a desktop computer or the TMX-U data acquisition system using a popular standard bus, GPIB. On the other hand, the electronic controller can be programmed by the destop computer with use of another bus called GPIO. The data acquisition system is shown in figure A.3.

We have developed software using the desktop computer to acquire data, reset the electronics, process the data, store it in floppy disks and print out the results for each shot. Once set up, the spectrograph can do all these automatically witiout human interaction or operation. 


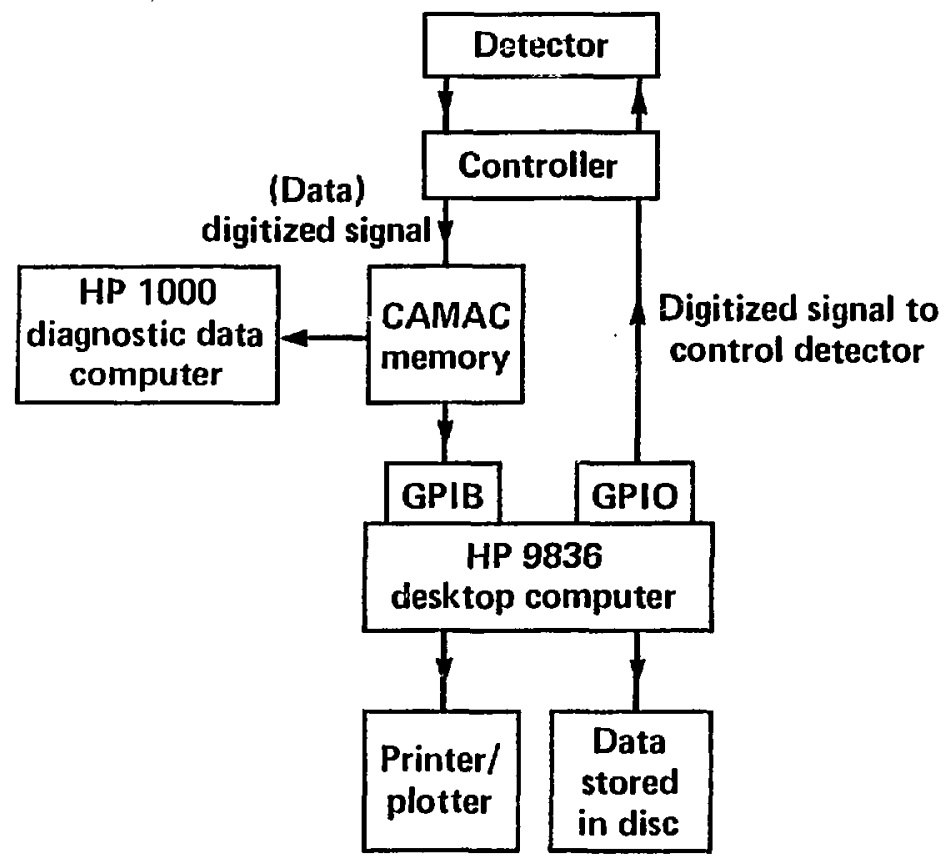

Fig. A.s Data acquisition of the spectrosoopic system. 


\section{A.3. Intensity Calibration}

We did the intensity calibration in two different ways. One calibration was performed using the Synchrotron Ultraviolet Radiation Facility (SURF) at the National Burea of Standards (NBS). The other calibration was done using the extreme ultraviolet calibration equipment (EUVCE) ${ }^{54}$ at The Johns Hopkins University. Throughout this project, only the absolute calibration of the survey grating $A$ was required. We therefore used this grating as an example to show how we calibrate the instrument. The calibration results obtained from these two methods are consistent with each other.

\section{A.3.a. Calibration at SURF II}

The electron storage ring of SURF II generates intense synchrotron radiation. The intensity and the spectral distribution of the radiation can be readily calculated ${ }^{159}$. Upon knowing the parameters of the synchrotron, the entrance slit size of the spectrograph and the distance between the slit and the center of the electron plane, the radiation flux for eacin pixel in the photodiode array can be determined.

The spectrograph is mounted on a movable table at NBS to allow easy alignment. Because the intensity of the radiation decreases sharply with increasing angle above or below the electron orbital plane, the spectrograph is carefully aligned such that the center of the entrance slit is brought as close to the plane of the electron orbit as possible, i.e. we try to align the optical axis of the spectrograph to a tangential line of the eleciron orbit.

Because of the continuous spectrum nature of the synchrotron radiation, we can do photometric calibration for each pixel of the detector However, this continuous nature also causes a serious problem in the calibration; the contribution of the flux due to second and higher orders of diffraction can be more 
significant than that of the first order diffraction. At SURF, the two electron energies available are $243 \mathrm{MeV}$ and $140 \mathrm{MeV}$. At $243 \mathrm{MeV}$, the flux peaks at $\sim 175 \AA$ And decreases rapidly for wavelength below $\sim 100 \AA$ and above $300 \AA$. . The wavelength of interest for grating $A$ is between $300 \AA$ to $1800 A$. Therefore, in this case, the higher order flux is more important than the flux due to first order diffraction. This can be seen in figure A.4 in which the spectral flux distribution for grating $A$ of different orders at 243-MeV electron energy are shown. At 140 $\mathrm{MeV}$, the flux peaks at $\sim 700 \AA$ (figure A.5). The contribution of the higher order flux are much less significant when compared to the case of $243 \mathrm{MeV}$. If contributions of orders larger than 2 can be neglected , we can simply "unfold" the first and second order contributions making use of measured signals at both $140 \mathrm{MeV}$ and $243 \mathrm{MeV}$ energies. Since we have two known sources of incident flux, it is easy to solve for the two unknowns, namely the first order efficiency and the second order efficiency ${ }^{160}$. However, unlike the case of the $140 \mathrm{MeV}$ energy, the contribution to the signals due to third and fourth order diffractions at $243 \mathrm{MeV}$ cannot be ignored. We therefore only use the $140 \mathrm{MeV}$ energy in calibrating grating $A$ and use a different approach to obtain the calibration.

First of all, as shown in Fig. A.5, at $140 \mathrm{MeV}$, the second order flux (i.e. flux arising from second order diffraction) is relatively small compared to the first order flux. Secondly, at a given pixel position, the second order efficiency is smaller than $(\leq 50 \%)$ the first order efficiency. Therefore, the detector signals ( flux $x$ efficiency) due to second order are much smaller than that due to first order at $140 \mathrm{MeV}$. To a first approximation, the second order contribution can be neglected. To obtain a better calibration, we could make use of the ratios of second order to first order signals of certain lines measured from mirror and tokamak discharges and from EUVCE measurements.

The second order diffsaction of a line at $500 \AA$ will show up at a pixel position corresponding to $1000 \AA$. For convenience of discussions, the wavelengths of the first order signal on the detector are taken as reference. Thus, the efficiency of a first order line at detector position $500 \AA$ is expressed as $e_{1}(500)$; the efficiency of a second order line at detector position $1000 \AA$ is expressed as 


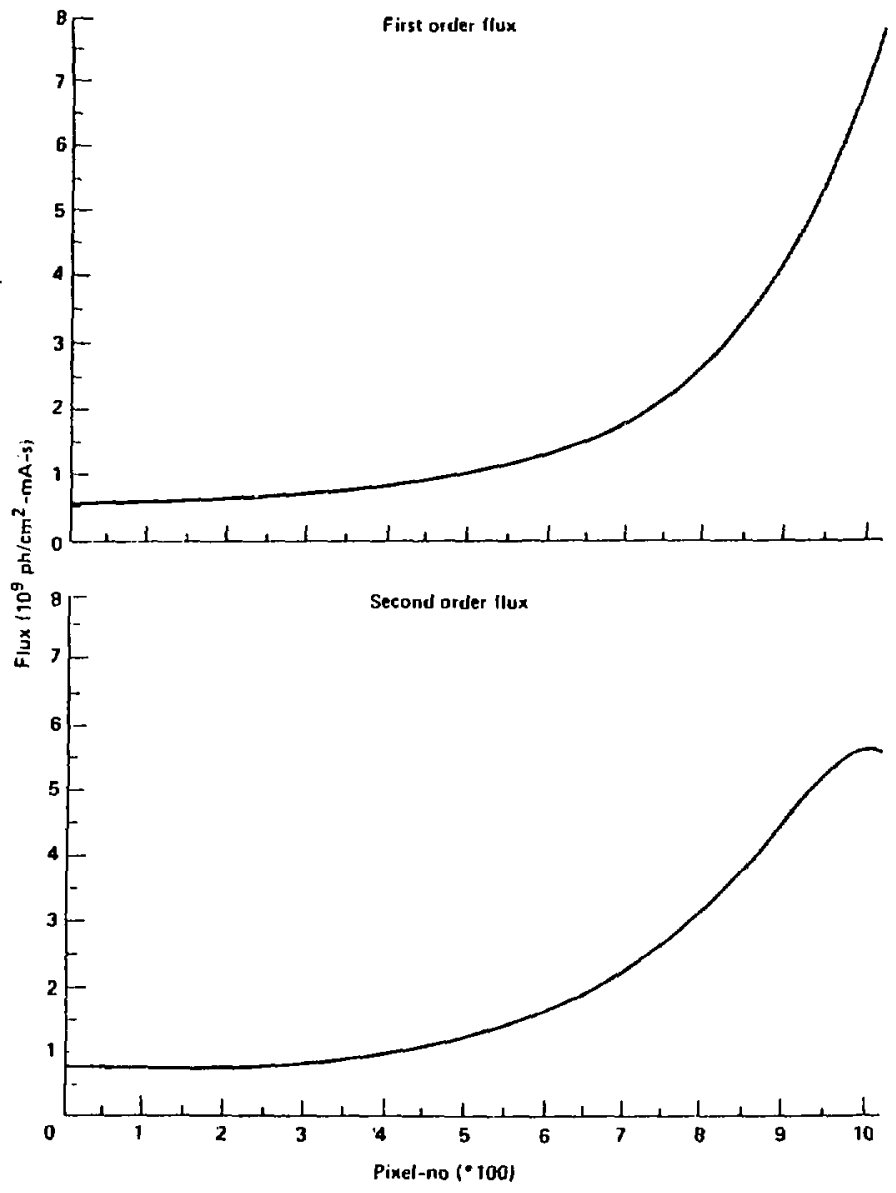

Fig. A.4 Distribution of first and second order flux of 243-Mev beam on detector. 


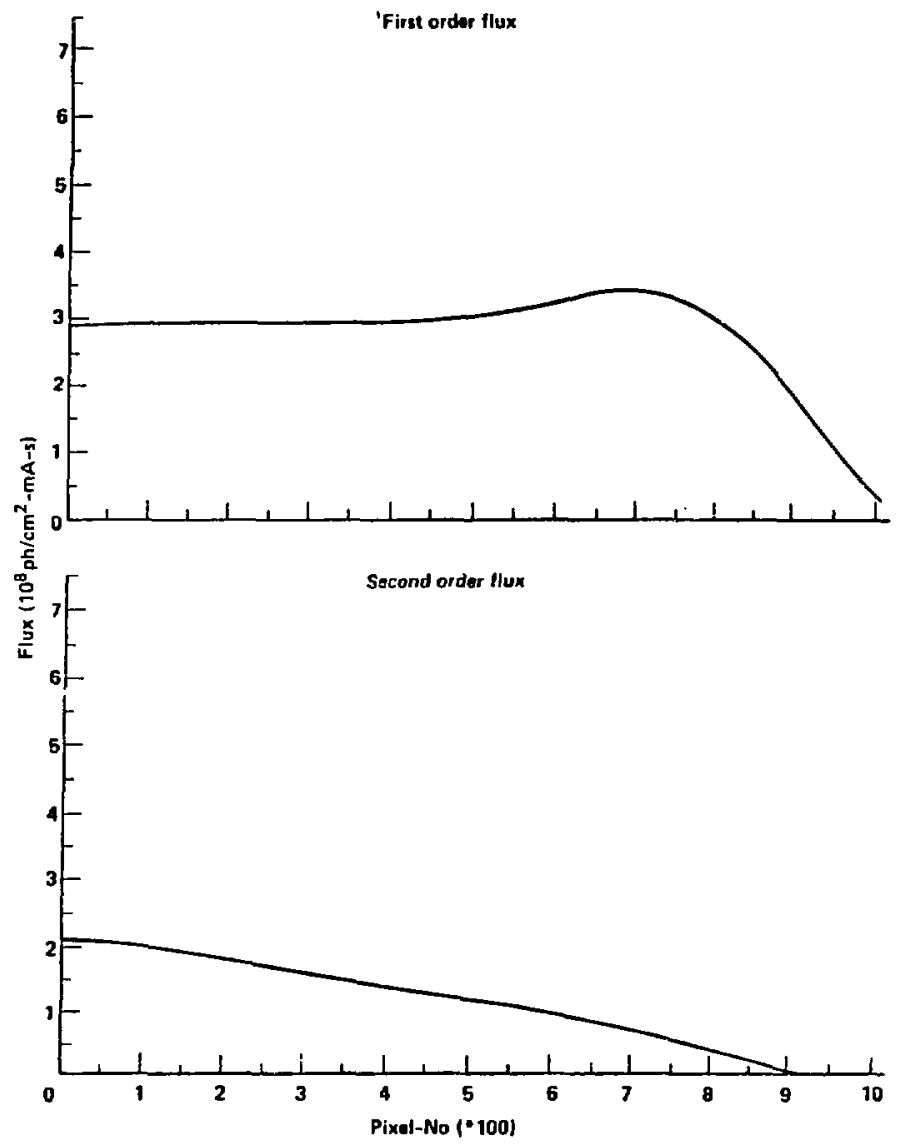

Fig. A.5 Distribution of first and second order flux of 740-Mev beam on detector. 
$e_{2}(1000)$ even though the real wavelength of the line is $500 \AA$. The ratio that we measure is $e_{2}(1000) / e_{1}(500)$. This kind of ratio can be obtained by comparing the counts of the two corresponding lines appear in the spectrum. If the lires are fairly strong, the accuracy can be as good as $10 \%$. We can extrapolate or interpolate the measured ratios to other detector positions.

According to the above convention, the measured signal $S$ arising fro.n synchrotron radiation at detector position $\lambda$ is given by

$$
S(\lambda)=F_{1}(\lambda) e_{1}(\lambda)+F_{2}(\lambda) \epsilon_{2}(\lambda)
$$

where $F_{1}(\lambda)$ and $F_{2}(\lambda)$ are the known first order and second order flux respectively at detector position $\lambda$. Equation (A.1) car also be expressed as

$$
S(\lambda)=e_{1}(\lambda)\left[F_{1}(\lambda)+F_{2}(\lambda)\left\{e_{2}(\lambda) / e_{1}(\lambda)\right\}\right]
$$

We can obtain $e_{1}(\lambda)$ values in first approximation by setting $\epsilon_{2}(\lambda)=0$ as in equation (A.1). The $e_{2}(\lambda)$ is then

$$
e_{2}(\lambda)=\left\{e_{2}(\lambda) / e_{1}(\lambda / 2)\right\} e_{1}(\lambda / 2)
$$

The $e_{1}(\lambda)$ and $e_{2}(\lambda)$ obtained in this way are an overestimate. A more reasonable $\Omega_{1}(\lambda)$ would be the average between this one(obtained in the latest calculation) and the one obtained in the previous step. We can cgain obtain another set of $e_{2}(\lambda)$ values ano repeat the process. The $e_{1}(\lambda)$ will converge rapidly because each time if we start computing with an overestimated $e_{1}(\lambda)$, 
the next-step $e_{1}(\lambda)$ will be underestimated and vice versa. The iterative process is repeated until the the difference between the $e_{1}(\lambda)$ values obtained in two subsequent steps is within $5 \%$ of its own value. We reached this criterion in three steps of computations. The final results are presented in figure A.6.

The effect due to the polarized nature of the synchrotron radiation was eliminated by calibrating the instrument at two perpendicular orientations and averaging the results.

There is also a problem of scattered light arising mainly from the imperfect ruling of the gratings. This effect is very difficult to eliminate. Judging from the line spectra obtained from mirror plasmas or EUVCE measurements we conclude that the uncertainty due to this effect in our synchrotron calibration is about $25 \%$ for grating $A$.

The overall uncertainty of our absolute calibration for grating $A$ is about $33 \%$. The sources of the errors and their contributions to the overall uncertainty are tabulated in Table A.2. (Here, we assume that the probability distributions of the uncertainties can be approximated by Gaussians. The \% uncertainty is defined as the ratio of the standard deviation to the measured value. That is, there is a seventy per cent chance that the actual value lies within the error bar.)

\section{A.3.b. Calibration Using EUV Calibration Equipment}

The extreme ultraviolet calibration equipment (EUVCE) was designed and constructed by the plasma group at Johns Hopkins University. The configuration of the EUVCE is shown in figure A.7. The EUVCE consists of a high power hollow cathode lamp ${ }^{161}$ which contains atomic and ionic species at a pressure between $10^{-4}$ to $10^{-5}$ torr; the pressure can be controlled by varying the amount of gas flowing through the lamp. Different gases are allowed to flow through the lamp so that a large range of different emission lines can be obtained. The spectral 


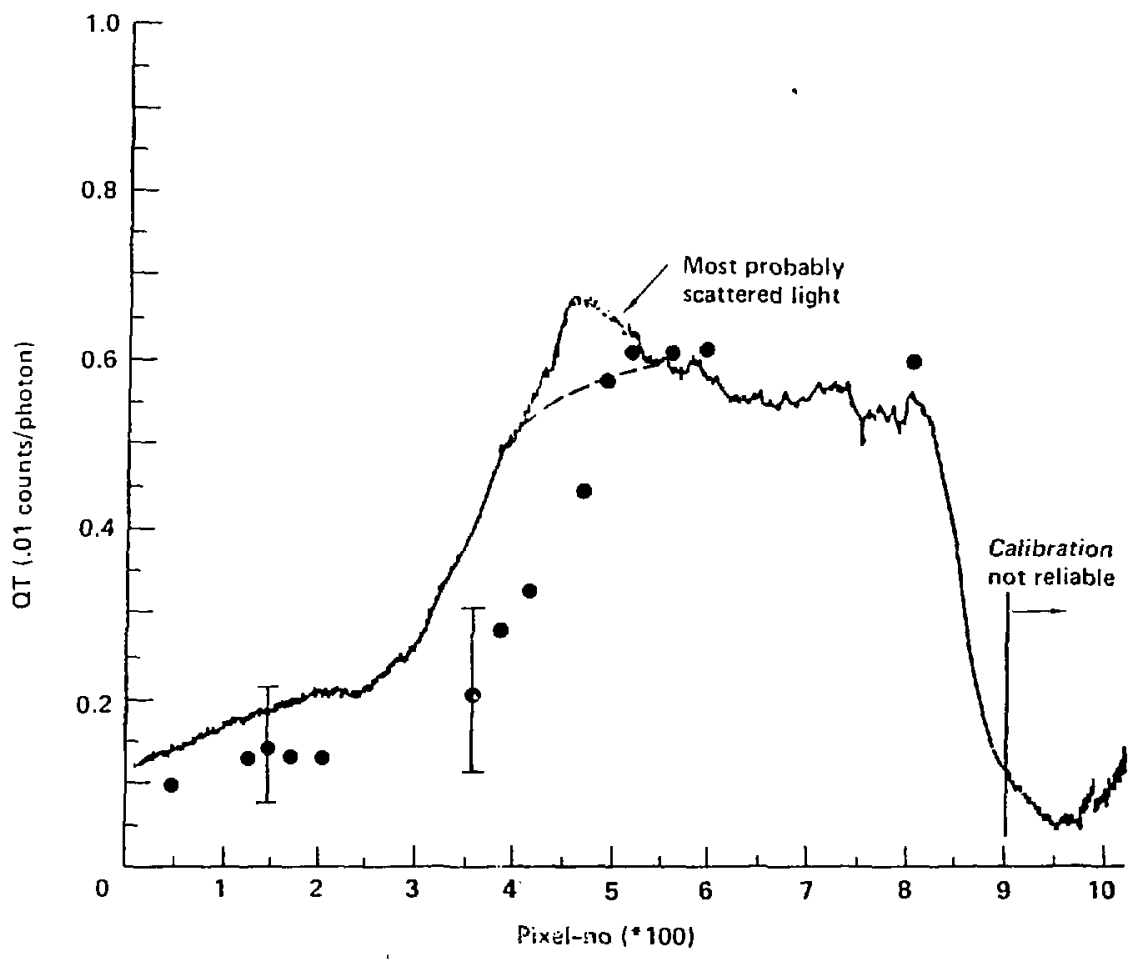

Fig. A.6 Photometric calibration from the SURF $\|$ at NBS (solid curve) and from EUVCE (solid dots). 
Table A.2 Uncertainties in the Calibration of Spectrograph (for Survey Grating)

a) SURF I| Calibration

Sources of Uncertainties

Scattered light

alignment

slit area

high orders diffraction

SURF II parameters

non-uniformity of grating

statistical noise

others
$\%$ Uncertainty ${ }^{+}$

25

10

10

10

10

5

5

5

b) EUVCE Calibration

alignment 30

reference detector calibration 20

reading of diode current 15

statistical noise $\quad 10$

fluctuation of light source $\quad 10$

others 5

overall

+see text for explaination 


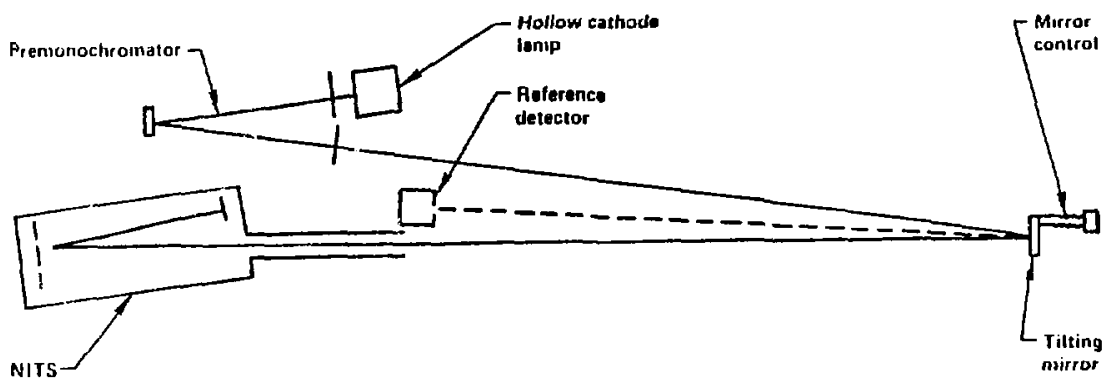

Fig. A.7 Calibration of the normal incidence time-resolving spectrograph (NITS) using the EUVCE. 
radiation emitted from the lamp will pass through a differentially pumped preslit and enters a $0.4 \mathrm{~m}$ monochromator which selects a desired spectral line. The selected spectral line is then reflected by a mirror which is overcoated with gold. The mirror can be tilted by two external micrometers so that the reflected beam can enter the reference detector or the instrument to be calibrated. In general, the reference detector is an NBS-calibrated photodiode or phototube. All the light monitored by the reference detector will be detected by the instrument detector.

The calibration of the instrument is done by comparing the signals measured by the already-calibrated reference detector and the instrument detector. For some weak spectral lines, the electric current produced in the reference diode is very small and we have to measure the charge accumulated in the diode over a certain period of time, say, two minutes. The corresponding current is equal to the measured charge divided by the integrated time. During the calibrating process, the photon source from the cathode lamp must be kept constant because a few minutes are required to switch the tilting mirror from the instrument-mode to the reference-mode and back. By observing the signals trom the lamp using the spectrograph, we found that the intensity of the photon source changed less than a few per cent over a period of ten minutes. Therefore, the error due to the drift in the cathode lamp is only a few per cent which is minor compared to other sources of error. The uncertainty in the calibration of the reference diode contributes about $20 \%$ error; the alignment may have $30 \%$. Also, the reading of the diode current, the noise in the spectrograph detector, scattered light from the monochromator, and dispersion of the spectral line on the spectrograph detector will contribute certain errcis. The overall uncertainty in the calibration of the spectro'jraph using EUVCE is about $43 \%$ (see Table A.2). Note that the uncertainties that we have discussed are those of absolute calibration. For relative calibration (i.e. relative sensitivity between different pixel positions), the uncertainties involved are much smaller. For example, in the relative calibration, misalignment of the instrument coes not affect the result and errors arising from , non-unifromity of the grating can be ignored. The results of the calibration are shown together with the results from SURF II in Fig. A.6. 


\section{APPENDIX Time Histories of Beam-injected Particles}

In chapter IV, we discussed the time histories of different ionization states in the plasma in the $\mathrm{N}$-beam injection experiment. Instead of solving the coupled-charged-state equations numerically, here we try to obtain approximate analytic expressions. In the experiments, the recombination and charge exchange processes of the impurities were always slower than either the ionization process or the confinement loss rate, so we will neglect the effects due to recombination and charge exchange throughout the discussions here. With this asssumption, we can express the time evolution of a given impurity charge-state in the form

$$
\frac{\partial n_{k}}{\partial t}=\langle o v\rangle_{\text {ion, } k-1} n_{e} n_{k-1}-\langle o v\rangle_{\text {ion, }} k^{n} n_{k}-\left(\frac{\partial n_{k}}{\partial t}\right)_{\text {conf }}
$$

where $n_{k}=$ density of the $k$-th ionization state of the injected element (nitrogen here)

$\langle o v\rangle_{\text {ion, }, k}=$ ionization coefficient out of the $k$-th state

$$
\mathrm{n}_{\mathbf{e}}=\text { electron density }
$$

$\left(\partial n_{k} \partial t\right)_{\text {conf }}=$ the rate of change in the $k$-th state due to confinement

Analytic expressions for the time histories of different ionization states can only be obtained by making additional simplifying assumptions: nonvarying plasma parameters during the time of injection, and cylindrical symmetry. 
i.e. $\quad<o v\rangle_{\text {ion }, k}=\left\langle o r>_{\text {ion }, k o} f_{\text {ion }, k}(r)\right.$

$$
\begin{array}{ll}
n_{e} & =n_{e o} e^{(r)} \\
n_{k} & =n_{k o}(t) f_{k}(r)
\end{array}
$$

In expression (B.2), the index "o" indicates the peak value of the corresponding quantity with radial profile $f(r)$. By substituting (B.2) into (B.1) and integrating over the whole volume of the plasma, we obtain

$$
\frac{d N_{k}(t)}{d t}=\frac{N_{k-1}(t)}{\tau_{\text {ion, } k-1}}-\frac{N_{k}(t)}{\tau_{\text {ion,k }}}-\frac{N_{k}(t)}{\tau_{c, k}}
$$

where

$$
\begin{aligned}
& N_{k}=\text { total number of particles of the k-th ionization state in the } \\
& \text { plasma } \\
& \tau_{c, k}=\text { the global confinement time of the } k \text {-th state } \\
& \tau_{\text {ion,k }}=\frac{1}{\gamma_{k}<\sigma v>_{\text {ion, } k o n_{e o}}} \\
& \gamma_{k}=-\int_{\text {ion, } k}{ }^{(r) f} e_{e}^{(r) r d r}-\sim 1 \\
& \int t_{e}(r) r d r \quad 2
\end{aligned}
$$

In equation (B.3), in the case of singly ionized ion, we have to include a source term and set the neutral density to be zero. The source term is just equal to the 
total number of beam injected particles attenuated in the plasma in a unit time. This was measured by the beam attenuation detector $(B A D)^{60}$.

$$
\text { i.e. } \quad \frac{d N_{1}}{d t}=S(t)-\frac{N_{1}}{\tau_{\text {ion, } 1}}-\frac{N_{1}}{\tau_{C, 1}}
$$

Actually, $S(t)=S_{0}=$ constant when the nitrogen neutral beams were turned on and $S(t)=0$ when the beams were truned off. Under these conditions, the solutions for (B.3) and (B.4) can easily be obtained by straight forward integration. On defining

$$
\frac{1}{\tau_{k}} \equiv \frac{1}{\tau_{\text {ion, } k}}+\frac{1}{\tau_{c, k}}
$$

the solution can be expressed in a recursive form as follow.

(a) When the nitrogen beams were turned on,

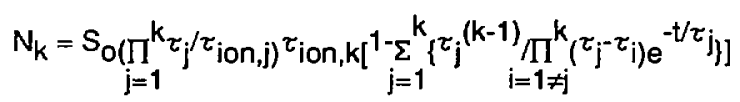

(b) When the nitrogen beams were turned off,

$$
N_{k}=\sum_{j=1}^{k} N_{k j} e^{-t / \tau_{k}}
$$


where

$$
\begin{aligned}
& N_{k k}=N_{k o}-\sum_{j=1}^{k-1 \geq 1} N_{k i} \\
& N_{k j}=\left(N_{k-1 j /} \tau_{k-1}\right)\left(\tau_{k} \tau_{j}\left(\tau_{j}-\tau_{k}\right) \quad(j \neq k)\right.
\end{aligned}
$$

and $\mathrm{N}_{\mathrm{ko}}$ are the number of particles $\mathrm{N}_{\mathrm{k}}$ at the instance when the beams were turned off.

From equation (B.7), we see that the decay of a state when the beams were turned off cannot be expressed in a simple exponent form $e^{-t / \tau}$. This is because the characteristic atomic physics times are comparable to the transport times. The decay rates of a particular ionization state also depends on the ciecay rates of the previous states. In equation (B.6) and (B.7), the only adjustable parameters are the $\tau_{c, k}$. By putting in different values of $\tau_{c, k}$, we can obtain different time profiles. For simplicity, we have assumed that all the giobal confinement times of different ionization states $\tau_{c, k}$ are the same and are equal to $\tau_{c}$. We adjusted $\tau_{c}$ to yield a match between experimental data and time profiles predicted by equations (B.6) and (B.7).

The actual physically measured quantity is the brightness of the emissions from impurity ions integrated along the line of sight of the instrument.

i.e. $B_{k} \sim \int<\sigma v e_{e x} e^{n_{k}} d l$ 
Equations (B.6) and (B.7) predict only the time evolution of the total number of particles of a certain ionization state in the plasma. However, as discussed before, if the shape of the impurity ion density profiles do not change drastically in time, the time histories of the brightness are essentially the same as the corresponding time histories of the total number of particles in the plasma. The time profiles in Fig. IV.4 and Fig. IV.5 in Chapter IV were produced by these equations. 


\section{APPENDIX C One Dimensional Numerical Model for Radial Profiles of Impurity lons}

Here we describe in detail the one dimensional transport cade discussed in section IV.3. In this code, we assume symmetry in all but the radial coordinate (cylindrical geometry). This enables us to express the time evolution of a given impurity charge state in space and time in the form

$$
\frac{\partial n_{q}}{\partial t}+\underset{r}{\frac{1}{\partial r} I_{q}}=I_{q-1} n_{q-1}-\left(I_{q}+R_{q}\right) n_{q}+R_{q+1} n_{q+i}-n_{q} / \tau_{q}+S_{q}
$$

where $\mathrm{n}_{\mathrm{q}}=$ density of the $\mathrm{q}$-th ionization state of a particular element $\mathrm{I}_{\mathrm{q}}=$ ionization rate out of the $\mathrm{q}$-th state (equals the product of electron derısity and the corresponding ionization coeffiecient)

$\mathrm{R}_{\mathrm{q}}=$ recombination rate to the $\mathrm{q}$-th state

$\mathrm{s}_{\mathrm{q}}=$ the source term

$\tau_{q}=$ a confinement time that takes into account parallel loss and scrape off process at the limiter

and $I_{q}$ is the particle flux density. This flux is taken to be of the form

$$
r_{q}=-D_{q}(r)\left(\partial n_{q} / \partial r\right)+V_{q}(r) n_{q}
$$


where $\mathrm{D}_{\mathrm{q}}(r)$ is the Darticle diffusion coeficient, and $\mathrm{V}_{\mathrm{q}}(\mathrm{r})$ is a corivective velocity. These $t_{a}$ nspurt coefficients may be functions of the ionic charge as well as space and time. Similarly, the $\mathrm{I}_{\mathrm{q}}, \mathrm{A}_{\mathrm{q}}, \Gamma_{\mathrm{q}}$ and $\mathrm{S}_{\mathrm{q}}$ are functions of charge state, radius and time. In general, expressions for $D_{q}(r)$ and $V_{q}(r)$ should be obtained from theoretical calculations. In fusion devices, anomalous transports which deviate largely from theoretical predictions have observed. Therefore, the code should be allowed for arbitrary values for the transport coefficients. The transport coefficients are adjusted to yield a match between the code results and experimental data. Here, we assume that

$$
I_{q}=-D\left(\partial n_{q} / \partial r\right)+(r / a) V_{o} n_{q}
$$

where $D$ is a constant and $a$ is the limiter radius. Expression (C.3) automatically satisfies the boundary condition $\Gamma_{q}(0)=0$ provided $\left(\partial n_{q} / \partial r\right)=0$ at $r=0$. We also assume that $\tau_{q}=$ constant. For simplicity, we have only considered the steady state case. (For general case, please refer to ref. 162)

i.e. $0=-\frac{1}{r} \frac{\partial r \Gamma_{q}}{\partial r}+I_{q-1} n_{q-1}-\left(I_{q}+R_{q}\right) n_{q}+R_{q+1} n_{q+1}+n_{q} / \tau_{/ /}+S_{q}$

To obtain numerical solutions to the set of couple equations in Eq. (C.4), we specify a radial mesh consisting of $N$ zones and consider finite differences in $r$. The charqe state dimension is already in a discrete form. In each radial zone 
$1 \leq \mathrm{j} \leq \mathrm{N}$, we have a $(Z+1) \times 1$ matrix $\left(n_{j}\right)$ containing the $Z+1$ charge state densities $\mathrm{n}_{\mathrm{q}}$. The set of coupled equations in $\mathrm{Eq}$. (C.4) can be transfromed into a finite difference scheme for numerical solution ${ }^{163}$. The finite differencing enables us to express the set of coupled equations in Eq.(C.4) in the form :

$$
\mathbf{X} \cdot \mu=\mathbf{S}
$$

In Eq. (C.5), $\mu$ is an $N \times 1$ matrix whose elements are the $(Z+1) \times 1$ matrices $\left(n_{j}\right)$, Correspondingly, $X$ is an $N \times N$ matrix whose elements are $(Z+1) \times(Z+1)$ matrices. The matrix is tridiagonal because the second order difference operator only connects a given radial zone to its adjacent zones. $\mathrm{S}$ is also $\mathrm{Nx} 1$ whose elements are the $(Z+1) \times 1$ matrices $\left(S_{j}\right)$ which contain the source term in Eq. (C.4).

$\left(x_{11}\right)\left(x_{12}\right)$

$\ldots \ldots\left(x_{i j-1}\right)\left(x_{i j}\right)\left(x_{i j-1}\right) \ldots$

$\left(X_{N N-1}\right)\left(x_{N N}\right) \quad\left(n_{N}\right)$ $\left(n_{1}\right)$

$\left(S_{1}\right)$

$$
\left(n_{j}\right)=\left(S_{j}\right)
$$

$\left(S_{N}\right)$

(C.6) 
Moreover, the matrices $\left(x_{i j}\right)$, and $\left(X_{j j \pm 1}\right)$ are either tridiagonal or diagonal because the atomic processes connect only ionization states which are adjacent to each other. The solution is thus easily obtained by elimination on $\left(n_{j}\right)$ by assuming solutions of the form

$$
\left(n_{j}\right)=\left(E_{j-1}\right)\left(n_{j-1}\right)+\left(F_{j-1}\right)
$$

When Eq. (C.7) is substituted into (C.5), we obtain

$$
\left(E_{j-1}\right)=-\left[\left(X_{j j}\right)+\left(X_{j j+1}\right)\left(E_{j}\right)\right]^{-1}\left[\left(S_{j}\right)-\left(X_{j j+1}\right)\left(F_{j}\right)\right]
$$

for $1<j \leq N$ and $\left(X_{N N+1}\right)=(0)$. We can immediately start evaluating $\left(E_{j-1}\right)$ and $\left(F_{j-1}\right)$ at $j=N$ and scan down to $\left(E_{1}\right)$ and $\left(F_{1}\right)$. Having obtained all values of $\left(E_{j-1}\right)$ and $\left(F_{j-1}\right)$, we obtain the successive values of $\left(n_{j}\right)$ from equation (C.7) by scanning upwards, from $\left(n_{1}\right)$ to $\left(n_{N}\right)$. The boundary conditions at $j=N$ define starting values $\left(E_{N-1}\right)$ and $\left(F_{N-1}\right)$, while the boundary conditions at $j=1$ specify the first matrix $\left(n_{1}\right)$. Specifically, in our case

$$
\begin{aligned}
& \left(E_{N-1}\right)=-\left(X_{N N-1}\right)^{-1}\left(X_{N N-1}\right) \\
& \left(F_{N-1}\right)=+\left(X_{N N}\right)\left(S_{N}\right)
\end{aligned}
$$


Also, from the consistency of the recursion relation (equation (C.7)) with the boundary conditions, we can express $\left(n_{1}\right)$ as follow.

$$
\left(n_{1}\right)=\left[\left(X_{11}\right)+\left(X_{12}\right)\left(E_{1}\right)\right]^{-1}\left[\left(S_{1}\right)-\left(X_{12}\right)-\left(X_{12}\right)\left(F_{1}\right)\right.
$$

The remaining matrices $\left(n_{j}\right)$ can now be determined from equation (C.7). The boundary conditions that we have chosen are

a) the flux at the center $(r=0)$ is zero,

$$
\text { i.e. } \quad \Gamma_{q}(0)=0
$$

and b) the density of all the ionization states besides the neutrals at the limiter radius are zero; the value of the neutral density has to be specified.

$$
\begin{array}{lll}
\text { i.e. } & \mathrm{n}_{\mathrm{q}}(\mathrm{a})=0 & \text { for ions } \\
& \mathrm{n}_{\mathrm{o}}(\mathrm{a})=\mathrm{n}_{\mathrm{O}} \mathrm{a} & \text { for neutrals }
\end{array}
$$

The non-zero density of the neutrals at $r=a$ corresponds to a source of neutral impurities due to reflux or a flux from the plasma wall.

In case of beam-injected impurities, the source for +1 ions is the attenuation of beam particles injected along a central chord. The amount of current attenuated in the $j$-th zone can be expressed as 


$$
\delta l_{j}=\operatorname{Ar}_{j} l_{j+1} \delta r N
$$

where $\mathrm{I}_{j+1}$ is the atomic beam current at the $(j+1)$-th zone; $A r_{j}$ is the attenuation rate of the current at the $j$-th zone; $V$ is the average velocity of the beam particles; and $\partial r$ is the radial step size. The beam current at the $j$-th zone is given by

$$
I_{j}=I_{j+1}-\delta I_{j}
$$

The current at the boundary $\left.\right|_{N+1}$ is just equal to the beam current from the neutral beams and is measured. We then can obtain all $\delta \mathrm{l}_{\mathrm{j}}$ and $\mathrm{l}_{\mathrm{j}}$ by start evaluating them at $j=N$. The source $S_{j}$ at the $j$-th zone is related to $\delta l_{j}$ by

$$
S_{j}=\delta l_{j} /\left(2 \prod r \delta r L\right)
$$

where $L$ is the length of the plasma. The attenuation rate in (14) is given by

$$
A r_{j}=n_{e}\left(r_{j}\right)\left(\langle\sigma v\rangle_{i o n}\left(r_{j}\right)+\langle\sigma v\rangle_{C x}\right)
$$


where $<\sigma v>_{\text {ion }}\left(r_{j}\right)$ is the ionization coefficient ${ }^{164}$ of the neutrals at the $j$-th zone; $\langle\sigma \mathrm{VV}\rangle_{\mathrm{CX}}$ is the charge exchange coefficient of the neutrals with the plasma ions. The values of $\langle\mathrm{OVV}\rangle_{\mathrm{CX}}$ are obtained using the methods and values given in references 165-167. Actually, the path of the beam particles is along a diameter of the plasma. Therefore, to obtain the total +1 source term in a zone, we need to scan (C.14) and (C. 15) from $\mathrm{j}=\mathrm{N}$ to 1 and then back from 1 to $\mathrm{N}$. In this way, we obtain a value for the source term for each zone during each scan. The total source for a zone is the sum of these two terms. The results are shown in Fig. IV.10 IV. 13 in Chapter IV. 


\section{References}

1. F.F. Chen, introduction to Plasma Physics, New York: Plenum, 1974

2. H. Motz, The Physics of Laser Fusion, Academic Press, 1979

3. A. Hasegawa, Rev. Laser Eng., 13, 53(1985)

4. A. Hasegawa, Bull. Am. Phy. Soc., 30, 214(1985)

5. H. Daido et al., Bull. Am. Phy. Soc., 30, 7E1(1985)

6. L.A. Artsimovich et al., Plasma Phys. \& Controlled Nucl. Fusion 2, 595(1966)

7. L.A. Artsimovich, Nucl. Fusion, 12, 215(1972)

8. S.S. Medley, Bull. Am. Phy. Soc., 30, 111(1985)

9. P.E. Stott, Bull. Am. Phy. Soc., 30, 112(1985)

10. M. Yoshikawa, Bull. Am. Phy. Soc., 30, 113(1985)

11. A. Ogata and T. Ando, Nucl. Fusion, 20, 1471(1980)

12. E.M. Blake, Nuclear News, 28, 77(1985)

13. J. Sheffield, Proc. IEEE, 69, 885(1981)

14. C.C. Baker, Proc. IEEE, 69, 917(1981)

15. H.P. Furth, Nucl. Fusion, 15, 487(1975)

16. L.M. Kovrizhnykh, Nucl. Fusion, 24, 851(1984)

17. P. Rutherford, Nucl. Fusion, 20, 1086(1980)

18. T.J. Dolan, Fusion Research, Pergamon Press, P. 273-310 (1982)

19. T.C. Simonen, Proc. IEEE, 69, 935(1981)

20. D.D. Ryutov, Nucl. Fusion, 20, 1068(1980)

21. R.W. Moir, Proc. IEEE, 69, 958(1981).

22. D.E. Baldwin, Rev. Mod. Phys., 49, 317(1977)

23. T.K. Fowler, Nucl. Fusion, 9, 3(1969)

24. V.S. Gott, M.S. loffe, and V.G. Telkovsky, Plasma Phys. \& Controlled Nucl. Fusion Research, 3, 1045(1962)

25. C.C. Damm et al., Plasma Phys. \& Controlled Nucl. Fusion Research, 2, 253(1969) 
26. A.W. Molvik et al., Phy. Rev. Lett., 32, 1107(1974)

27. W.C. Turier et al., Nucl. Fusion, 19, 1011(1979) -

28. J.G. Cordey et al., Plasma Phys. and Controlled Nucl. Fusion, 2, 100(1977)

29. M.S. loffe et al., Sov. Phy. Jetp, 40, 1064(1975)

30. B.I. Kanaev et al., Nucl. Fusion, 19, 347(1979)

31. G.I. Dimov, V.V. Zakaidokov, and M.E. Kishinevskii, Sov. J. Plasma Phy., 2, 326(1976)

32. T.K. Fowler and B.G. Logan, Commt. Plas. Phys. Contr. Fus., 2, 167(1977)

33. K. Yatsu et al., Phys. Rev. Lett., 43, 627(1979)

34. F.H. Corensgen et al., Phy. Rev. Lett., 44, 1132(1980)

35. R. Breun et al., Plasma Phys. \& Controlled Nucl. Fusion Research, 1, 105(1981)

36. N. Hershkowitz et al., Plasma Phys. \& Controlled Nucl. Fusion Research, 1, 553(1982)

37. D.E. Baldwin and B.G. Logan, Phy. Rev. Lett., 43, 1318(1979)

38. F.H. Coensgen, T.C. Simonen, A.K. Chargin, and B.G. Logan, 'TMX Upgrade Major Project Proposal", Lawrence Livermore Nat. Lab., LLL-Prop- 172 (1980)

39. V.P. Pastukihov, Nucl. Fusion, 4, 3(1973)

40. D.P. Chernin, and M.N. Rosenbluth, Nucl. Fusion, 18, 47(1978)

41. T.D. Rognlien, and T.A. Cutler, Nucl. Fusion, 20, 1002(1980)

42. R.H. Cohen, I.B. Bernstein, J.J. Downing, and G. Rowlands, Nucl. Fusion, 20, 1421(1980)

43. S.W. Stallard, Y. Matsuda, and W.M. Nevins, Nucl. Fusion, 23, 213(1983)

44. D.P. Grubb et al., Phys. Rev. Lett., 53, 783(1984)

45. E. Hinov, Princetong Plasma Physic, Laboratory, Report MATT-194, Princeton, $\mathrm{NJ}(1962)$

46. W. M. Stacey, Jr., Fusion, John Wiley \& Sons, Chapter 6, (1984)

47. R.V. Jensen, D.E. Post, W.H. Grasberger, C.B. Tarter, and W.A. Lokke, Nucl. Fusion, 17, 1187(1977)

48. S.L. Allen, T.L. Yu, and T.J. Nash, LLNL, UCID-20026, 1984 
49. T.J. Nash, S.L. Allen, D.D. Dietrich, and R. Fortner, Bull. Am. Phys. Soc., 27, 408(1982)

50. R.E. Bell, M. Finkenthal, and H.W. Moos, Rev. Sci. instrum., 52, 1806(1981)

51. R.K. Richards, H.W. Moos, and S.L. Allen, Rev. Sci. Instrum., 51, 1(19i30)

52. O.T. Strand, Ph. D. Thesis, The Johns Hopkins University (1982)

53. E.S. Warden and H.W. Moos, Appl. Opt., 16, 1902(1977)

54. H.W. Moos, K.I. Chen, J.L. Terry, and W.G. Fastie, Appl. Optics, 18, 1209(1979)

55. R.P. Drake and H.W. Moos, Nucl. Fusion, 19, 407(1979)

56. R.P. Drake and H.W. Moos, Nucl. Fusion, 20, 599(1980)

57. S.L. Allen, O. T. Strand, H.W. Moos, R.I. Fortner, F.J. Nash, and D.D.

Dietrich, Lawrence Livermore National Laboratory, UCID-18883(1981)

58. O.T. Strand, H.W. Moos, and S.L. Allen, Nucl. Fusion, 22, 657(198\%;

59. O.T. Strand, H.W. Moos, and S.L. Allen, Nucl. Fusion, 23, 1675(1983)

60. J.H. Foote, A.W. Molvik, and W.C. Turner, Rev. Sci. Instrum., 54, 928(1983)

61. R.K. Gocdman and T.D. Rognlein, "Initial Simultaneous Thomson Scattering Measurements in the TMX-U Tandem Mirror", Lawrence Liver. Nat. Lab., Livermore, CA, UCID-1943(1983)

62. S.L. Allen, and R.H. Munger, Bull. Am. Phy. Soc., 27, 4Q13(1982)

63. T.L. Yu, S.L. Allen and H.W. Moos, J. Vac. Sci. Tech. A,3, 1077(1985)

64. T.L. Yu, S.L. Allen, H.W. Moos, and R. Zimmerman, Bull. Am. Phy. Soc., 27, 4O-18(1982)

65. R. Kane et al., J. Vac. Technol., A3, 1081(1985)

66. J.E. Osher, D.P. Grubb, and P. Poulsen, Bull. Am. Phy. Soc., 28, 4R12(1983)

67. S.L. Allen, C.A. Clower, R.P. Drake, J. Vac. Sci. Tech.,A1, 916(1983)

68. S.L. Allen, T.L. Yu, J.H. Foote and W.L. Pickles, J. Vac. Sci. Tech., A3 (June, 1986) 
69. D.E. Post, R.V. Jensen, C.B. Tartev, et al., Atomic Data and Nuciear Table, 20, 404(1977)

70. A.W. Molvik et al., Bull. Am. Phy. Soc., 29, 1S17(1984)

71 T. Nash, Lawrence Livermore Nat. Lab., Livermore, CA (Private Communication, 1984)

72. W.L. Pickles, J. Vac. Sci. Tech. A, 3, 1171(1985)

73. J.H. Foote, G.W. Coutts, L.R. Pedrotti, et al., Rev. Sci. Instrum., 56, $1117(1985)$

74. W.L. Hsu et al., J. Vac. Sci. Tech. A, 2, 1222(1984)

75. R.S. Devoto, L.L. LoDestro, and A.A. Mirin, "Trapping Fate in Tandem Barriers ", submitted to Nucl. Fusion (April, 1986)

76. H. Griem, Plasma Spectroscopy, McGraw Hill Book Company, P.101 (1964)

77. D.W. Posener, Australian J. Phys., 12, 184(1959)

78. P.A. Jansson, Deconvolution With Application in Spectroscopy, Academic Press, INC., (1984)

79. W. Lotz, Ap. J. Suppl., 14, 207(1967)

80. S.L. Allen, D.L. Correll, D.N. Hill, and T.L. Yu, Bull. Am. Phys. Soc., 30, 3R5(1985)

81. D. H. Hill, Private Communication, LLNL(1986)

82. R.J. Fonck, and R.A. Hulse, Phys. Rev. Lett., 52, 530(1984)

83. W.K. Leung, Ph.D. Thesis, "Investigation of Impurity Transport in Texas Experimental Tokamak", University of Texas at Austin(1984)

84. S.L. Allen, H.W. Moos, E.S. Mamar, and J.E. Rice, Nucl. Fusion, 23, 303(1983)

85. W.D. Langer, Nucl. Fusion, 22, 751(1982)

86. R.J. Hawryluk, S. Suckewer, and S.P. Hirshman, Nucl. Fusion, 19, 607(1979)

87. TFR Group, Plasma Physics, 20, 207(1978)

88. S.A. Cohen, J.L. Cecchi, and E.S. Marmar, Phys. Rev. Lett., 35, 1507(1975) 
89. G. Gibson, W.C. Jordan, and E.J. Lauer, Phys. Fluids, 6, 116(1963)

90. R.H. Cohen, M.E. Rensink, T.A. Cutler, and A.A. Mirin, Nucl. Fusion, 18, 1129(1978)

91. D.D. Ryutov, and G.V. Stupakov, JETP Lett., 26, 186(1977)

92. D.D. Ryutov, and G.V. Stupakov, Sov. J. Plasma Phys. . 4, 278(1978)

93. D.D. Ryutov, and G.V. Stupakov, Sov. Phys. Dokl., 2, 412(1978)

94. R.H. Cohen, Nucl. Fusion, 19, 1579(1979)

95. A.A. Mirin, S.P. Auerbach, R.H. Cohen, J.M. Gilmore, L.D. Pearlstein, and M.E. Rensink, Nucl. Fusion, 23, 703(1983)

96. J.H. Foote, etal., Journal of Fusion Energy, 2, 283(1982)

97. A. Simon, Phys. Rev., 98, 317(1958)

98. R.H. Cohen, W.M. Nevins, and G.V. Stupakov, Nucl. Fusion, 22, 611(1982)

99. J.R. Myra, and P.J. Catto, Phys. Rev. Lett., 48. 620(1982)

100. J.R. Myra, and P.J. Catto, Phys. Fluids, 26, 1836(1983)

101. R.P. Drake etal., Phys. Fluids, 25, 2110(1982)

102. D.P. Grubb et al., Phys. Fluids, 26, 2003(1983)

1n3. E.B. Hooper, Jr., T.A. Casper, M.D. Flammer, D.P. Grubb, A.C. Hunt, and

W.C. Turner, Bull. Am. Phys. Soc., 28, 1116(1983)

104. E.B. Hooper, Jr., D.E. Baldwin, T.K. Fowier, F.J. Kane, and W.C. Turner, Phys. Fluids, 27, 2264(1984)

105. E.B. Hooper, Jr., D.L. Correll, R.H. Cohen, J.M. Gilmore, and D.P. Grubb,

Phys. Fluids, 28, 3609 (1986)

105. O.T. Strand, H.W. Moos, and S.L. A.len, Nucl. Fusion, 23, 1675(1983)

106. T.L. Yu, S.L. Allen, and H.W. Moos, Bull. Am. Phys. Soc., 29, 8S17 (1984)

107. T.L. Yu, S.L. Allen, H.W. Moos, and T.J. Nash, Bull. Am. Phys. Soc., 30, 3R25 (1985)

108. T.J. Orzechowski, M.R. Carter, and R.H. Munger, Rev. Sci. Instrum., 54, 1615(1983)

109. R.J. Kane, W.L. Hsu, R.G. Kerr, B.E. Hills, P. Poulsen, and S. Hibbs, J. Vac.

Sci. Technol. A3, 1081(1985)

110. J. Foote, Private Communication, LLNL (1984) 
111. T.C. Simonen etal., Phys. Rev. Lett., 50, 1668(1983)

112. H. Griem, Plasma Spectroscopy, McGraw Hill Cornpany, 176(1984)

113. S.L. Allen, Ph. D. Thesis, The Johns Hopkins University(1980)

114. S.L. Allen, Lawrence Livermore Nat. Lab., Private Communication(1984)

115. D.W. Blair, JQSRT, 14, 325(1974)

116. M. Okamoto, and T. Amano, J. Comput. Phy., 26, 80(1978)

117. R.A. Hulse, Nucl. Fusion, 3, 259(1983)

118. R.J. Fonck, M. Finkenthal, R.J. Goldston, D.L. Herndou, R.A. Hulse, R. Kaita, and D.D. Meyerhofer, Phys. Rev. Lett., 49, 737(1982)

119. J.D. Strachan etal., Nucl. Fusion, 22, 1145(1982)

120. E.S. Marmar, J.E. Rice, J.L. Terry, and F.H. Sequin, Nucl. Fusion, 22, 1567(1982)

121. TFR Group, Nucl. Fusion, 23, 559(1983)

122. F.H. Sequin, R. Petrasso, and E.S. Marmar, Phys. Rev. Lett., 51, 455(1983)

123. J.C. Moreno, and E.S. Marmar, Phys. Rev. A, 31, 3291(1985)

124. R.D. Benjamin, A. Nudelfuden, J.L. Terry, and H.W. Moos, Rev. Sci. Instr.

$56,870(1985)$

125. R.D. Benjamin, J.L. Terry, and H.W. Moos, Bull. Am. Phy. Soc., 30, 5Q19 (1985)

126. K. Sato et al., Bull. Am. Phy. Soc., 30, 7R47(1985)

127. U. Feldman et al., J. Appl. Phys., 56, 2512(1984)

128. D. Wroblewski, H.W. Moos, and W.L. Rowan, Bull. Am. Phy. Soc., 30, ᄀR22 (1985)

129. U. Feldman, Physica Scripta, 24, 681 (1981)

130. H.R. Griem, Spectral Line Broadening by Plasmas, Academic Press, (1974)

131. T.L. Yu, M. Finkenthal, and H.W. Moos, Ap. J., June, 1986

132. P.L. Dufton, A. Hibbert, A.E. Kingston and G.A. Doschek, Ap. J., 274, 420 (1983) 
133. K.A. Berrington, P.G. Burke, J.J. Chang, A.T. Chivers, W.D. Robb, and K.T. Taylor, J. Phys. B, 8, 149(1974)

134. K.A. Berrington, P.G. Burke, M. LeDournent, W.D. Robb, K.T. Taylor, and Vo Ky Lan, Comput. Phys. Commun., 14, 367(1978)

135. K.L. Baluja, P.G. Burke, and A.E. Kingston, J. Phys. B, 13, 829(1980)

136. K.L. Baluja, P.G. Burke, and A.E. Kingston, J. Phys. B, 14, 1333(1981)

137. P.G. Burke, and W.D. Robb, Adv. Atomic Molec. Phys., 11, 143(1975)

138. K.R. Nicolas, J.-D.F. Bartoe, G.E. Brueckner, and M.E. Van Hoosier, Ap. J., 233, 741(1979)

139. A.H. Gabriel, and C. Jordan, Case Stud. At. Phys., 2, 40(1972)

140. P.L. Dúton, A.K. Berrington, P.G. Burke, and A.E. Kingston, Astron. Astrophys., 62, 111(1978)

141. P.L. Dufton, J.G. Doyle, and A.E. Kingston, Astron. Astrophys., 78, 318 (1979)

142. M. Malinovsky, Astron. Astrophys., 43, 101(1975)

143. S.E. Vernazza, and E.M. Reeves, Ap. J. Supp. Sev., 37, 485(1978)

144. W.L. Hodge, T.L. Yu, and H.W. Moos, Bull. Am. Phys. Soc., 25, 999(1980) 145. W.L. Hodge, B.C. Stratton, and H.W. Moos, Rev. Sci. Instr., 55, 16(1984) 146. K.W. Gentle, et al., Plasma Physics and Contr. Fusion, 26, 1407(1984)

147. M. Finkenthal and T.L. Yu et al., submitted to Ap. J., 1986.

148. D.R. Terry, W. Rowan, W.C. Connolly, and W.K. Leung, Proc. 10th Symp on Fusion Energy, 14, 959(1983)

149. F.P. Keenan, D.A. Berringtokn, P.G. Burke, A.E. Kinsgston, and P.L. Dufton, Mon. Not. R. Astron. Soc., 207, 459(1984)

150. H. Nussbaumer, and P.J. Storey, Astron. Astrophys., 64, 139(1978)

151. J.G. Doyle, A.E. Kingston, and R.H.G. Reid, Astron. Astrophys., 90, $97(1980)$

152. J.G. Doyle, P.L. Dufton, F.P. Keenan, and A.E. Kingston, Sol. Phys., 89, 243(1983)

153. F.P. Keenan, A.E. Kingston, P.L. Dufton, J.G. Doyle, and K.G. Widing, Solar Phys., 94, 91(1984) 
154. M.J. Seaton, Mon. Not. R. Astron. Soc., 127, 191(1964)

155. J.L. Wiza, Nucl. Instrum. \& Methods, 162, 587(1979)

156. J. Adams and B.W. Manley, IEEE Trans. Nucl. Sci. NS-13, 88(1966)

157. Y. Talmi and R.W. Simpson, Appl. Opt., 19, 1401(1980)

158. R.W. Simpson, Rev. Sci. Instrum., 50, 730(1979)

159. D.H. Tomboulian and P.L. Hartman, Phys. Rev., 102, 1423(1956)

160. E.B. Saloman, Appl. Opt., 14, 1391(1975)

161. F. Paresce, S. Kumar, and C.S. Bowyer, Appl. Opt., 10, 1904(1971)

162. R.A. Hulse, Nucl. Tech./Fusion, 3, 259(1983)

163. D. Potter, Computational Physics, John Wiley \& Sons, New York (1973)

164. W. Lotz, Z. Physik, 206, 205(1967)

165. J.H. Foote, A.W. Molvik, and W.C. Turner, Rev. Sci. Instrum., 54, 928(1983)

166. R.A. Hulse, D.E. Post, and D.R. Mikkelson, J. Phys. B, 13, 3895(1980)

167. R.F. Stebbings, A.C.H. Smith, and H. Ehrhardt, J. Geophys. Research, 69 , 2349(1964) 


\section{VITA}

The author, Tong Lai Y $\backslash$, was born in Hong Kong on September 28, 1954. He grew up in a peasant family of a small village. He attended Pok Wai Primary School, Heung Yee Kok Secondary School and graduated from Queen Elizabeth high school in 1972 in Hong Kong. He received a B. Sc. degree fram the physics department of the Chinese University of Hong Kong in 1977, and became a high school teacher in the two years followed.

He started graduate work in physics at Johns Hopkins University in the fall of 1979. In the first two years, he was a teaching assistant in general physics and advance electromagnetic theory. After passing his comprehensive examination in the spring of 1981, he began full time research in spectroscopic instrumentation under the guidance of Professor H.W. Moos. While at Johns Hopkins University, he received an M.A. degree from the physics department in May, 1981 and an M.S.E. degree from the Electrical Engineering and Computer Science department in May, 1982. In the summer of 1982, he began to conduct spectroscopic experiments on the TMX-U mirror device at the Lawrence Livermore National Laboratory. He also conducted spectroscopic experiments in the TEXT tokamak at the University of Texas at Austin during the fall of 1983 . This dissertation is the product of these two endeavors. 